\title{
Integrated Transmission and Distribution Control
}

K Kalsi

FK Tuffner

W Zhang

AR Fisher

ML Hauer
JC Fuller

$J$ Lian

LD Marinovici

FC Chassin

January 2013

Pacific Northwest

NATIONAL LABORATORY

Proudly Operated by Battelle Since 1965 


\title{
DISCLAIMER
}

This report was prepared as an account of work sponsored by an agency of the United States Government. Neither the United States Government nor any agency thereof, nor Battelle Memorial Institute, nor any of their employees, makes any warranty, express or implied, or assumes any legal liability or responsibility for the accuracy, completeness, or usefulness of any information, apparatus, product, or process disclosed, or represents that its use would not infringe privately owned rights. Reference herein to any specific commercial product, process, or service by trade name, trademark, manufacturer, or otherwise does not necessarily constitute or imply its endorsement, recommendation, or favoring by the United States Government or any agency thereof, or Battelle Memorial Institute. The views and opinions of authors expressed herein do not necessarily state or reflect those of the United States Government or any agency thereof.

\author{
PACIFIC NORTHWEST NATIONAL LABORATORY \\ operated by \\ BATTELLE \\ for the

\section{UNITED STATES DEPARTMENT OF ENERGY} \\ under Contract DE-AC05-76RL01830
}

Printed in the United States of America

Available to DOE and DOE contractors from the Office of Scientific and Technical Information,

P.O. Box 62, Oak Ridge, TN 37831-0062; ph: (865) 576-8401 fax: (865) 576-5728

email: reports@adonis.osti.gov

Available to the public from the National Technical Information Service, U.S. Department of Commerce, 5285 Port Royal Rd., Springfield, VA 22161

ph: (800) 553-6847 fax: (703) 605-6900

email: orders@ ntis.fedworld.gov online ordering:

http://www.ntis.gov/ordering.htm

This document was printed on recycled paper.

$(1 / 2013)$ 


\section{Integrated Transmission and Distribution Control}

$\begin{array}{ll}\text { K Kalsi } & \text { JC Fuller } \\ \text { FK Tuffner } & \text { J Lian } \\ \text { W Zhang } & \text { LD Marinovici } \\ \text { AR Fisher } & \text { FC Chassin } \\ \text { ML Hauer } & \end{array}$

January 2013

Prepared for

the U.S. Department of Energy

under Contract DE-AC05-76RL01830

Pacific Northwest National Laboratory

Richland, Washington 99352 


\section{Executive Summary}

Distributed, generation, demand response, distributed storage, smart appliances, electric vehicles and renewable energy resources are expected to play a key part in the transformation of the American power system. Control, coordination and compensation of these smart grid assets are inherently interlinked. Advanced control strategies to warrant large-scale penetration of distributed smart grid assets do not currently exist. While many of the smart grid technologies proposed involve assets being deployed at the distribution level, most of the significant benefits accrue at the transmission level. The development of advanced smart grid simulation tools, such as GridLAB-D, has led to a dramatic improvement in the models of smart grid assets available for design and evaluation of smart grid technology. However, one of the main challenges to quantifying the benefits of smart grid assets at the transmission level is the lack of tools and framework for integrating transmission and distribution technologies into a single simulation environment. Furthermore, given the size and complexity of the distribution system, it is crucial to be able to represent the behavior of distributed smart grid assets using reduced-order controllable models and to analyze their impacts on the bulk power system in terms of stability and reliability.

The objectives of the project were to:

- Develop a simulation environment for integrating transmission and distribution control,

- Construct reduced-order controllable models for smart grid assets at the distribution level,

- Design and validate closed-loop control strategies for distributed smart grid assets, and

- Demonstrate impact of integrating thousands of smart grid assets under closed-loop control demand response strategies on the transmission system.

More specifically, GridLAB-D, a distribution system tool, and PowerWorld, a transmission planning tool, are integrated into a single simulation environment. The integrated environment allows the load flow interactions between the bulk power system and end-use loads to be explicitly modeled. Power system interactions are modeled down to time intervals as short as 1-second.

Another practical issue is that the size and complexity of typical distribution systems makes direct integration with transmission models computationally intractable. Hence, the focus of the next main task is to develop reduced-order controllable models for some of the smart grid assets. In particular, HVAC units, which are a type of Thermostatically Controlled Loads (TCLs), are considered. The reduced-order modeling approach can be extended to other smart grid assets, like water heaters, PVs and PHEVs. Closed-loop control strategies are designed for a population of HVAC units under realistic conditions. The proposed load controller is fully responsive and achieves the control objective without sacrificing the end-use performance. Finally, using the T\&D simulation platform, the benefits to the bulk power system are demonstrated by controlling smart grid assets under different demand response closed-loop control strategies. 


\section{Acknowledgments}

The authors are grateful for the comments and feedback provided by David Chassin and Robert Pratt at the Pacific Northwest National Laboratory. The PNNL team would also like to thank the PowerWorld team, in particular, Tom Overbye, Mark Laufenberg, and Kollin Patten for their support in the software integration task. 


\section{Acronyms and Abbreviations}

$\begin{array}{ll}\text { COM } & \text { Component Object Model } \\ \text { DSM } & \text { Demand Side Management } \\ \text { DC } & \text { Direct Current } \\ \text { DLC } & \text { Direct Load Control } \\ \text { ETP } & \text { Equivalent Thermal Parameter } \\ \text { GUI } & \text { Graphical User Interface } \\ \text { HVAC } & \text { Heating, Ventilation, and Air Conditioning } \\ \text { ODE } & \text { Ordinary Differential Equation } \\ \text { PDE } & \text { Partial Differential Equation } \\ \text { PJM } & \text { Pennsylvania-New Jersey-Maryland Interconnection } \\ \text { PV } & \text { Photovoltaic } \\ \text { PHEV } & \text { Plug-In Hybrid Electric Vehicles } \\ \text { RTO } & \text { Regional Transmission Organization } \\ \text { TCL } & \text { Thermostatically Controlled Loads } \\ \text { T\&D } & \text { Transmission and Distribution }\end{array}$




\section{Contents}

Executive Summary $\quad$ iv

Acknowledgments $\quad \mathrm{v}$

Acronyms and Abbreviations $\quad$ vi

Contents vii

1.0 Introduction $\quad 1.1$

2.0 Reduced-order modeling and control of smart grid assets 2.1

2.1 Thermal dynamics of thermostatically controlled loads 2.1

$\begin{array}{ll}2.1 .1 & \text { Mass temperature dynamics } \\ 2.2 & 2.2\end{array}$

$\begin{array}{ll}\text { 2.1.2 Effect of heterogeneity } & 2.3\end{array}$

2.2 Design of reduced-order model for a population of HVAC units 2.4

2.2.1 Homogeneous aggregate model 2.4

$\begin{array}{lll}\text { 2.2.2 Heterogeneous aggregate model } & 2.7\end{array}$

2.3 Closed-loop controller design for HVAC unit aggregations 2.7

2.4 Simulation results $\quad 2.11$

2.4.1 Performance of aggregated model $\quad 2.11$

$\begin{array}{ll}\text { 2.4.2 Performance of aggregated control strategies } & 2.17\end{array}$

$\begin{array}{ll}2.5 \text { Conclusions } & 2.19\end{array}$

3.0 Integrated transmission and distribution simulation platform 3.1

3.1 Background 3.1

3.2 Software $\quad 3.2$

3.2.1 GridLAB-D $\quad 3.2$

3.2.2 PowerWorld Simulator $\quad 3.2$

3.3 Interface 3.3

$\begin{array}{ll}3.4 \text { Validation } & 3.4\end{array}$

4.0 Integrated T\&D Control Use Case $\quad 4.1$

5.0 Conclusions and Future Work $\quad 5.1$

References $\quad 5.2$

Appendix A Extensive Simulation Validation Results $\quad$ A.1 


\section{Figures}

Figure 1 The Equivalent Thermal Parameter (ETP) model of home heating/cooling system ............2.1

Figure 2 Coupled air and mass temperature dynamics ..................................................................2.2

Figure 3 Aggregate response for homogeneous and heterogeneous TCLs ....................................2.3

Figure 4 Illustration of 2D population flow, where the solid arrows represent the actual path the population moves, while the dashed arrows represent the corresponding approximated flow paths

Figure 5 Illustration of 2D population flows and transitions between "On" and "Off" states...........2.7

Figure 6 Air and mass temperature trajectories with setpoint $=75$, deadband $=1 \ldots \ldots \ldots \ldots \ldots \ldots \ldots \ldots . . .2 .8$

Figure 7 Illustration of density evolution with consideration of the lockout effect ........................2.10

Figure 8 Aggregated response with setpoint change from 75 to 76 degrees..................................2.12

Figure 9 Aggregated response with setpoint change from 75 to 70 degrees................................2.13

Figure 10 Aggregated responses with setpoint change from 75 to 74 degrees ..............................2.13

Figure 11 Aggregate response under sequential setpoint changes. (a) Setpoint change from 74 to 75 after the population reaches the steady state, (b) Sequence of setpoints applied before the population reaches the steady state

Figure 12 Aggregate responses with different number of discretization bins under a setpoint change from $75^{\circ} \mathrm{F}$ to $74^{\circ} \mathrm{F}$ (with 8 clusters)

Figure 13 Aggregated responses with different number of clusters under a setpoint change from $75^{\circ} \mathrm{F}$ to $74^{\circ} \mathrm{F}$ (\# bin $=60$ )

Figure 14 Aggregated model response including lock-out effect ................................................ 2.17

Figure 15 Direct load control for 30 minutes

Figure 16 Performance of tracking the dynamic regulation test signal; the real output refers to the aggregated power output of 5000 HVAC units, each of which has a 5-minute lock-out time ..2.19

Figure 17 Rough structure for PowerWorld Simulator and GridLAB-D interface ...............................

Figure 18 PowerWorld Simulator and GridLAB-D Validation Model - Magenta area represents the varying interface point

Figure 19 Time series plot of GridLAB-D and PowerWorld load values - Note PowerWorld represents a single-phase equivalent of the three-phase system ................................................

Figure 20 Modified PowerWorld model of three-node, two-area transmission system ....................4.1

Figure 21 Modified PowerWorld model of three-node, two-area transmission system with Bus 2 generator tripped and Branch 1-2 overloaded.

Figure 22 Modified PowerWorld model of three-node, two-area transmission system with Bus 2 generator tripped and Branch 1-2 congestion relieved by DLC-enabled HVACs at Bus 2........4.3

Figure 23 Time-series results for the total current flow through Branch 1-2 ...................................4.3

Figure 24 Outdoor temperature of $70^{\circ} \mathrm{F}$ and average cooling setpoint of $75^{\circ} \mathrm{F}$................................

Figure 25 Outdoor temperature of $85^{\circ} \mathrm{F}$ and average cooling setpoint of $75^{\circ} \mathrm{F}$............................... 3

Figure 26 Outdoor temperature of $100^{\circ} \mathrm{F}$ and average cooling setpoint of $75^{\circ} \mathrm{F}$...........................4

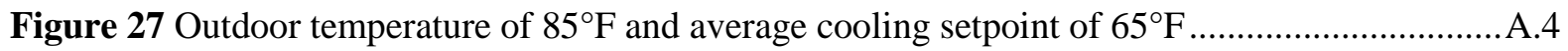


Figure 28 Outdoor temperature of $85^{\circ} \mathrm{F}$ and average cooling setpoint of $75^{\circ} \mathrm{F}$ using an alternate building design parameter distribution (e.g., different $U_{a}, H_{m}$, etc.).......................................

Figure 29 Outdoor temperature of $85^{\circ} \mathrm{F}$ and average cooling setpoint of $75^{\circ} \mathrm{F}$, applying a $+5^{\circ} \mathrm{F}$ delta for six hours to the cooling setpoint, then returning to normal .........................................6

Figure 30 Outdoor temperature of $85^{\circ} \mathrm{F}$ and average cooling setpoint of $75^{\circ} \mathrm{F}$, applying a $-5^{\circ} \mathrm{F}$ delta for six hours to the cooling setpoint, then returning to normal ........................................6

Figure 31 Outdoor temperature of $85^{\circ} \mathrm{F}$ and average cooling setpoint of $75^{\circ} \mathrm{F}$, applying a $+5^{\circ} \mathrm{F}$ delta for 2 hours to the cooling setpoint, then returning to normal, note that this includes a transition from one transient state to another without returning to steady state

Figure 32 Outdoor temperature of $85^{\circ} \mathrm{F}$ and average cooling setpoint of $75^{\circ} \mathrm{F}$, applying a $-5^{\circ} \mathrm{F}$ delta for 2 hours to the cooling setpoint, then returning to normal, note that this includes a transition from one transient state to another without returning to steady state

Figure 33 Outdoor temperature of $85^{\circ} \mathrm{F}$ and average cooling setpoint of $75^{\circ} \mathrm{F}$, applying a $+1^{\circ} \mathrm{F}$ delta for 10 minutes to the cooling setpoint, then returning to normal

Figure 34 Outdoor temperature of $85^{\circ} \mathrm{F}$ and average cooling setpoint of $75^{\circ} \mathrm{F}$, applying a $-1^{\circ} \mathrm{F}$ delta for 10 minutes to the cooling setpoint, then returning to normal

Figure 35 Outdoor temperature of $85^{\circ} \mathrm{F}$ and average cooling setpoint of $75^{\circ} \mathrm{F}$, applying a $+5^{\circ} \mathrm{F}$ delta for $t$ hours to the cooling setpoint, then returning to normal, using GridLAB-D thermostat lockouts

Figure 36 Outdoor temperature of $85^{\circ} \mathrm{F}$ and average cooling setpoint of $75^{\circ} \mathrm{F}$, applying a $-5^{\circ} \mathrm{F}$ delta for 2 hours to the cooling setpoint, then returning to normal, using GridLAB-D thermostat lockouts

Figure 37 Outdoor temperature of $85^{\circ} \mathrm{F}$ and average cooling setpoint of $75^{\circ} \mathrm{F}$, applying a $+1^{\circ} \mathrm{F}$ delta for 10 minutes to the cooling setpoint, then returning to normal, using GridLAB-D thermostat lockouts

Figure 38 Outdoor temperature of $85^{\circ} \mathrm{F}$ and average cooling setpoint of $75^{\circ} \mathrm{F}$, applying a $-1^{\circ} \mathrm{F}$ delta for 10 minutes to the cooling setpoint, then returning to normal, using GridLAB-D thermostat lockouts

Figure 39 Outdoor temperature of $85^{\circ} \mathrm{F}$, lockout times set to (near) zero, and a $25 \%$ reduction in load that releases back to normal operation after 30 minutes

Figure 40 Outdoor temperature of $100^{\circ} \mathrm{F}$, lockout times set to (near) zero, and a $25 \%$ reduction in load that releases back to normal operation after 30 minutes .

Figure 41 Outdoor temperature of $85^{\circ} \mathrm{F}$, lockout times set to (near) zero, and a $50 \%$ reduction in load that releases back to normal operation after 5 minutes

Figure 42 Outdoor temperature of $100^{\circ} \mathrm{F}$, lockout times set to (near) zero, and a $50 \%$ reduction in load that releases back to normal operation after 5 minutes

Figure 43 Outdoor temperature of $85^{\circ} \mathrm{F}$, lockout times set to two minutes, and a $50 \%$ reduction in load that releases back to normal operation after 5 minutes.

Figure 44 Outdoor temperature of $100^{\circ} \mathrm{F}$, lockout times set to two minutes, and a $50 \%$ reduction in load that releases back to normal operation after 5 minutes.

Figure 45 Outdoor temperature of $85^{\circ} \mathrm{F}$, lockout times set to (near) zero, and a $75 \%$ reduction in load that releases back to normal operation after 30 seconds

Figure 46 Outdoor temperature of $100^{\circ} \mathrm{F}$, lockout times set to (near) zero, and a $75 \%$ reduction in load that releases back to normal operation after 30 seconds 
Figure 47 Outdoor temperature of $85^{\circ} \mathrm{F}$, lockout times set to two minutes, and a $75 \%$ reduction in load that releases back to normal operation after 30 seconds

Figure 48 Outdoor temperature of $100^{\circ} \mathrm{F}$, lockout times set to two minutes, and a $75 \%$ reduction in load that releases back to normal operation after 30 seconds

Figure 49 Outdoor temperature of $85^{\circ} \mathrm{F}$ and average setpoint of $75^{\circ} \mathrm{F}$, lockout times set (near) zero

Figure 50 Outdoor temperature of $85^{\circ} \mathrm{F}$ and average setpoint of $75^{\circ} \mathrm{F}$, lockout times set to two minutes

Figure 51 Outdoor temperature of $85^{\circ} \mathrm{F}$, lockout times set to two minutes, a "lossless" system, with a $25 \%$ increase in load for 10 minutes, returning to normal for 50 minutes, then a $35 \%$ decrease in load for 10 minutes, and then returning to normal.

Figure 52 Outdoor temperature of $85^{\circ} \mathrm{F}$, lockout times set to two minutes, a "lossy" system, with a $35 \%$ increase in load for 10 minutes, returning to normal for 50 minutes, then a $25 \%$ decrease in load for 10 minutes, and then returning to normal.

Figure 53 Outdoor temperature of $100^{\circ} \mathrm{F}$, lockout times set to two minutes, a "lossless" system, with a $25 \%$ increase in load for 10 minutes, returning to normal for 50 minutes, then a $35 \%$ decrease in load for 10 minutes, and then returning to normal.

Figure 54 Outdoor temperature of $85^{\circ} \mathrm{F}$, lockout times set to two minutes, a "lossy" system, with a control signal that is a sinusoidal signal of power updated every minute with a maximum swing of $25 \%$ of normal power demand with a frequency of 1 hour

Figure 55 Outdoor temperature of $85^{\circ} \mathrm{F}$, lockout times set to two minutes, a "lossy" system, with a control signal that is a sinusoidal signal of power updated every minute with a maximum swing of $25 \%$ of normal power demand with a frequency of 15 minutes 


\subsection{Introduction}

Demand-side management (DSM) is considered to be a promising way to resolve many emerging challenges of the power system. Traditional DSM studies mostly focus on peak shaving and load shifting that take place at a relatively slow time scale. With the development of smart grid concepts and communication techniques, the real-time control of a large population of electric loads has received considerable attention. Among all electric loads, Thermostatically Controlled Loads (TCLs) account for a large fraction of electric demand. Heating, Ventilating, and Air Conditioning (HVAC) systems and water heaters are examples of TCLs. They use local hysteresis control to maintain either air or water temperature within a prescribed band around the temperature setpoint. Since TCLs are suited to shortterm service interruptions due to their intrinsic mass, they are good candidates to provide grid services. Modeling the behavior of populations of appliances under demand response is especially important to evaluate the effectiveness of the above mentioned programs and their proposed control strategies. Accurate aggregated behavior of demand response is needed to account for interactions of demand response with components of a large interconnected system, such as the eastern or western interconnections of the United States. The models of demand response should capture the dynamic behavior of the population, especially when demand response is expected to provide intra-hour services (ancillary services).

Dynamic modeling of thermostatic controlled loads was first studied in [1] and [2]. In [1], aggregate load models are designed to study the effects of cold load pickup after a service interruption. Functional models of devices, which account for factors such as weather and human behavior, are developed in [2]. A model of a large number of similar devices is then obtained through statistical aggregation of the individual component models. In [3], aggregated dynamic models are developed for a homogeneous population of thermostatic loads using stochastic diffusion models based on solving Fokker-Planck partial differential equations (PDEs). The approach is further extended in [4], where a closed-form solution of the coupled Fokker-Planck equations is derived and the need for having a heterogeneous population is motivated. The main contribution of [6] is to develop a finite dimensional aggregated state space model for air conditioning loads based on solving bilinear PDEs model. It is shown in [6] that an order of 200 is needed for the aggregated state space to accurately capture the detailed model dynamics. In [7] a statistical model based on Markov Chains is used to represent heterogeneity in a population of TCLs. The dynamics of solid mass temperature (second-order) are neglected in both detailed and aggregated representation. Also, the uncontrolled aggregated model is not accurate when compared with detailed simulations. In addition, an aggregated state transition based controllable model for a homogeneous population of HVACs and water heaters using detailed physical models is developed in [8]. In all the aforementioned approaches, the aggregated models are developed for steady state conditions; i.e., when there is no demand response or time varying changes in population and system configuration (weather conditions, solar gains, heat conductance etc.). Furthermore, the models ignore heterogeneity in the TCL population, which is extremely important to capture all the dynamics and thereby design effective control strategies.

The approach proposed in [8] is extended in [9] to include simple demand response strategies (thermostat setback) for HVAC units and the results are validated against GridLAB-D ${ }^{\mathrm{TM}}$. A commonly observed phenomenon associated with demand response programs is often called the "rebound" effect or an immediate spike in power demand when the thermostat setback is released back to normal operation. This is due to the fact that the control signal in the population model synchronizes all of the loads, 
resulting in a loss of load diversification, which takes some time to be re-established. Although, the aggregate model proposed in [9] is able to capture the peak of the re-bound effect, it is not able to capture the oscillations set up by the setpoint change due to the assumption made that diversity is always maintained.

The focus of this work is to develop aggregated controllable models for a heterogeneous population of TCLs that are able to capture demand response and time-varying effects of the system. The developed models include second-order dynamics due to the thermal mass of the house, which is ignored in most of the previous works. Accounting for this second-order effect is important to accurately capture the population dynamics, especially while applying demand response controls that rely upon the intrinsic thermal mass of the system to limit customer discomfort. A clustering strategy is developed to systematically deal with heterogeneity. The proposed aggregate model is able to accurately capture both steady state and the transient dynamics ( $>1$ second dynamics), which typically cannot be achieved by other aggregated models. The approach presented herein offers an adjustable trade-off between the complexity of the aggregated model and the ability of the model to accurately capture these dynamics. This feature makes the model suitable for various demand response applications that require different levels of modeling accuracy and complexity. With more complexity, the developed model can accurately estimate all of the transients caused by demand response signals, providing a valuable tool for stability analysis of the system under different demand response strategies. With reduced complexity, the developed model can still capture the main trend of the transients and maintain accuracy of steady state or average behavior, making it more amenable to design feedback control strategies for the population of devices to provide ancillary services.

In practice, a certain amount of time delay between successive "Off" and "On" cycles is required to prevent short cycling of a HVAC system. One of the contributions of this work is the development of an aggregate model that incorporates the time delay (or the so-called "lockout") effect of individual HVAC systems. Although this work focused on developing reduced-order models for HVAC units, the approach can be easily extended to develop aggregated models for other end-use loads (like water-heaters, dryers, refrigerators etc.), energy storage devices (battery and flywheels) and plug-in hybrid electric vehicles.

After developing aggregated controllable models, closed-loop control strategies are designed for a population of HVAC units under realistic conditions. The proposed load controller is fully responsive and achieves the control objective without sacrificing end-use performance. The proposed aggregated modeling and control strategies are validated through realistic simulations using GridLAB-D. The simulation results indicate that the proposed approach can effectively manage a large number of HVAC systems to provide various demand response services, such as frequency regulation and peak load reduction.

The effects of demand response and controllable distributed resources are not limited to the distribution system, but can also provide significant benefits at the transmission-level. However, the modern power system is typically divided into two levels of analysis: transmission and distribution. Transmission-level analysis focuses on bulk power transmission across significant distances. Distributionlevel analysis typically focuses on individual feeder sets or load centers of the system. Traditionally, these two levels of analysis are handled in two different pieces of software and are not coordinated or integrated. This limits the ability of planners and regulators to effectively assess the benefits associated with the use of distributed resources. 
For example, once a control strategy is developed for HVAC systems at the distribution level, its impacts on the transmission system need to be evaluated. Additionally, it may be useful (and more profitable) for distributed resource owners to participate for local control (e.g., voltage control) and widearea control (e.g., congestion management). To help examine the transmission-level impacts of the developed control strategies, as well as validate the aggregate model, the ability for transmission-level and distribution-level systems to interact is necessary. To aid in the validation process, this project developed an interface between a transmission-level solver (PowerWorld Simulator) and a distributionlevel analysis tool (GridLAB-D). This interface allows the examination and evaluation of distributionlevel device controls on the larger power system. 


\subsection{Reduced-order modeling and control of smart grid assets}

This section focuses on the design of aggregated models for HVAC units and on designing closedloop control strategies for these units under demand response programs. In Section 2.1, a description of the underlying physical model of thermostatically controlled loads is given. Also, the second-order effects of thermal mass and the effect of heterogeneity on the transient response of a population of TCLs are discussed. A second-order aggregate model for both homogeneous and heterogeneous populations is given in Section 2.2. The aggregate model results are compared to a more detailed, agent-based simulator. Results are discussed throughout each section and in more detail in Appendix A.

\subsection{Thermal dynamics of thermostatically controlled loads}

This section discusses the physical model of an individual load device. This individual device model forms the basis to developing an aggregated load model representing the behavior of a population of devices. Residential electric end-use loads can be divided into two principal classes: one for all nonthermostatic loads, such as lights and plugs; and one for all thermostatic loads, such as water heating and air conditioning. Thermostatic loads at the level of a single device give rise to pulse sequences where the pulse width and frequency arise from the control hysteresis and the heat balance in the load, while the power drawn changes between two or more relatively fixed values.

The Equivalent Thermal Parameter (ETP) model of the home heating/cooling system [1], representing such individual device loads, is shown in Figure 1.

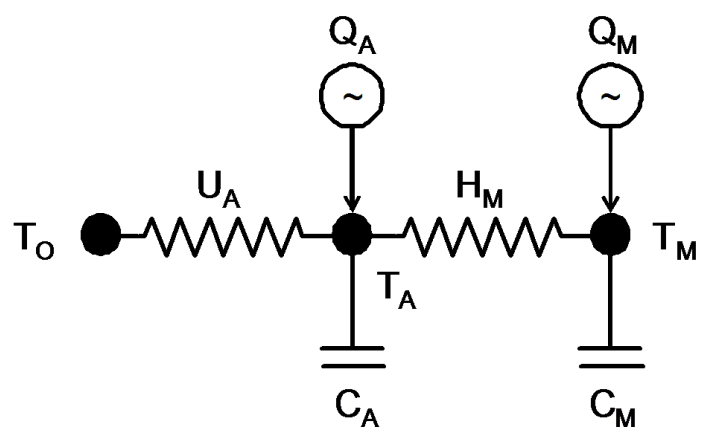

Figure 1 The Equivalent Thermal Parameter (ETP) model of home heating/cooling system

The formulation of the ETP model is given as follows:

$$
\begin{aligned}
& \frac{d T_{a}}{d t}=\frac{1}{C_{a}}\left[T_{m} H_{m}-T_{a}\left(U_{a}+H_{m}\right)+Q_{a}+T_{o} U_{a}\right] \\
& \frac{d T_{m}}{d t}=\frac{1}{C_{m}}\left[H_{m}\left(T_{a}-T_{m}\right)+Q_{m}\right]
\end{aligned}
$$

where,

$U_{a}$ is the conductance of the building envelope

$T_{o}$ is the outdoor air temperature 
$T_{a}$ is the indoor air temperature

$T_{m}$ is the inner mass temperature

$H_{m}$ is the conductance between the inner air and inner solid mass

$Q_{a}$ is the heat flux into the interior air mass

$C_{a}$ is the thermal mass of the air

$C_{m}$ is the thermal mass of the building materials and furnishings

$Q_{m}$ is the heat flux to the interior solid mass

The total heat flux $Q_{a}$ consists of three main contributing factors $Q_{i}, Q_{s}$, and $Q_{h}$ which are the heat gain from the internal load, the solar heat gain and the heat gain from the heating/cooling system respectively. Depending on the power state of the unit, the heat flux $Q_{a}$ could take the following two values:

$$
Q_{a}^{o n}=Q_{i}+Q_{s}+Q_{h}, \text { and } Q_{a}^{o f f}=Q_{i}+Q_{s}
$$

In the ETP model, $T_{a}$ and $T_{m}$ are the state variables, whose time course evolution depends on the model parameters that are collected as a parameter vector $\theta$ as follows:

$$
\theta=\left[U_{a}, C_{a}, H_{m}, C_{m}, Q_{i}, Q_{s}, Q_{h}, T_{o}\right]^{T}
$$

\subsubsection{Mass temperature dynamics}

Most existing aggregate modeling approaches for TCLs are based on first-order dynamics that neglect the second-order dynamics caused by the mass temperature $T_{m}$. Such simplifications often lead to an unsatisfactory prediction of the transient response caused due to, for example, a setpoint change. Figure 2 shows the evolution of the air temperature $T_{a}$ and mass temperature $T_{m}$ of a HVAC unit subject to a setpoint change from $74^{\circ} \mathrm{F}$ to $75^{\circ} \mathrm{F}$ at time $t=1 \mathrm{hr}$. It can be seen that it takes a much longer time for $T_{a}$ to increase from $74^{\circ} \mathrm{F}$ to $76^{\circ} \mathrm{F}$ during the time period $\left[t_{1}, t_{2}\right]$ than during the period $\left[t_{3}, t_{4}\right]$. This is due to the lower mass temperature $T_{m}$ during $\left[t_{l}, t_{2}\right]$, which significantly slows down the increase of the air temperature.

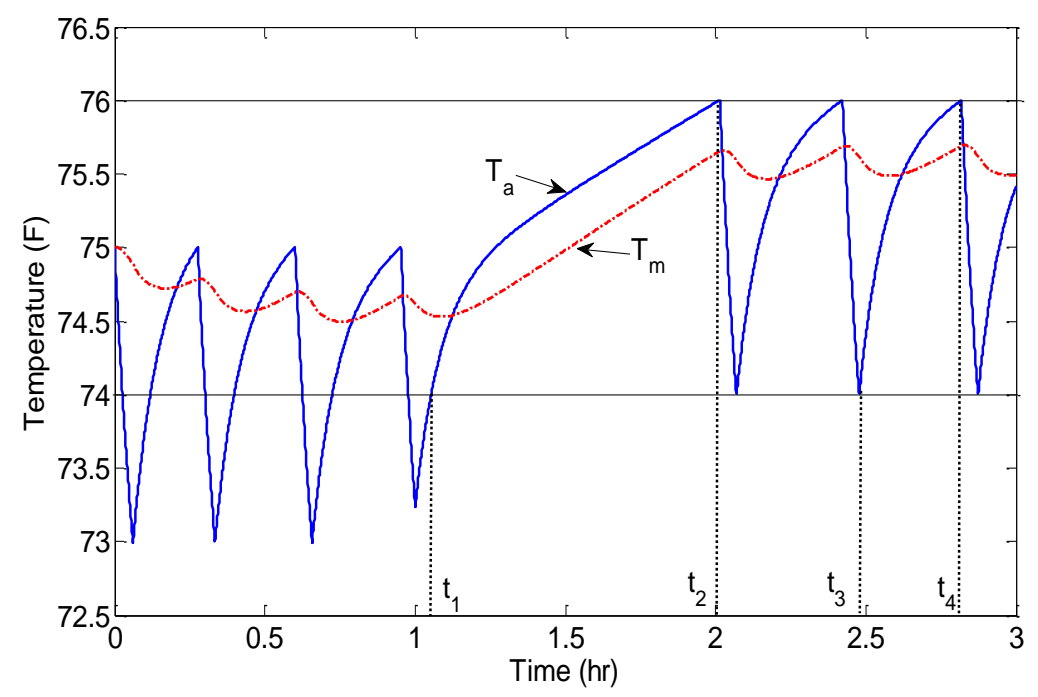

Figure 2 Coupled air and mass temperature dynamics 
Without considering $T_{m}$, the time derivative of $T_{a}$ would only depend on $T_{a}$ itself, which excludes the possibility of having different duty cycles as observed in Figure 2. In fact, the entire trajectory of $T_{a}$ as shown in Figure 2 cannot be accurately described by any first-order time-invariant ordinary differential equation (ODE) model. Therefore, a careful consideration of the coupled dynamics of $T_{m}$ and $T_{a}$, is necessary for obtaining a good aggregated model that can capture both the transient and steady state aggregated response for a population of TCLs.

\subsubsection{Effect of heterogeneity}

Another major factor that affects the aggregated transient response is the diversity in the parameters of the TCLs. It is well known that homogeneous load populations often exhibit strong oscillations, while a well-diversified load population will result in a natural damping and a more stable aggregate response [6], [7]. Although these observations are made mostly based on first-order TCL models, the second-order ETP model also yields similar behavior. Figure 3 compares the aggregated responses of 2000 homogeneous and heterogeneous TCLs under a setpoint change from $74^{\circ} \mathrm{F}$ to $75^{\circ} \mathrm{F}$ at time $t=2 \mathrm{hr}$. In the simulation, the ETP model parameters for the heterogeneous population are generated randomly according to the distribution described in [1], while the ETP parameters for the homogeneous population are chosen to be the average of the generated heterogeneous parameters.

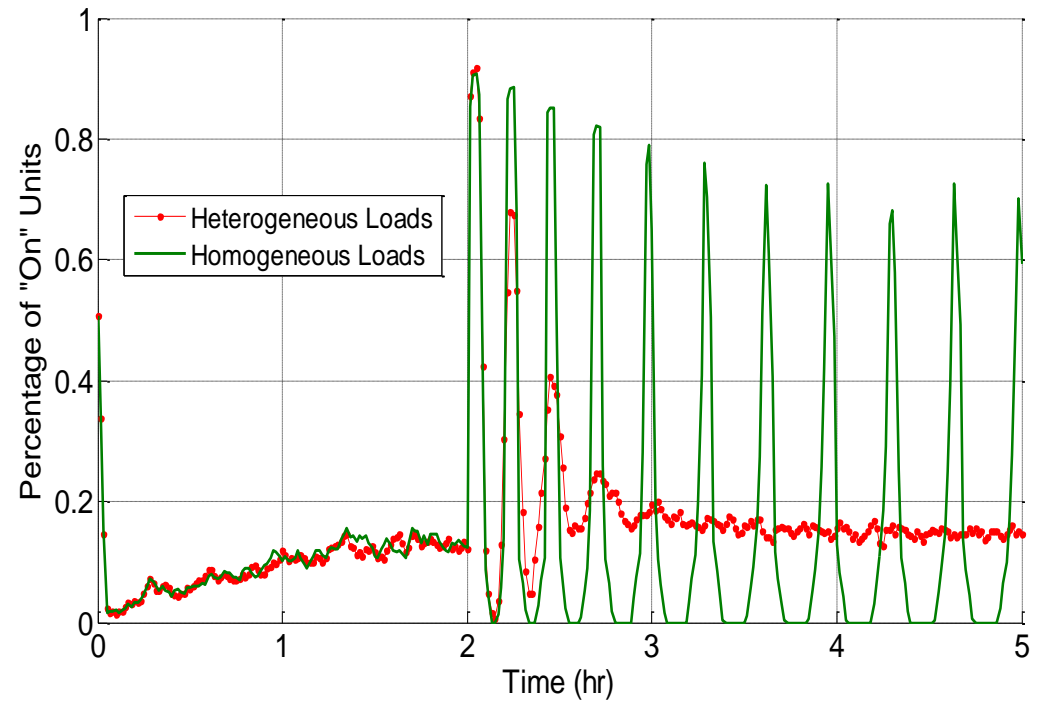

Figure 3 Aggregate response for homogeneous and heterogeneous TCLs

The evolution of the aggregated density of homogeneous TCLs can be described using partial differential equations (PDEs) as indicated in [6], [7]. In fact, many of the other proposed aggregate modeling approaches can also be viewed as particular numerical solutions to PDEs. The discretization in these numerical solvers often induces some artificial diffusion that smoothen sharp changes in the solution trajectory. This kind of diffusing effect acts similarly to the damping effect incurred by the load diversity. This is the main reason that many of the homogenous aggregate models developed deviate from the actual heterogeneous response less severely than what is observed in Figure 3. However, to accurately predict both transient and steady state aggregated responses, purely relying on the numerical errors in solving the PDE is not enough. A systematic way to deal with heterogeneity is necessary. 


\subsection{Design of reduced-order model for a population of HVAC units}

In this section, first a homogeneous aggregated model is derived in 2.2.1 based on the detailed second-order dynamics described in Sections 2.1 and 2.1.1. As mentioned in Section 2.1.2, heterogeneity among load population has a major effect on the aggregated response. The homogeneous aggregate model developed in the last subsection requires a fixed constant ETP model parameter vector $\theta$. In 2.2.2, a method is developed to account for heterogeneity where the parameter vector $\theta$ is a random variable with a known distribution. The development of the aggregated model is also presented in our papers [10], [11].

\subsubsection{Homogeneous aggregate model}

A detailed description of the second-order homogeneous aggregated model is presented next. Let $\left[T_{a, \min }, T_{a, \max }\right]$ and $\left[T_{m, \min }, T_{m, \max }\right]$ denote the air temperature range and mass temperature range, respectively. Divide these two intervals evenly into $n_{a}$ and $n_{m}$ bins. To account for the second-order effect caused by the mass temperature, consider a 2D discrete state space as shown in Figure 4, where $\operatorname{Bin}_{a}(i)$, $\operatorname{Bin}_{m}(j)$ represent the air and mass temperatures in bin $(i, j)$.

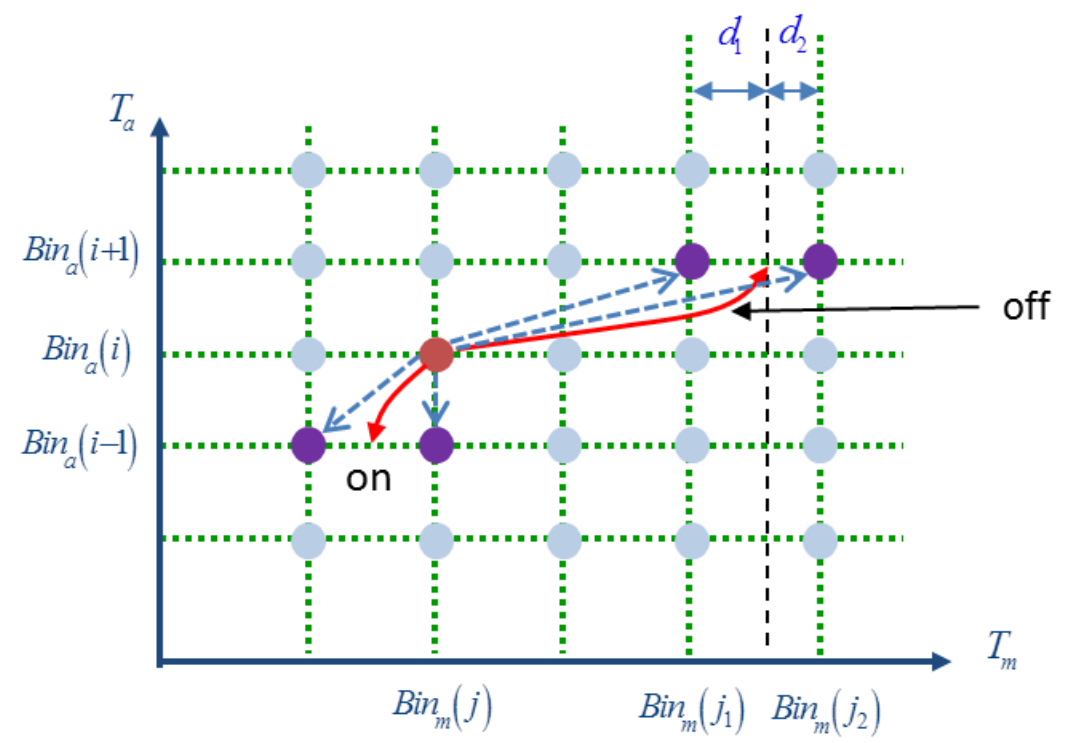

Figure 4 Illustration of 2D population flow, where the solid arrows represent the actual path the population moves, while the dashed arrows represent the corresponding approximated flow paths

Associated with each bin $(i, j)$, there is a (normalized) density function $p_{\text {on }}(i, j, t)$ representing the percentage (or probability) of the load population whose air temperatures are within the interval $\left[T_{a}(i)\right.$, $\left.T_{a}(i+1)\right]$, mass temperatures are in $\left[T_{m}(j), T_{m}(\mathrm{j}+1)\right]$, and power states are "On", at time $t$. The density for "Off" state $p_{\text {off }}(i, j, t)$ is defined similarly. One can think of $p_{\text {on }}(\cdot, t)$ and $p_{\text {off }}(\cdot, r)$ as $n_{a} \times n_{m}$ matrices. The two matrices can be vectorized as follows:

$$
P_{o n}(t)=\left[\begin{array}{c}
p_{o n}(1,1, t) \\
p_{o n}(1,2, t) \\
\vdots \\
p_{\text {on }}\left(n_{a}, n_{m}, t\right)
\end{array}\right] \text {, and } P_{\text {off }}(t)=\left[\begin{array}{c}
p_{\text {off }}(1,1, t) \\
p_{\text {off }}(1,2, t) \\
\vdots \\
p_{\text {off }}\left(n_{a}, n_{m}, t\right)
\end{array}\right]
$$


Define the total density state vector as

$$
q(t)=\left[\begin{array}{c}
P_{o n}(t) \\
P_{o f f}(t)
\end{array}\right]
$$

According to the above definition, each entry in $p_{\text {on }}(\cdot,, t)$ and $p_{\text {off }}(\cdot,, t)$ corresponds uniquely to an entry in the vector $q(t)$ as follows:

$$
\begin{aligned}
& (i, j, \text { "On" }) \leftrightarrow(i-1) \cdot n_{m}+j, \\
& (i, j, \text { "Off " }) \leftrightarrow n_{m} \cdot n_{a}+(i-1) \cdot n_{m}+j
\end{aligned}
$$

In the rest of this section, when no ambiguity arises, the $1 \mathrm{D}$ bin index is used interchangeably with the corresponding $2 \mathrm{D}$ bin index. The remainder of this section will be dedicated to deriving a linear dynamical system to describe the continuous time evolution for the state vector $q(t)$ of the form

$$
\frac{d q}{d t}=A q
$$

The matrix $A$ is typically a large sparse matrix. Denote the $(k, l)$ entry in matrix $A$, by $A_{k, l}$, which represent the rate (or speed) at which the population in bin $l$ is transported to bin $k$. The probability (or percentage) of the "On" units in the population denoted by $y(t)$ can be determined from the state vector $q(t)$ as follows:

$$
y(t)=\sum_{l}^{n_{a} n_{m}} p_{l}(t)
$$

To derive an expression of $A_{k, l}$, first transform the coupled first-order ODEs in the ETP model into a second-order ODE of $T_{a}$ as follows:

$$
a \ddot{T}_{a}+b \dot{T}_{a}+c T_{a}=d
$$

where

$$
a=\frac{C_{m} C_{a}}{H_{m}}, b=\frac{C_{m}\left(U_{a}+H_{m}\right)}{H_{m}}+C_{a}, c=U_{a}, d=Q_{m}+Q_{a}+U_{a} T_{o}
$$

With given initial conditions on air temperature $T_{a 0}$ and mass temperature $T_{m 0}$, the solution of this secondorder ODE is given by

$$
T_{a}\left(t ; T_{a 0}, T_{m 0}\right)=\alpha_{1} e^{r_{1} t}+\alpha_{2} e^{r_{2} t}+\frac{d}{c}
$$

where

$$
\begin{array}{lc}
r_{1}=\frac{-b+\sqrt{b^{2}-4 a c}}{2 a}, & r_{2}=\frac{-b-\sqrt{b^{2}-4 a c}}{2 a}, \\
\alpha_{1}=\frac{r_{2} T_{a 0}-\dot{T}_{a 0}-r_{2} d / c}{r_{2}-r_{1}}, & \alpha_{2}=T_{a 0}-\frac{d}{c}-\alpha_{1} \\
\dot{T}_{a 0}=\frac{H_{m}}{C_{a}} T_{m 0}-\frac{U_{a}+H_{m}}{C_{a}} T_{a 0}+\frac{Q_{a}+T_{o} U_{a}}{C_{a}} &
\end{array}
$$


Therefore, given the initial conditions $T_{a 0}$ and $T_{m 0}$, the solution trajectory for $T_{a}\left(t ; T_{a 0}, T_{m 0}\right)$ can be uniquely determined.

Consider a generic 2D bin $(i, j)$ that is not on the boundary of the control deadband as shown in Figure 4. If the population in this bin is "Off" and the system is operating in a cooling mode, then its air and mass temperature will increase and eventually the air temperature will reach the next level $\operatorname{Bin}_{a}(i+1)$. Let $t_{\text {off }}(i, j)$ be the time it takes to "transport" the population from bin $(i, j)$ to the next level $\operatorname{Bin}_{a}(i+1)$. This time can be found by solving the following nonlinear equation:

$$
T_{a}\left(t ; \operatorname{Bin}_{a}(i), \operatorname{Bin}_{m}(j)\right)=\operatorname{Bin}_{a}(i+1)
$$

where the function $T_{a}\left(\because \operatorname{Bin}_{a}(i), \operatorname{Bin}_{m}(j)\right)$ is the air temperature trajectory given in (3) with initial condition $T_{a 0}=\operatorname{Bin}_{a}(i)$ and $T_{m 0}=\operatorname{Bin}_{m}(\mathrm{j})$. Therefore, the population at bin $(i, j)$ is continuously being transported to the upper level at the rate $1 / t_{\text {off }}(i, j)$. Due to the discretization, when the air temperature reaches $\operatorname{Bin}_{a}(i)$, the mass temperature may not equal to any of the discrete bin values. As an approximation, the population flow is divided into two parts proportionally to $d_{2} /\left(d_{1}+d_{2}\right)$ and $d_{1} /\left(d_{1}+d_{2}\right)$ that reach the two neighboring bins $\left(i+1, j_{1}\right)$ and $\left(i+1, j_{2}\right)$, respectively, where $d_{1}$ and $d_{2}$ are the distances as illustrated in Figure 4. This leads to the following assignment of the corresponding entries in matrix $A$ :

$$
\begin{gathered}
A_{l_{1}, l_{1}}=-\frac{1}{t_{\text {off }}(i, j)} \\
A_{l_{3}, l_{1}}=\frac{1}{t_{\text {off }}(i, j)} \cdot \frac{d_{1}}{d_{1}+d_{2}} \\
A_{l_{2}, l_{1}}=\frac{1}{t_{\text {off }}(i, j)} \cdot \frac{d_{2}}{d_{1}+d_{2}}
\end{gathered}
$$

where $l_{1}, l_{2}, l_{3}$ are the $1 \mathrm{D}$ index corresponds to $\left(i, j\right.$, "Off'), $\left(i+1, j_{1}\right.$, "Off'), and $\left(i+1, j_{2}\right.$, "Off'), respectively. Note that a negative rate in (14) indicates the population is moving away from bin $l_{l}$ (only happens at the diagonal entries), while the positive rate in (15) means the population is flowing into bin $l_{2}$. The population density dynamics for the "On" state can be derived in a similar way.

It should be noted that special care needs to be taken for the bin that is on the boundary of the control deadband. For example, if bin $\left(i, j\right.$, “Off') is on the upper boundary of the deadband, i.e., $\operatorname{Bin}_{a}(i)=T \operatorname{set}+$ $L / 2$, then the inward flow rate can be computed in the same way as discussed above, while the outward flow rate is exactly equal to the inward flow rate and points directly into the corresponding bin in the "On" plane as shown in Figure 5. On the other hand, if bin ( $i, j$, "Off") is on the lower boundary of the deadband, then the outward flow rate is computed in the same way as the interior bins, while the inward flow rate equals the sum of the flow rate from one level below in the "Off" plane and the outward flow rate from the "On" plane. Once again, the case of flow rates for the bins with "On" power states can be handled similarly. The densities flow at different speeds (rates) at different locations which can be computed by solving the nonlinear expression given in (13). This proposed modeling framework allows the existence of densities that are outside the deadband during the transient response under a setpoint change. In addition, the flow rate matrix $A$ depends on the current setpoint of the population, and hence it will change according to the temperature setpoint. 


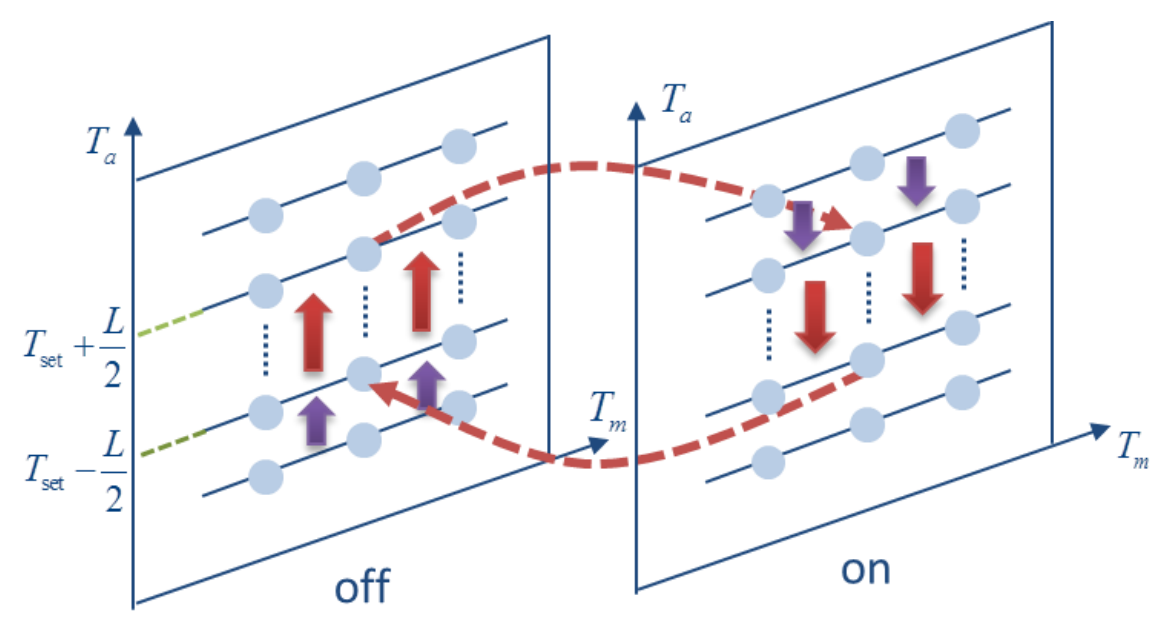

Figure 5 Illustration of 2D population flows and transitions between "On" and "Off" states

\subsubsection{Heterogeneous aggregate model}

The main idea behind the approach is to represent the randomly distributed ETP parameter vectors by a few representative vectors with appropriate weights. To this end, first generate a large number of parameter samples according to the known distributions. Denote these parameters by $\theta^{(1)}, \theta^{(2)}, \ldots, \theta^{(n)}$. A simple clustering technique like the $k$-means algorithm can be used to classify these parameters into $n_{c}$ clusters. Each cluster $i$ is associated with a center $\mathrm{CEN}(i)$ as well as the number of parameters that fall into this cluster, which is denoted by $N_{i}$. The probability (or relative weight) that the parameter vector of a randomly selected load falls into cluster $i$ is thus given by $w_{i}=N_{i} / n$. After obtaining the clusters, a homogeneous aggregated model is computed for each cluster $i$ by assuming all the loads have the same ETP parameter vector $\theta=\mathrm{CEN}(i)$. The resulting rate matrix is denoted by $A_{i}$. The entire load density $q(t)$ is then given by

$$
\begin{aligned}
& \dot{q}^{(i)}(t)=A_{i} q^{(i)}(t) \\
& q(t)=\sum_{i=1}^{n_{c}} w_{i} q^{(i)}(t) .
\end{aligned}
$$

The overall density trajectory $q(t)$ is the weighted average of the responses of all the clustered groups. When the number of clusters increases, the model is able to capture more heterogeneous effects at the price of increased model complexity. It will be shown in Section 2.4, that a significant performance gain can be achieved with only five clusters, making the proposed clustering strategy an effect way to cope with heterogeneity with acceptable computational overhead.

\subsection{Closed-loop controller design for HVAC unit aggregations}

Several model-based controllers of aggregated HVAC systems have been proposed in the literature [6], [7] and [17]. One common control approach is the so-called thermostat setback program. Under this approach, a central controller broadcasts universal setpoint changes to all the HVAC units. A small change in the setpoint of a large number of HVAC units can result in a significant change in the aggregated power. However, right after the change, a large undesirable rebound is often observed. The problem has been studied in [17], where some mathematical approximations of the transient dynamics are 
made. Under the assumption that the aggregate power can be measured and transmitted to the controller every few seconds, a closed-loop controller is developed that broadcasts the setpoint change signal to the population. Another widely used aggregate control approach is the so-called "toggling" control [7]. Under this approach, a central controller broadcasts a vector signal to all the devices to directly control their "On/Off" power modes. Each bin in the control signal has a probability of switching in a certain range of temperature. The temperature difference between the neighboring bins is about one or two orders smaller than the resolution of thermostats, which is typically around $1^{\circ} \mathrm{F}$. In this case, the information of the probability of switching contained in the control signal becomes unrecognizable at the device level. Another issue of this controller is that it also does not consider the "lockout" effect. When the compressor of a HVAC system is turned "Off", the air pressure in the chamber is high and a certain amount of time is needed for the pressure to even out. Restarting the compressor under pressure may cause physical damage to the device. Compressor time delay relays are typically installed for HVAC units that are controlled by electronic or programmable thermostats. These relays are used to keep the compressors in the "Off" state for a minimum off-time, for instance, five minutes. During the minimum-off time period (often called the compressor relaxation time), the HVAC unit is "locked" and any switching-on control signal will be ignored. The red dashed lines in Figure 6 illustrate the part of the state trajectory during which the unit is "locked" due to the spontaneous switching-off at the lower boundary of the control deadband. This property of HVAC systems will be referred to as the "lockout" effect in the rest of this paper. Any practical demand response study involving HVAC systems being controlled over short time periods ( seconds to minutes) should consider the lockout effect.

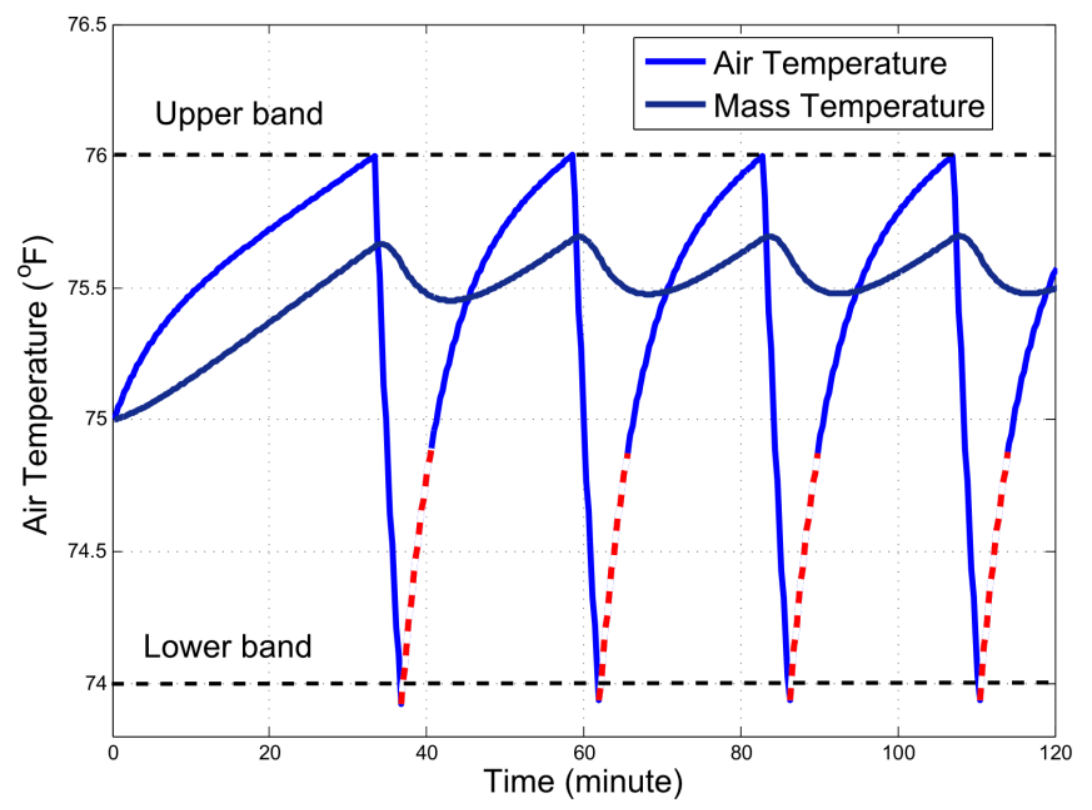

Figure 6 Air and mass temperature trajectories with setpoint $=75$, deadband $=1$

Both types of control strategies mentioned above control the aggregate load of HVAC units effectively under the assumption that every HVAC unit can respond to control signals in a short time period, for instance, several seconds. However, the units may not be able to respond due to the "lockout" effect. When a HVAC unit is "locked", it cannot be switched, even if the temperature is beyond the deadband when the setpoint is changed by the control signal. Similarly, the "locked" unit cannot be toggled by the broadcasted control signal. The effect restricts the possibility of controlling in that short 
period of time. If the "locked" populations are included in the aggregate model and kept from being switched, it would make the control of HVAC units in a short time period possible. This issue will be addressed next.

Under the proposed control strategy, the system operator will broadcast a scalar control signal $\alpha(k) \in[-1,1]$. Each HVAC unit will interpret the control signal probabilistically based on its own power mode. For example, if $\alpha(k)>0$ then each "Off" unit will have $\alpha(k)$ probability of turning "On" right away. On the other hand, if $\alpha(k)<0$ each "On" unit will turn off with probability $|\alpha(k)|$.

The evolution of the state vector $q(k)$ under the above control strategy can be described by the following modified aggregate model:

$$
q(k+1)=\left\{\begin{array}{l}
G B_{1}(\alpha(k)) q(k), \text { if } \alpha(k) \geq 0 \\
G B_{2}(\alpha(k)) q(k), \text { if } \alpha(k)<0
\end{array}\right.
$$

where $B_{1}(\cdot)$ and $B_{2}(\cdot)$ are matrix-valued functions of the control $\alpha(k)$ defined as follows:

$$
B_{1}(\alpha)=\left[\begin{array}{cc}
I_{n} & \alpha I_{n} \\
0 & (1-\alpha) I_{n}
\end{array}\right], \quad B_{2}(\alpha)=\left[\begin{array}{cc}
(1+\alpha) I_{n} & 0 \\
-\alpha I_{n} & I_{n}
\end{array}\right],
$$

for an arbitrary control value $\alpha(k) \in[-1,1]$ Here, $I_{n}$ denotes the identify matrix of dimension $n$. The density dynamics under the proposed control strategy $\alpha(k)$ without considering the lockout effect are described in (18). As described before, the "locked" populations cannot be turned "On" until the end of the minimum off-time. To account for this restriction, we introduce another state vector $q^{L}=\left[q_{1}^{L}, \ldots, q_{n L}^{L}\right]^{T}$ whose dimension $n_{L}=\tau / \Delta t$, where $\Delta t$ is the discrete time unit. The $i^{\text {th }}$ entry of $q^{L}$ is the amount of locked population that will be released after $n_{L}-i+1$ discrete time units. The total locked population is given by $y^{L}=C^{L} q^{L}$ where $C^{L}$ is a row vector of dimension $n_{L}$ with all entries equal to 1 . Notice that the lockout population is a subset of the "Off" population and cannot be switched "On". At any time $k$, the actual population that can be freely switched "On" is given by:

$$
\hat{q}(k)=\left[\begin{array}{cc}
I_{n} & 0 \\
0 & I_{n}\left(1-\frac{y^{L}(k)}{Q_{\text {off }}(k)}\right)
\end{array}\right] q(k)
$$

where $Q_{\text {off }}$ is the summation of all the populations in “Off" state. It is beneficial to think of the entire evolution during each discrete time period as two sequential stages.. During the first stage, the population switches according to the control signal $\alpha(k)$, while during the second stage, the population evolves naturally according to the state-transition matrix $G$. Denote $q^{+}(k)$ as the density vector after the first stage of time step $k$. Then, with consideration of the lockout effect, the modified aggregate model is given by:

if $\alpha(k) \geq 0$

$$
\left\{\begin{array}{l}
q^{+}(k)=B_{1}(\alpha(k)) \hat{q}(k)+(q(k)-\hat{q}(k)) \\
q(k+1)=G q^{+}(k) \\
q^{L}(k+1)=G^{L} q^{L}(k)+B^{L} \cdot S \cdot q^{+}(k),
\end{array}\right.
$$


if $\alpha(k)<0$

$$
\left\{\begin{array}{l}
q^{+}(k)=B_{2}(\alpha(k)) q(k) \\
q(k+1)=G q^{+}(k) \\
q^{L}(k+1)=G^{L} q^{L}(k)+B^{L} \cdot\left(|\alpha| \cdot y(k)+S \cdot q^{+}(k)\right)
\end{array}\right.
$$

where $B^{L}=[1,0, \ldots, 0]^{T}$ and $S$ is a $1 \times 2 n$ matrix, such that is the total amount of loads that will turn "off" and become locked if the system evolves autonomously (without additional control) from $q^{+}$during the second stage of time step $k$. The matrix $S$ can be derived as follow:

$$
S=[\underbrace{0, \ldots, 0}_{n}, 1, \ldots, 1] G\left[\begin{array}{ll}
I & 0 \\
0 & 0
\end{array}\right]
$$

The $G^{L}$ matrix in (20) and (21) is an $n_{L} \times n_{L}$ matrix that determines the evolution of the lockout population. It is given by:

$$
G^{L}=\left[\begin{array}{ccccc}
0 & 0 & \cdots & \cdots & 0 \\
1 & 0 & \ddots & \ddots & \vdots \\
0 & 1 & \ddots & \ddots & \vdots \\
\vdots & \ddots & \ddots & \ddots & \vdots \\
0 & \cdots & 0 & 1 & 0
\end{array}\right]
$$

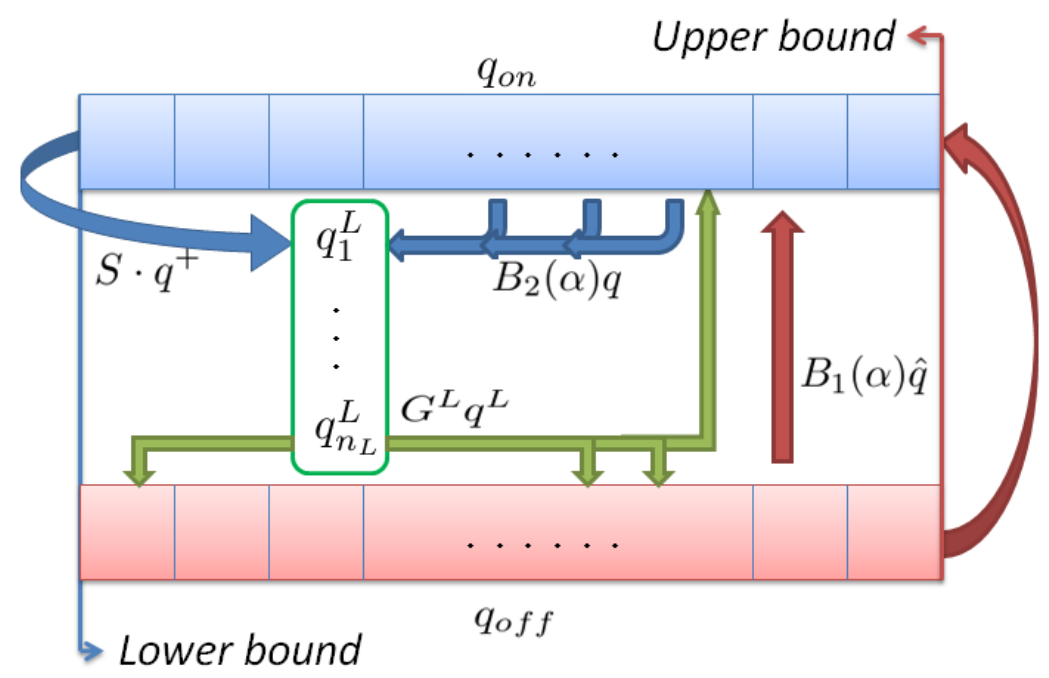

Figure 7 Illustration of density evolution with consideration of the lockout effect

Figure 7 illustrates the state transition of the modified aggregate model. In the proposed model, unlike other aggregate models without considering lockout effect, the populations in the "On" state never flow directly into "Off" state. Instead, the population first flows into $q_{1}^{L}$, when hitting the lower boundary of the deadband or being toggled by the control signal. After evolving to $q_{n L}^{L}$, the population has been "locked" for at least the minimum off-time $\tau$, and is thus released to the unlocked "Off" state. One feature is that even if every $q_{i}^{L}$ is a scalar, the populations in $q_{i}^{L}$ still evolves according to the state-transition matrix $G$. As a result, when the populations are released from the "locked" states by $G^{L} q^{L}$, each of them 
flows back to the bin corresponding to its temperature and "On/Off" state. The cycle completes once the population returns back to $q_{o n}$ when reaching the upper boundary of the deadband or being toggled "On" by the control signal $\alpha$.

The control signal $\alpha$ is designed to match the real density of "On" state $y_{\text {real }}$ for a given desired trajectory $y_{\text {ref }}$. Since the $y_{\text {model }}(k+1)=C q(k+1)$ is considered to be a good estimation of $y_{\text {real }}(k+1)$, we use the MATLAB function "fzero" in equation and to find the $\alpha(k)$ such that $y_{\text {ref }}(k+1)-y_{\text {model }}(k+1) \cong 0$. $y_{\text {real }}(k+1)$ is then expected to follow the $y_{\text {ref }}(k+1)$ as $y_{\text {model }}(k+1)$ does. The total amount of "locked" populations, considered in the modified aggregate model is kept from switching by the control signal $\alpha$. This design allows each HVAC unit to ignore the commands of turning "On" before the end of the minimum off-time. Therefore, the central controller is able to send control signals to the device level in a short period without concerns of forcing some populations to start overloading. This prevents an inaccurate estimation of the aggregate load due to the "locked" populations, which are not turned "On". One issue is that if too many devices are "locked", the number of available controllable units may be too few. The problem would be resolved when a large enough population size of HVAC is available.

\subsection{Simulation results}

This section presents simulation results validating the performance of the aggregated model and the control strategies discussed in Sections 2.2 and 2.3. A more detailed validation of the aggregated model and closed-loop control strategies is given in Appendix A.1.

\subsubsection{Performance of aggregated model}

The aggregate model is validated against realistic simulations produced by an open-source tool GridLAB-D. GridLAB-D is capable of simultaneously simulating thousands of unique buildings using the ETP model to create a diverse population of building and HVAC system parameters [13]-[21] . However, the simulations can often take a substantial amount of time and computing resources, especially as the population size increases. In the following experiments, 2000 building ETP models were created and simulated for a single day. The ETP model parameters, such as $U_{a}, C_{m}, C_{a}, H_{m}$, and $Q_{h}, Q_{i}, Q_{s}$, are determined by the various physical parameters of the building such as floor area, glazing layers and material, infiltration volumetric air exchange rate, area per floor, to name a few. The readers are referred to [10] for a detailed description of these physical parameters and their relations to the ETP model parameters. In the simulations, each of the 2000 houses is assigned to a set of physical parameters generated randomly around their nominal values (see [10]) with a certain amount of variance to provide diversification. The physical parameters then in turn determine the ETP model parameters used in both the GridLAB-D and the proposed aggregate model. The internal setpoints of all the houses were modified simultaneously at various times to test the performance of the aggregate model for demand response actions. For each of the 2000 houses, the initial air and mass temperatures and the "On/Off" state are generated randomly over the initial control deadband. GridLAB-D is then used to simulate each of the houses starting from their initial conditions, which are computed based on the corresponding ETP model parameters. To simulate the proposed aggregate model, the 2000 ETP models are classified into $n_{c}$ clusters as described in Section 2.2.2. For each cluster $i$, its center $\operatorname{CEN}(i)$ is used to compute the rate matrix $A_{i}$. The initial population density for each cluster is the same, which can be obtained by computing the percentage of the initial conditions of the 2000 houses that fall into each of the $1 \mathrm{D}$ bins according to 
the bin indexing convention described in (6). After obtaining the initial density vector $q^{(i)}$, the overall aggregate response can be simulated according to (17). Once the overall density is obtained, the percentage of the "On" units in the population can be computed according to (8).

The proposed heterogeneous aggregate model is tested through a simple form of demand response, a thermostat setback program, under various scenarios. In these simulations, the aggregate model uses 50 bins (i.e., $n_{a}=n_{m}=50$ ) and $n_{c}=5$ clusters. Figure 8 shows a comparison of the percentage of "On" units between the aggregate model and the GridLAB-D simulations. In these two cases, all the HVAC units are participating in a thermostat setback program where the setpoints are simultaneously shifted from $75^{\circ} \mathrm{F}$ to $76^{\circ} \mathrm{F}$ at time $t=3 \mathrm{hr}$. It can be seen that the aggregate model is able to capture both the transient and the steady state responses.

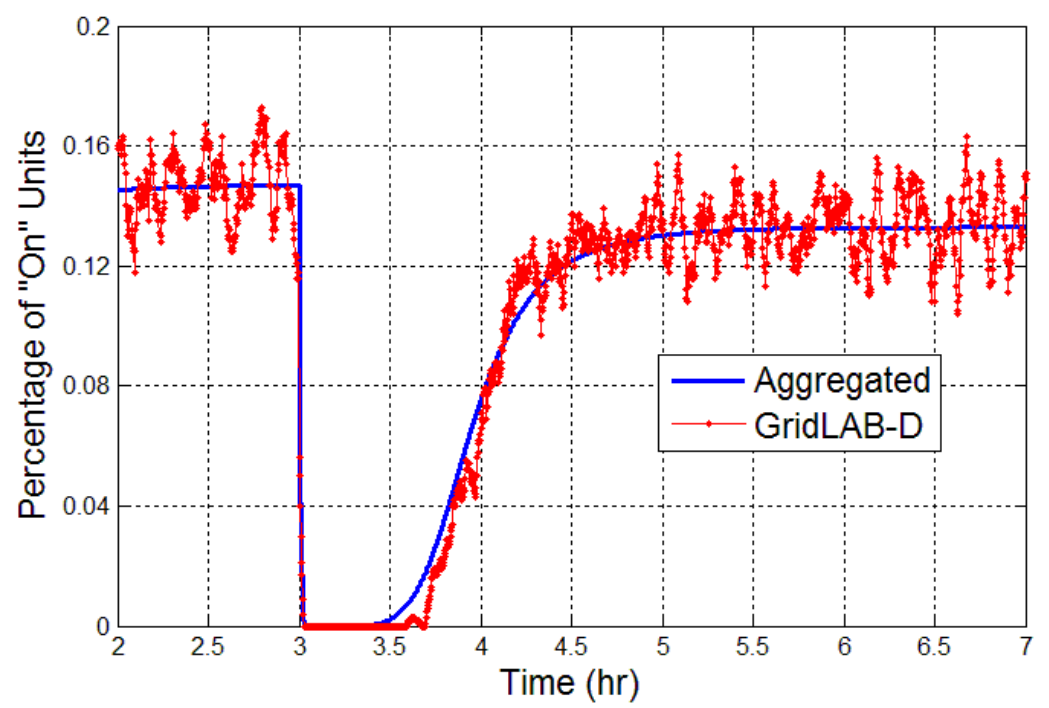

Figure 8 Aggregated response with setpoint change from 75 to 76 degrees

It is well known that thermostat setback programs may lead to the "rebound" effect or an immediate spike in power demand when the setpoint is changed back to the normal setting. This is illustrated in Figure 9 and Figure 10, where the setback program is released at time $t=3 \mathrm{hr}$. and the setpoints are changed from $75^{\circ} \mathrm{F}$ back to $74^{\circ} \mathrm{F}$ and $70^{\circ} \mathrm{F}$, respectively. In both cases, the aggregate model is able to accurately capture the strong transient response due to the loss of diversification after the setpoint change. With a large population size, such a strong transient rebound may affect the stability of the overall power system. Therefore, the ability to capture accurately the rebound effects is crucial for incorporating demand response in the grid. 


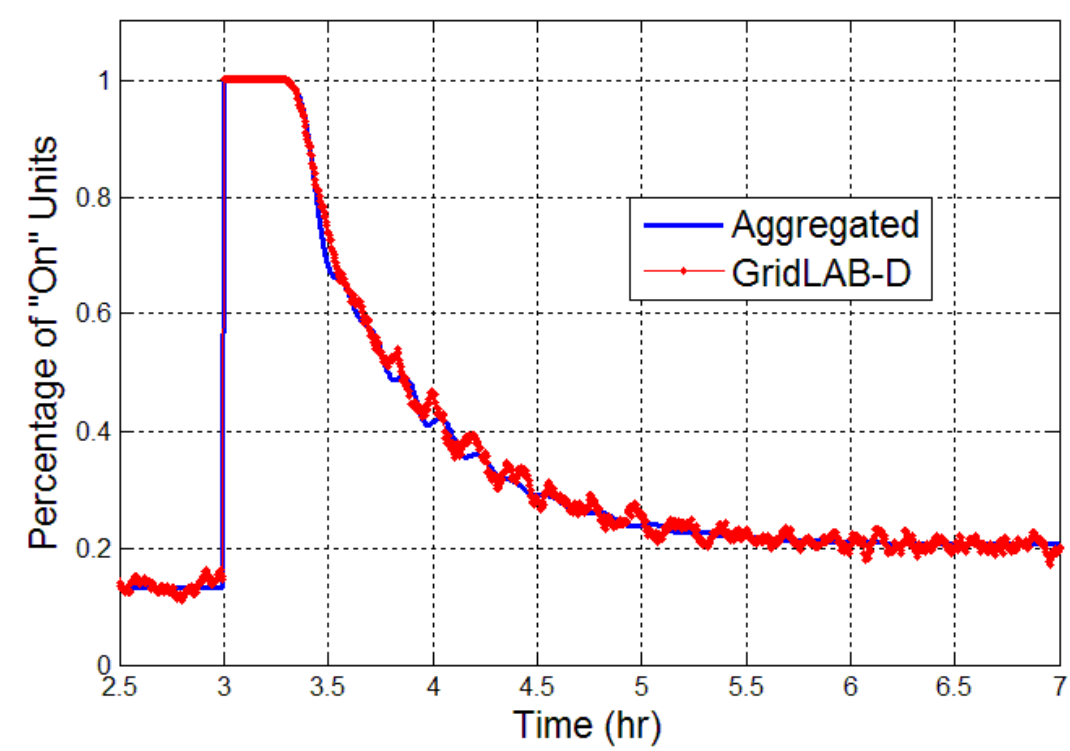

Figure 9 Aggregated response with setpoint change from 75 to 70 degrees

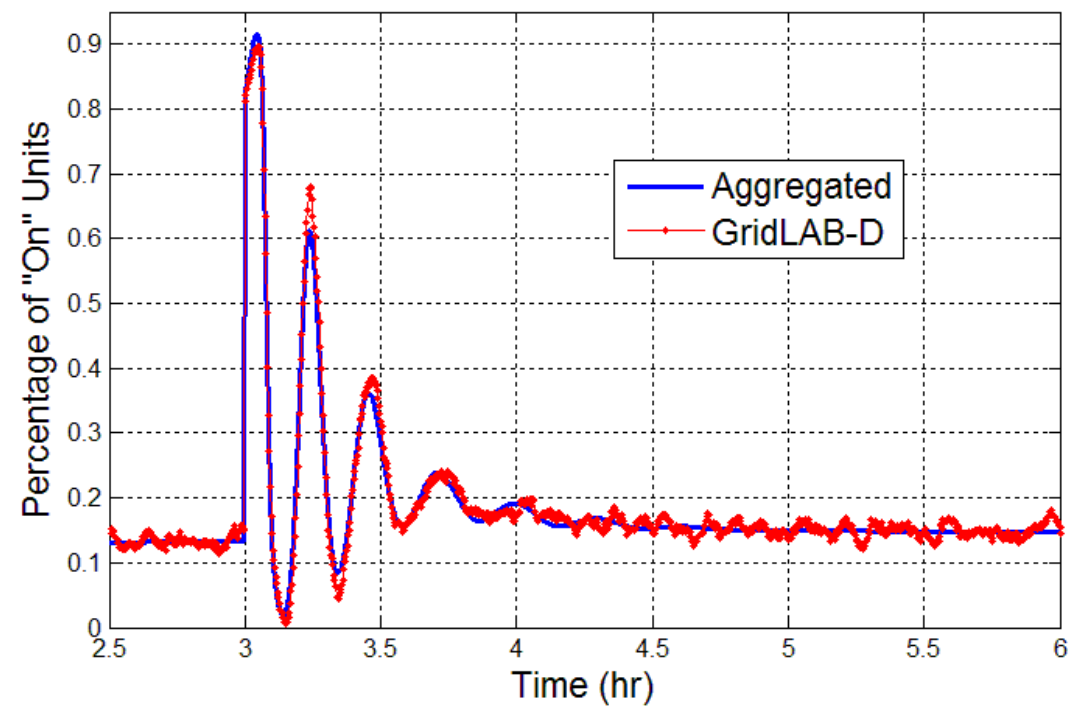

Figure 10 Aggregated responses with setpoint change from 75 to 74 degrees

The aggregate model can also be used to study the effect of sequential setpoint scheduling. Figure 11(a) compares the aggregate model and GridLAB-D simulations when the setpoint is changed from $75^{\circ} \mathrm{F}$ to $74^{\circ} \mathrm{F}$ and then back to $75^{\circ} \mathrm{F}$. The changes of the setpoints are applied after the population reaches its steady state. It is observed that the aggregate model is able to accurately reproduce the collective behavior over the 10-hour simulation horizon, which represents an improvement over existing methods that have been developed that can only produce short-term open-loop prediction. In addition, the proposed aggregate model is also tested under a sequence of setpoint changes that are applied before the population reaches its steady state as shown in Figure 11(b). It is impressive to observe that the aggregate model is able to capture all the dynamics even under these premature setpoint changes. It is important to notice that the response of the last setpoint change from $75^{\circ} \mathrm{F}$ to $70^{\circ} \mathrm{F}$ is quite different from the one in Figure 9. Such a difference is due to the fact that the setpoint change in Figure 11 happens before the 
population reaches the steady state distribution, which highlights the importance of the underlying density distribution on the overall response of the system. A good performance in this case shows the potential of the proposed model in dealing with rather complex demand response scenarios.

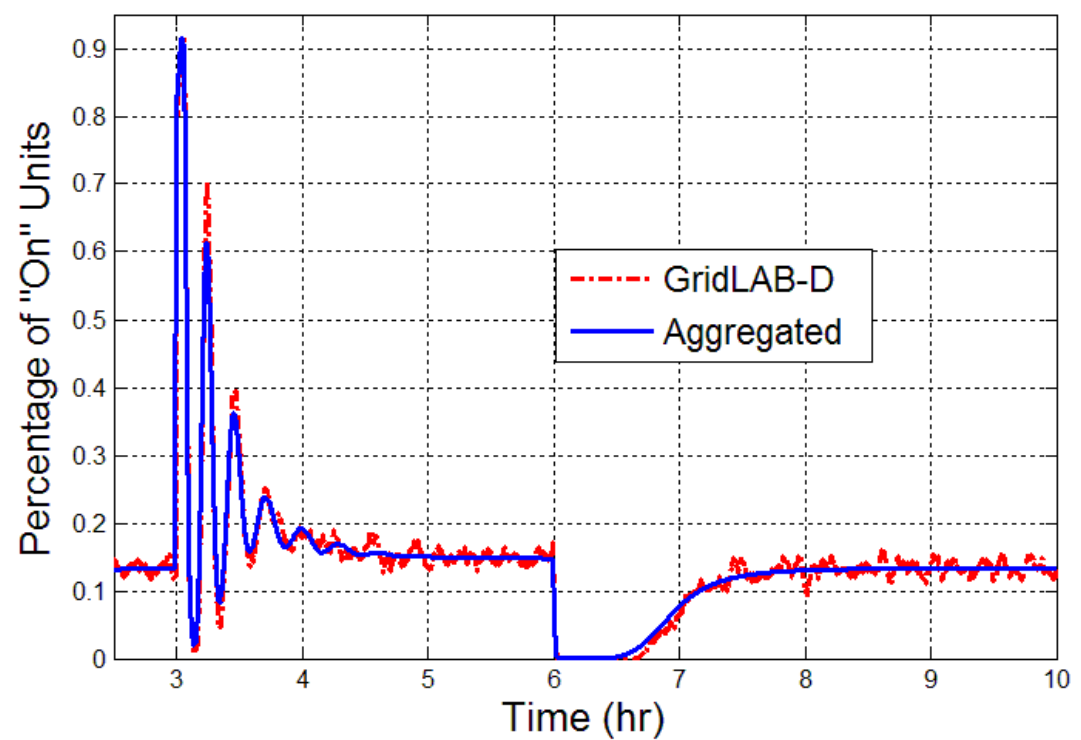

(a)

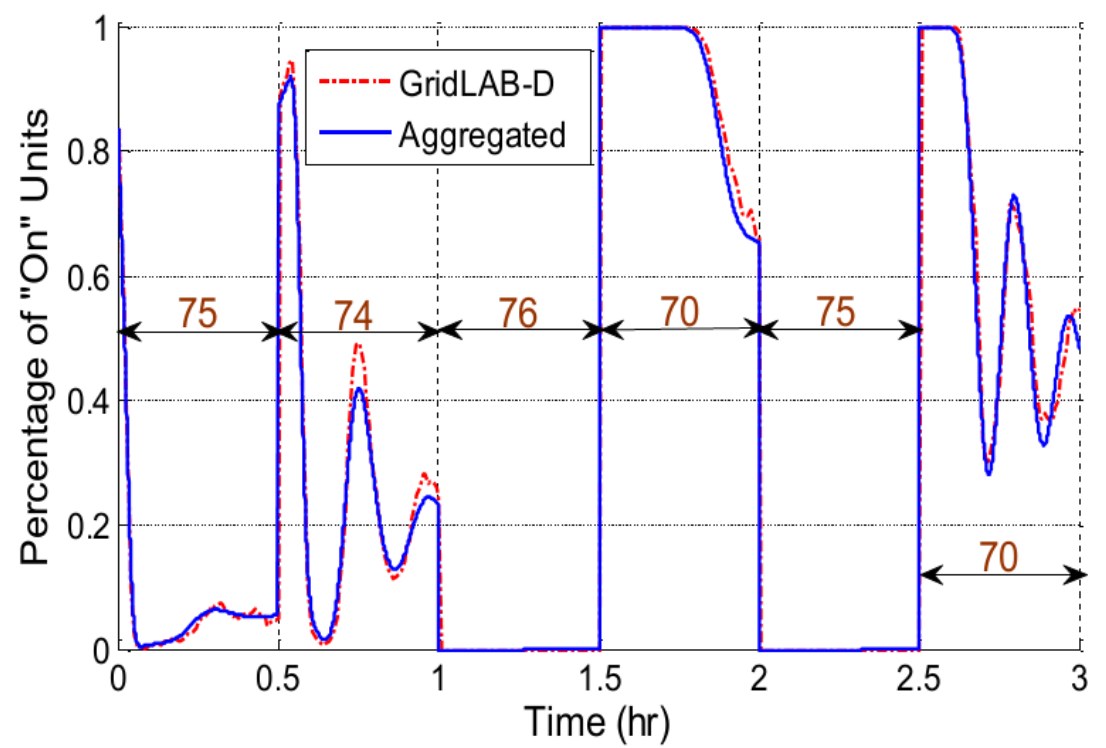

(b)

Figure 11 Aggregate response under sequential setpoint changes. (a) Setpoint change from 74 to 75 after the population reaches the steady state, (b) Sequence of setpoints applied before the population reaches the steady state

Another unique feature of the proposed aggregate modeling framework is the ability to achieve a desired performance and complexity tradeoff by adjusting the number of bins and clusters in the model. This feature is illustrated through several simulations as shown in Figure 12 and Figure 13. In these simulations, the air temperature setpoint is changed from $75^{\circ} \mathrm{F}$ to $74^{\circ} \mathrm{F}$, which produces large oscillations 
during the transient period. Figure 13 shows the corresponding aggregated responses generated by the proposed models with 8 clusters and different number of discretization bins. It is observed that with only 3 bins, the model is able to match the steady state response accurately and capture the trend of the transient response. As the number of bins increase, the transient response is matched more accurately. In particular, the model is able to predict accurately all of the main oscillations with 60 bins. The ability to always match the steady state response and to be able to tradeoff transient performance with complexity, distinguishes the proposed model from many of the existing aggregate models in the literature.

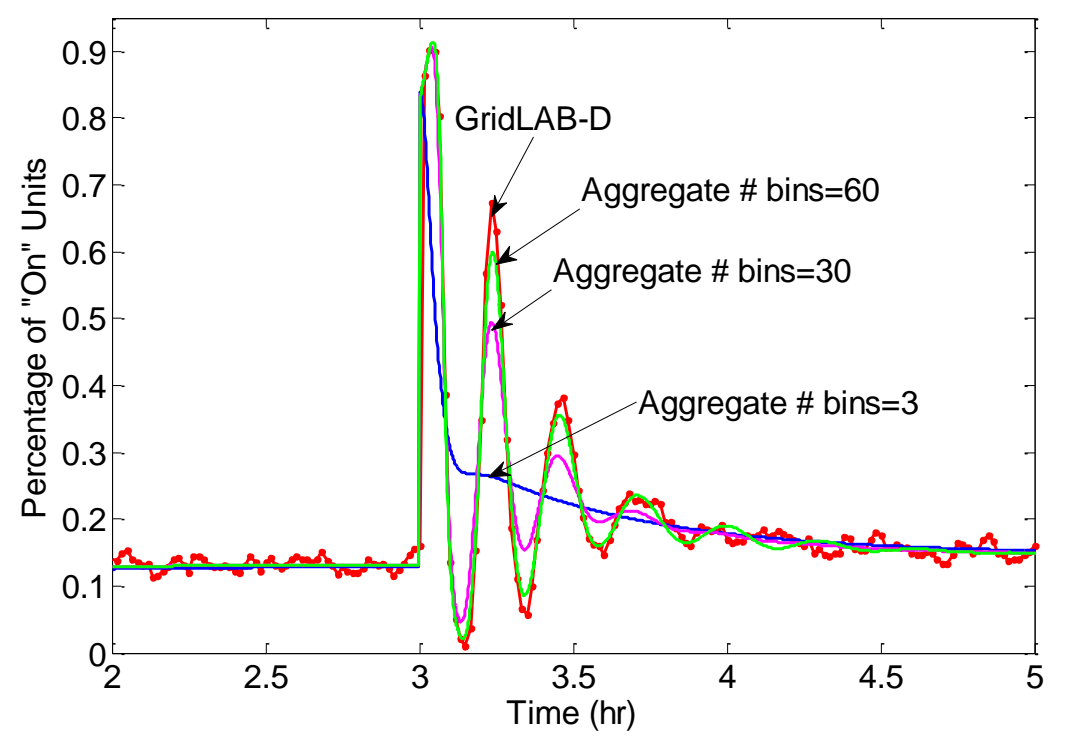

Figure 12 Aggregate responses with different number of discretization bins under a setpoint change from $75^{\circ} \mathrm{F}$ to $74^{\circ} \mathrm{F}$ (with 8 clusters)

Another parameter that affects both the performance and the model complexity is the number of clusters $n_{c}$. Figure 13 shows the model performance under different number of clusters. When one cluster is used, the proposed model degenerates to a homogeneous aggregate model which deviates significantly from the response generated by the detailed GridLAB-D simulation. This issue is successfully addressed by the proposed clustering technique. As shown in Figure 13, the model performance improves significantly as the number of clusters increase from 1 to 5 . The performance can be further improved when $n_{c}$ is equal to 10 , but the improvement is not as significant as before.

Based on the above results, the proposed aggregate model provides a general framework for analyzing different demand response applications. For a setpoint controller design, one may want to use less number of bins and clusters to simplify the design process while still capturing the steady state and the main transient behavior. For stability analysis of the distribution system under demand response, one may need to use more bins to capture all the transients. Furthermore, the time taken to simulate a 7-hour response with 2000 houses takes less than one minute for the aggregate model with 60 bins and 5 clusters, but more than 20 minutes for GridLAB-D with 2000 houses. If the number of bins is reduced to 10 or less, the simulation of the aggregate model only takes a fraction of second. The savings in the computational time will become more significant as the population sizes increase. 


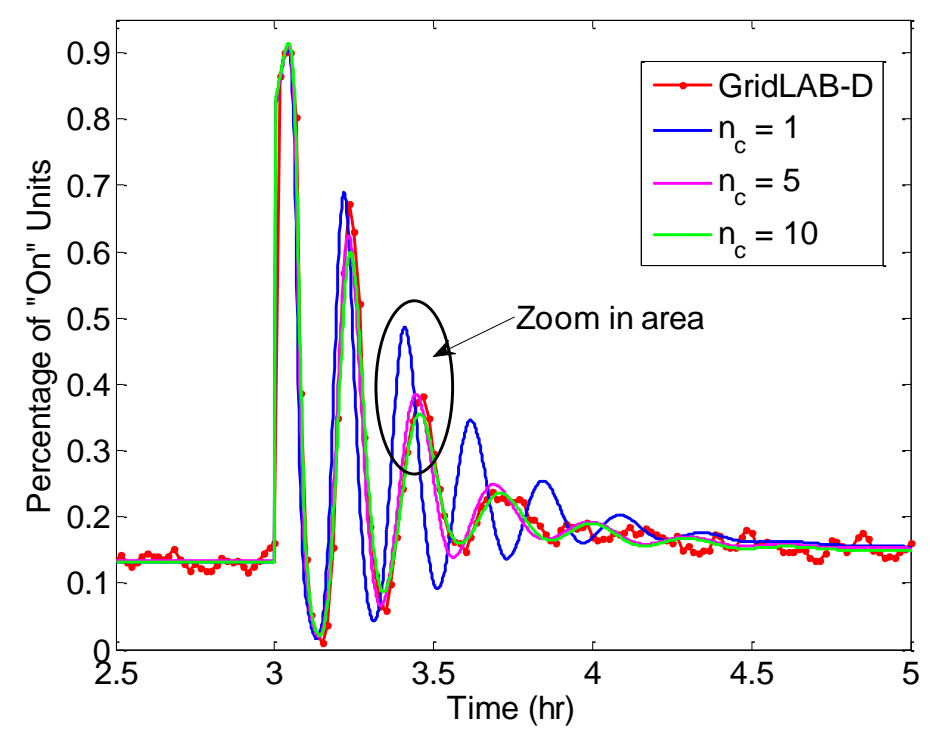

(a)

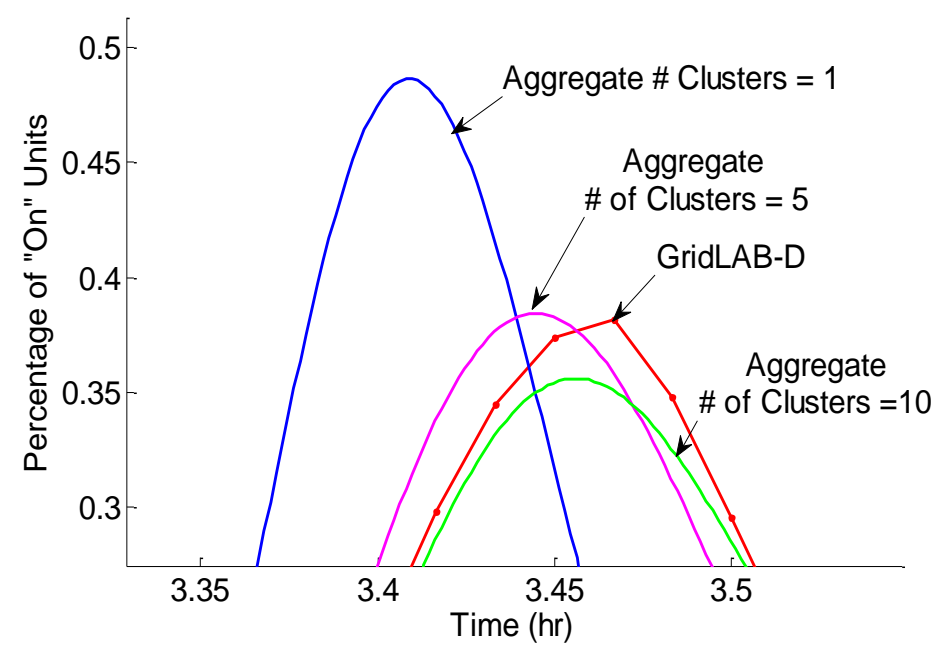

(b)

Figure 13 Aggregated responses with different number of clusters under a setpoint change from $75^{\circ} \mathrm{F}$ to $74^{\circ} \mathrm{F}(\#$ bin $=60)$

The lockout effect plays an important role when HVAC units are subjected to the control signal $\alpha$. The simulation compares the performance of predicting the real aggregate output between the modified aggregate model and the original one with no consideration of "locked" populations. The real aggregate output is obtained by simulating each HVAC unit with its ETP model. As shown in Figure 14, the modified model matches the real aggregate load better than the original one, especially when $\alpha$ is positive. The original model overestimates the "On" populations when $\alpha$ is positive because some "locked" populations are assumed to be turned "On", while this is actually not the case. On the contrary, the modified aggregate model estimates the "locked" populations and aggregated power accurately. 


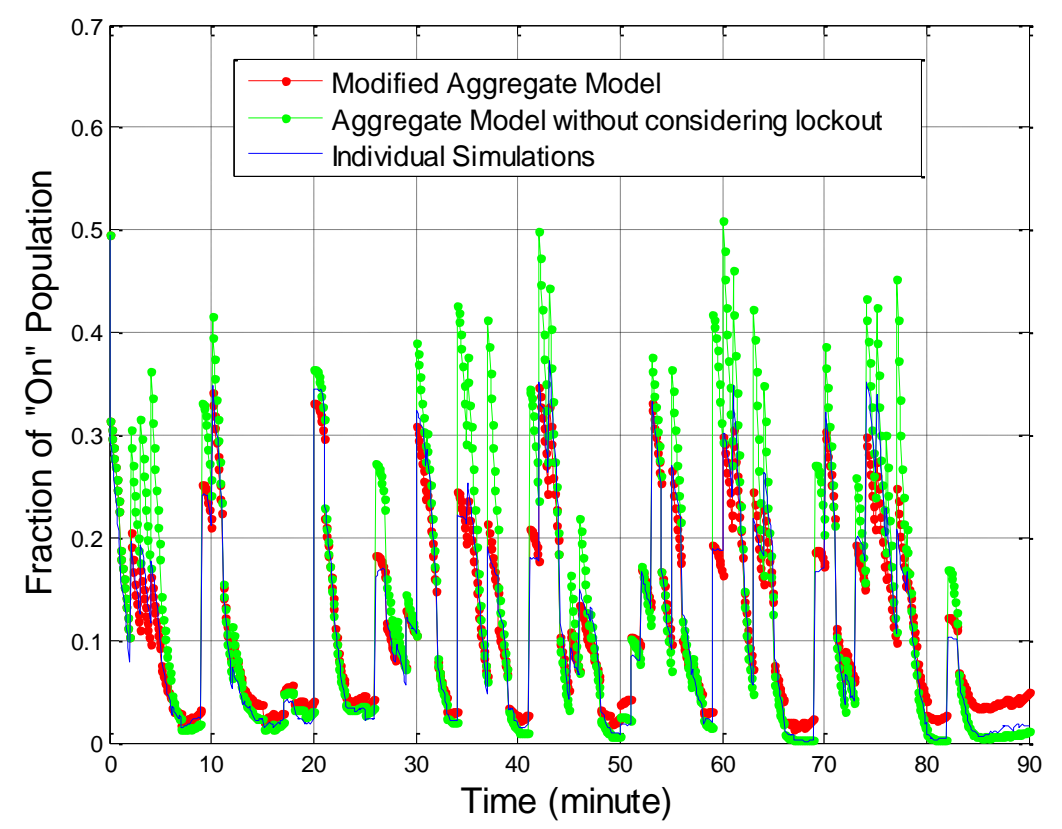

(a)

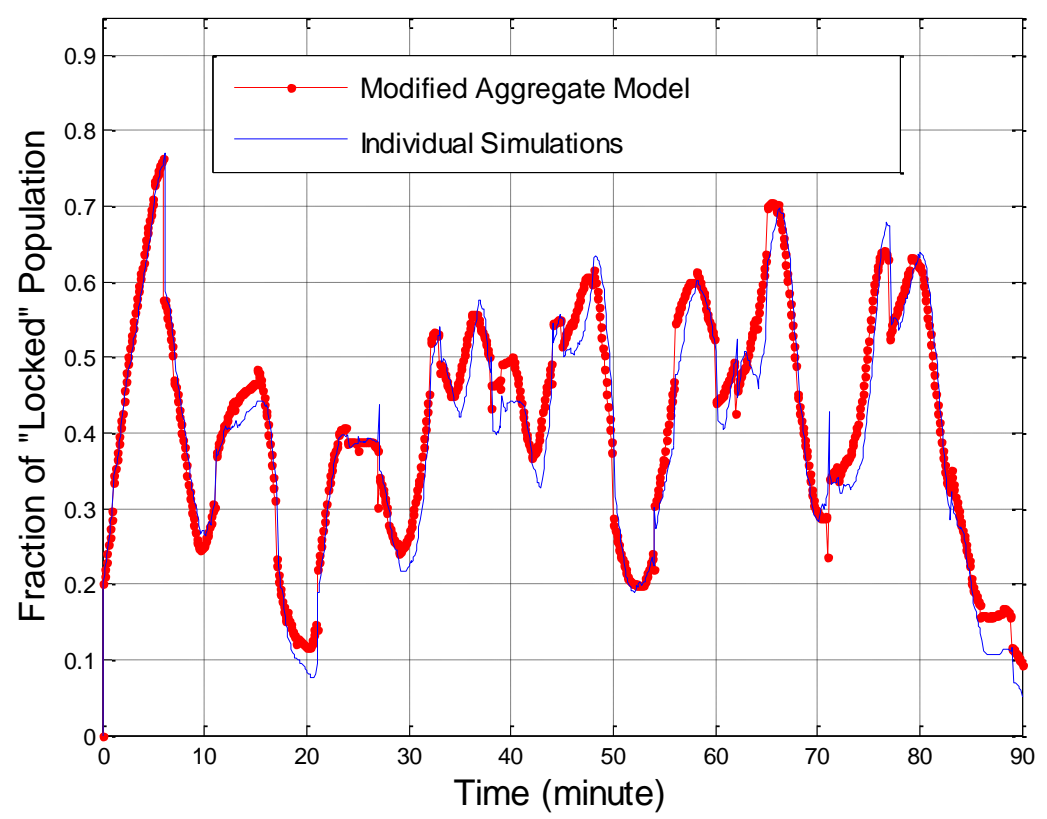

(b)

Figure 14 Aggregated model response including lock-out effect

\subsubsection{Performance of aggregated control strategies}

In this section, simulation studies were performed to illustrate the benefits of the proposed aggregated control strategy. Five thousand sets of physical building parameters are generated, which are randomly distributed around their nominal values with a certain amount of variance. Based on the relationship between the physical parameters and the ETP model described in [10], 5000 sets of ETP model 
parameters are obtained and used in all the simulations in this section. The proposed aggregated control strategy is then tested against different scenarios of possible demand response services, including regulation and load reduction. In those case studies, HVAC units are assumed to consume $5 \mathrm{~kW}$ on average.

\subsubsection{Direct load control}

Direct load control (DLC) is a demand-side management program that curtails the power demand of consumers during peak demand period. The same 5000 HVAC units are assumed to participate in a DLC program. Consider the scenario where after $1 \mathrm{hr}$., $50 \%$ of the units are switched "Off" directly and kept in the "Off" state until 30 minutes later. By the end of the load reduction period, they are released simultaneously at $1.5 \mathrm{hr}$. The effect of cold load pickup is then observed, which in extreme cases may damage distribution equipment. We address this issue by controlling the load based on the modified aggregate model for not only the load reduction period, but also 30 minutes afterward as shown in Figure 15. During the load reduction, aggregate load of HVAC units is controlled at $1.7 \mathrm{MW}$, instead of the steady state 3.5 MW. For the following 30 minutes after the end of the load reduction, the load is controlled at 4.2 MW. The value is slightly higher than the steady state for 30 minutes to provide a room to spread out the turning "On" time of those "Off" units from the load reduction. As a result, the "Off" populations are kept from turning "On" simultaneously and a smooth aggregate response is obtained. Another benefit of the second control strategy is that the temperature of every user is kept between the dead-band. The first DR approach made those devices turned "Off" for at least 30 minutes, which will cause too much discomfort to the users.

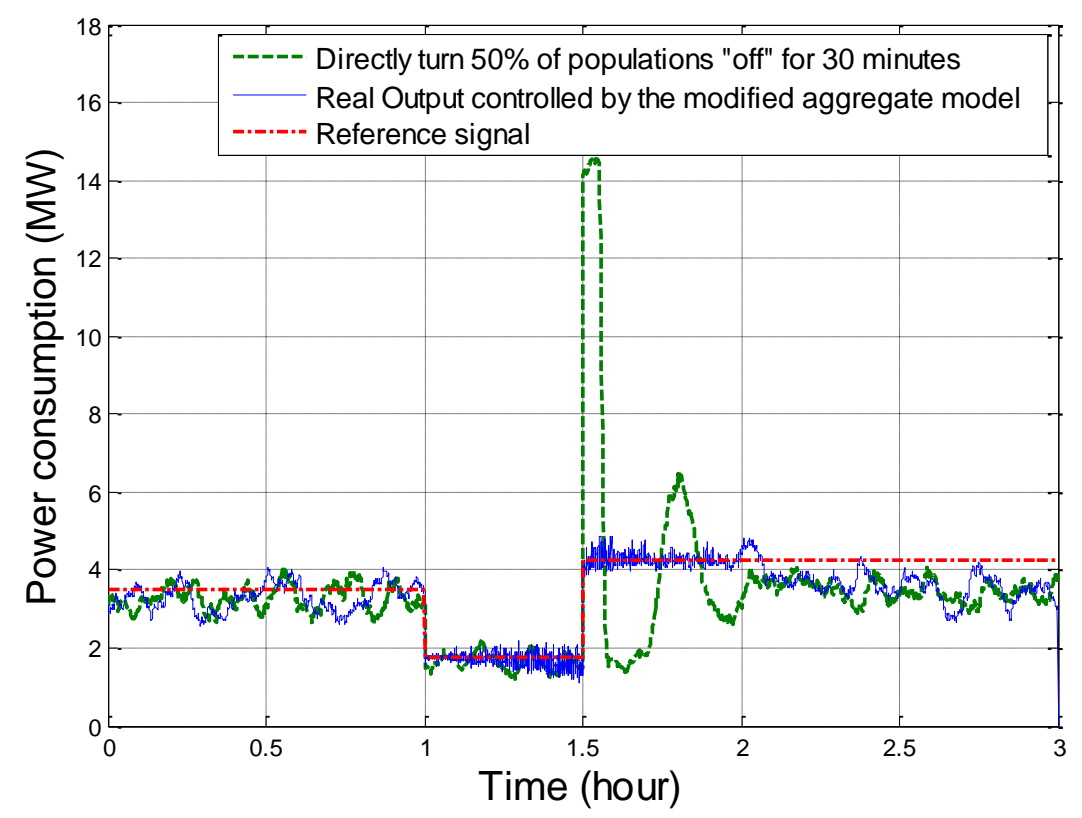

Figure 15 Direct load control for 30 minutes 


\subsubsection{Regulation services}

Since HVAC units can be turned on and off very quickly. If they are not subject to lockout, they have the potential to provide fast response as desired by regulation services. In this subsection, we assume 5000 HVAC units are incorporated to provide the regulation service in Pennsylvania-New JerseyMaryland Interconnection (PJM), which is one of the main regional transmission organizations (RTOs) in North America. Regulation is a service which manages a continuous balance of electricity generation and consumption in response to the changes in electricity use. In PJM, the total required regulation is roughly one percent of the regional load. The readers are referred to [18] for the details of regulation service. The 5000 HVAC units are collected to provide up-regulation and down-regulation with capacity of $2.5 \mathrm{MW}$. When the setpoint of those populations is $75^{\circ} \mathrm{F}$, the average aggregate power consumption is around 3.5 MW. Therefore, 3.5 MW is taken as a baseline of the $5000 \mathrm{HVAC}$ units in the following simulation. The details of the methods on estimating the baseline load are in [19]. With the baseline given, the HVAC units are then assigned to follow a dynamic regulation signal, which is a test signal downloaded from the PJM website [20]. The signal was adjusted such that the regulation range is $2.5 \mathrm{MW}$. During the regulation period, each HVAC unit receives control signals $\alpha$ and sends the measured power consumption data to the central controller every 15 seconds. Figure 16 shows that the controlled aggregated loads can follow the reference signal very accurately. In the simulation, each load is assumed to have a 5-minute lock-out time.

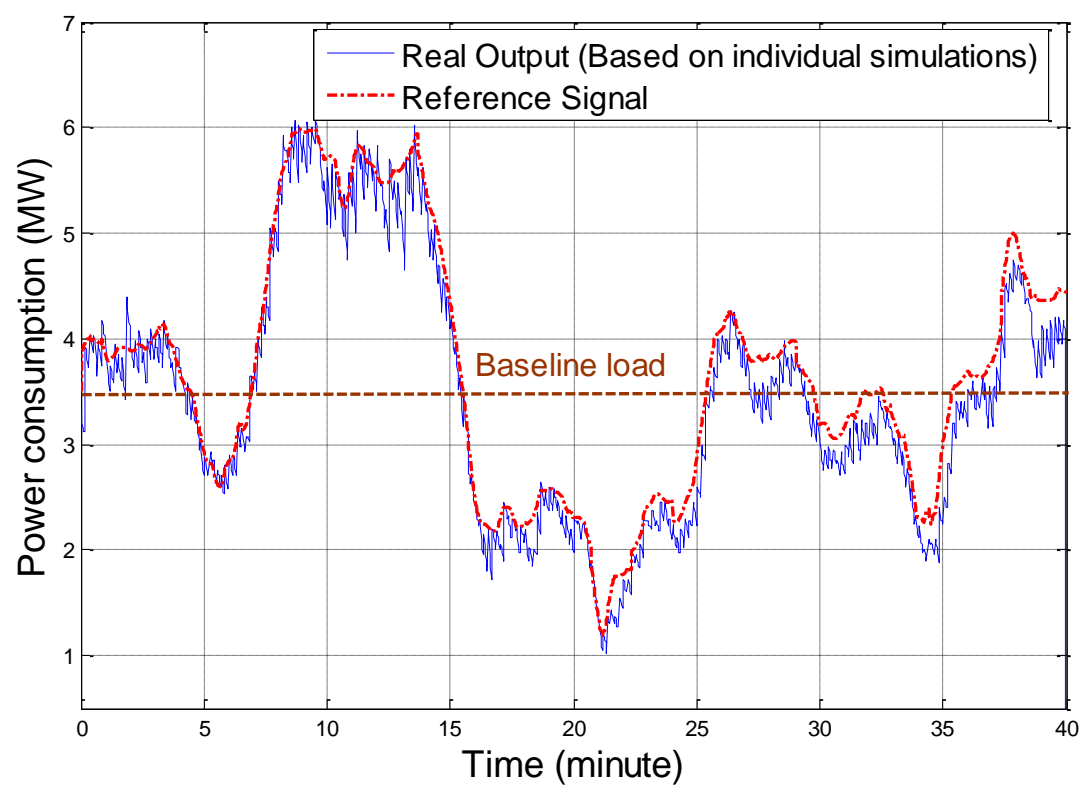

Figure 16 Performance of tracking the dynamic regulation test signal; the real output refers to the aggregated power output of $5000 \mathrm{HVAC}$ units, each of which has a 5-minute lock-out time

\subsection{Conclusions}

In this section, aggregated models were developed for a heterogeneous population of TCLs to accurately capture their collective behavior using demand response controls. The aggregated model includes statistical information of a heterogeneous population by utilizing a clustering technique. It also accounts for the dynamics of the solid mass of the buildings, which is a second order effect neglected in 
past work. The developed aggregated models are validated against simulations of thousands of detailed building models using GridLAB-D under both steady state and severe dynamic conditions caused by temperature setpoint changes. More details of this validation can be found in Appendix A. The simulation results show that the aggregated model reproduces the simulation results of thousands of individual loads, when both large and frequent changes in the temperature setpoint are applied to the population. The aggregated load model is also able to accurately reproduce the oscillations in the transient behavior of the population. Tradeoffs between accuracy and complexity of the aggregated model can be obtained by adjusting the means of representing the statistical information of the population. For capturing the steady state and the average transient behavior of demand response, a simplified representation can be used. On the other hand, for more detailed study of power grid transients when using demand response, one may need to use more detailed statistical representation to capture all of the transient behavior, increasing accuracy while decreasing speed. These appealing properties distinguish the proposed aggregate model from many other models in the literature, and make the proposed model a general framework of TCL aggregation for various demand response applications.

As part of the aggregated model, the lockout effect of the compressor was also considered in order to identify the number of HVAC units in the population that are not controllable, subject to the load control signal. This is one of the first times that the lockout effect is considered in the aggregate modeling of HVAC units. Using the developed aggregate model, a novel closed-loop load control strategy was designed for the population of HVAC units to track a prescribed demand curve while maintaining satisfactory end-use performance. Simulation results demonstrated the effectiveness of the proposed load control strategy. 


\subsection{Integrated transmission and distribution simulation platform}

The previous sections of this report outlined techniques and concepts for modeling smart grid assets in an aggregated fashion, especially HVAC units. Representing the loads on a distribution feeder as an aggregate model is useful, but so is the interaction with the larger transmission grid. This chapter will briefly discuss the details of the PowerWorld-GridLAB-D interface and its usage for integrating transmission and distribution powerflow.

\subsection{Background}

Traditional power system analysis often splits the power grid into two distinct levels: transmission and distribution. Transmission usually covers the larger power network, or bulk power network, and the dispersal of electricity over larger distances. This typically is from larger generators, such as nuclear plants or hydroelectric dams, to load centers like towns, cities, or large industrial complexes. Modeling typically stops at the substation or sub-transmission level and entire towns can be represented by a single load point. Detailed studies are conducted on conditions of the larger power system where generators are lost and transmission lines go out of service, but the end loads (typically representing cities or municipalities) are often left as fixed values.

Complimentary to the transmission modeling is distribution-level modeling. Distribution models typically begin where the transmission modeling ends. The entire bulk power network is often aggregated into a single connection point of the distribution model. From there, a detailed model of the individual distribution lines, connection points, and end-use loads is constructed. Detailed studies are conducted on voltage conditions, peak demand, and energy consumption. Transmission influences may be factored in (via varying voltage at the substation), but the focus is on the power getting from the substation to the end-use load.

With the advent of demand response and other technologies, end-use loads and distribution systems in general are becoming potential resources for mitigating transmission-level effects. Most simulation studies of the power system split into purely transmission or purely distribution studies, with little interaction between the two. An integrated tool is needed to examine the interaction of the traditional transmission system and rapidly evolving distribution system capabilities.

This particular project integrates a transmission-level powerflow solver and a distribution-level analysis package to investigate this interaction. With the interaction modeled, the impact of demand response on end-use devices and different operating strategies can be further explored. The effects of distribution-level controls and their impact on transmission-level operations can be examined. The integration of these resources and impact of transmission-level practices on end-use devices can also be more thoroughly examined. Overall, it provides a means for a more complete picture of the power system and a framework for investigating newer technologies and capabilities of the power system. 


\subsection{Software}

For this project, the integration of the transmission and distribution power system models builds off of two existing pieces of software. Both transmission and distribution solvers exist that are robust and mature and have evolved over decades of trial and error. Replicating one or the other was not considered effective. It was determined that integration of existing software would provide the requisite modeling flexibility, while capturing the years of experience and validation built into each tool. These two software tools communicate with one another to provide the basis for the integrated transmission and distribution environment.

\subsubsection{GridLAB-D}

The distribution solver selected for this task is GridLAB-D ${ }^{\mathrm{TM}}$. GridLAB-D is an open-source software package developed at the Pacific Northwest National Laboratory with funding from the Department of Energy, Office of Electricity Delivery and Energy Reliability [22]. The software provides a platform to analyze distribution systems all the way from the substation down to individual appliance behavior in the home.

As part of the distribution-level modeling, GridLAB-D includes detailed models for end-use power demand and energy consumption. The most notable is the equivalent thermal parameter model described in Section 2.1. With this thermal model, the cycling of individual HVAC units is tracked and their contributions to the distribution power system explicitly modeled. Other end-use devices, such as washers, dryers, and refrigerators, are modeled by similar multi-state models and post their power draw up to the powerflow model.

The distribution powerflow model within GridLAB-D models the electrical characteristics of the system. The explicit power requirements from the end-use devices are factored into an overall model. The overall model includes information on distribution lines, transformers, and any other electrical devices influencing a 1-second or longer powerflow solution on the system. Once constructed and solved, GridLAB-D provides information on voltage at the various points of the system, current flowing through lines, and overall power transfer on the system.

\subsubsection{PowerWorld Simulator}

The transmission-level solver selected was the PowerWorld Simulator 16 software [23]. PowerWorld Simulator is a commercial software package that includes many aspects of transmission modeling in a single, convenient package. Basic capabilities include powerflow solutions and contingency analysis, but capabilities can be expanded to include investigations of transient stability and optimal powerflow. The capabilities of the simulator allow the transmission system to be modeled from the generator all the way down to consolidated load representations of a populated area or industrial facility.

PowerWorld Simulator includes transmission-level models of various components of the system. This includes specific generator models, transmission lines, and even DC transmission systems. All of these items are combined with bus and load representations to model the transmission system in varying levels of detail. The end result is a model that allows the voltage, current, and power transmission quantities for various points of the system to be modeled. 


\subsection{Interface}

With the selection of the two software pieces, a method for integrating the two capabilities was required. PowerWorld Simulator will handle the transmission-level components all the way to an individual load connection point. From that point, GridLAB-D would provide a detailed model of the feeder network, all the way to the end use load. To handle communications between the load connections within PowerWorld Simulator and GridLAB-D, PowerWorld's SimAuto interface was utilized.

The PowerWorld SimAuto interface utilizes the Microsoft Component Object Model (COM) functionality to exchange information with external programs. GridLAB-D implements a basic COM interface to communicate with the PowerWorld Simulator software. Details of the implementation are available on the GridLAB-D Wiki page [24]. This implementation allows information between the two solvers to be exchanged. PowerWorld Simulator is slaved to GridLAB-D's clock, so all time progression in the analysis is coordinated from the GridLAB-D side.

Figure 17 shows a rough breakdown of how PowerWorld Simulator and GridLAB-D interface together. Individual feeders or connection points of the distribution feeders are connected via pw_load objects and substation objects. These two objects handle the communication and translation between the positive-sequence PowerWorld transmission solution and the three-phase unbalanced GridLAB-D solution. Convergence criteria are handled using two conditions. The transmission side monitors voltage changes at the pw_load interface, and the distribution side monitors changes in power consumption at the substation interface. The two solvers iteratively exchange information until proper convergence criteria are met. 


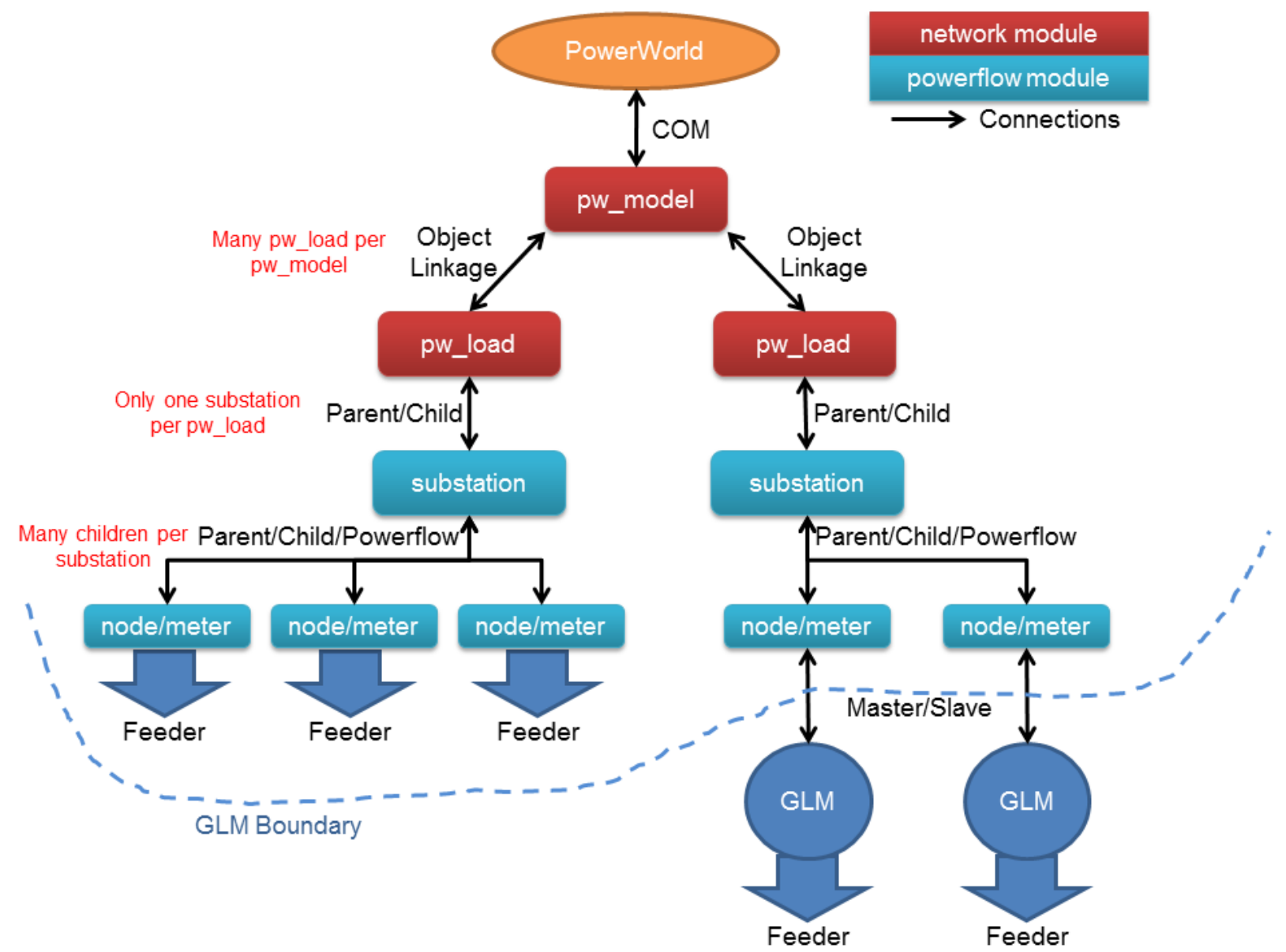

Figure 17 Rough structure for PowerWorld Simulator and GridLAB-D interface

\subsection{Validation}

Upon successfully interfacing the PowerWorld Simulator and GridLAB-D software packages, initial validation testing needed to occur. The validation of this section is aimed at assuring the solvers are communicating correctly, as well as ensuring the answers are consistent through trivial manipulations of the system.

Figure 18 shows the one-line diagram of the main system used to validate the PowerWorld Simulator and GridLAB-D interface. The small branch associated with Buses 10, 11, and 12 (highlighted in magenta) represents the adjustment point of the system. When validation began, Buses 10, 11, and 12 were all simulated within GridLAB-D using static loads (not time-varying), with the interface to PowerWorld at Bus 5. Under a second scenario, Buses 11 and 12 were simulated in GridLAB-D, whereas Bus 10 was now part of the PowerWorld model. This adjustment was repeated one more time so only Bus 12 was simulated inside GridLAB-D, with the rest simulated inside the PowerWorld Simulator. All simulations returned nearly identical answers, barring the convergence criteria and any errors associated with the unbalanced-to-positive sequence conversion between GridLAB-D and PowerWorld Simulator. 


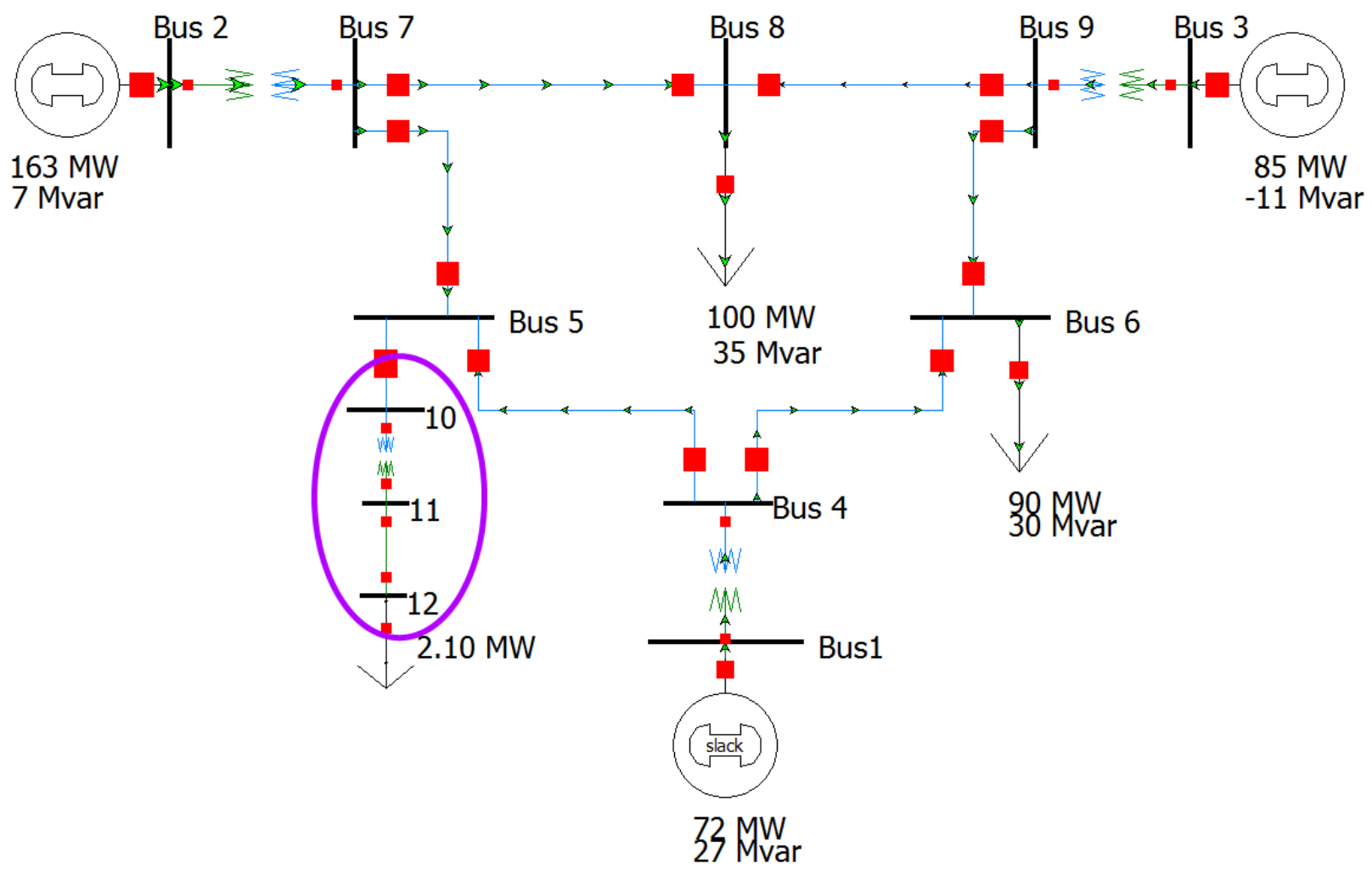

Figure 18 PowerWorld Simulator and GridLAB-D Validation Model - Magenta area represents the varying interface point

Further validation involved performing similar tests, but with time-varying load information. This examined whether GridLAB-D and PowerWorld Simulator could properly communicate and converge as the system changed with time. Figure 19 shows the time series plots the system of Figure 18, with Buses 10, 11, and 12 inside the GridLAB-D environment. As the load changed inside GridLAB-D, the representative load of Bus 5 inside PowerWorld changed in an identical manner. While this scenario is fairly simple, Figure 19 demonstrates that the communication between the two programs is working as intended, and simulations of integrated transmission and distribution models within this framework are properly handled. Without validating this result, using the integrated environment to examine the usefulness of demand-side resources and transmission-level impacts on the distribution system could not be fully evaluated. 




Figure 19 Time series plot of GridLAB-D and PowerWorld load values - Note PowerWorld represents a single-phase equivalent of the three-phase system

The details of the full validation are available in the integration tests included as part of the GridLAB-D network module auto-test folder [25]. The tests further explore the interfacing of the two programs, as well as the unit testing of individual components. All validation tests successfully passed with expected values, indicating the initial interface between GridLAB-D and PowerWorld Simulator is working correctly. This combination provides a simulation basis for simulating demand response controls on the population and how they impact the overall grid. 


\subsection{Integrated T\&D Control Use Case}

To explore the benefits of using an integrated transmission and distribution simulator, a use case was developed that utilizes the control methods discussed in Section 2.0. The use case explores how the direct load control signal developed in Section 2.4.2.1 could be applied for benefits at the transmission system. Specifically, in the event of a generator failure, how DR-enabled devices and a control signal developed through the closed-loop controllable aggregate model can be utilized for a spinning reserve call to reduce demand for 20 minutes while minimizing the synchronization and rebound at the end of the period.

For illustrative purposes, the transmission model was kept relatively simple - a modified three-node, two-area model from the PowerWorld sample files, as shown in Figure 20. The use case was modified from the original sample file by reducing the demand on Bus 2 so that when the Bus 2 generator is tripped, the line connecting Bus 1 and Bus 2 becomes overloaded, as shown in Figure 21. This overload represents the thermal limits on that line, but could easily represent transient stability or other limits. Note that the Bus 3 generator is capacity constrained at $150 \mathrm{MW}$, so the additional demand from the loss of the generator on Bus 2 must be met by the generator on Bus 1. Given the topology of the system, Branch 1-2 is the most likely to experience increased power flow.

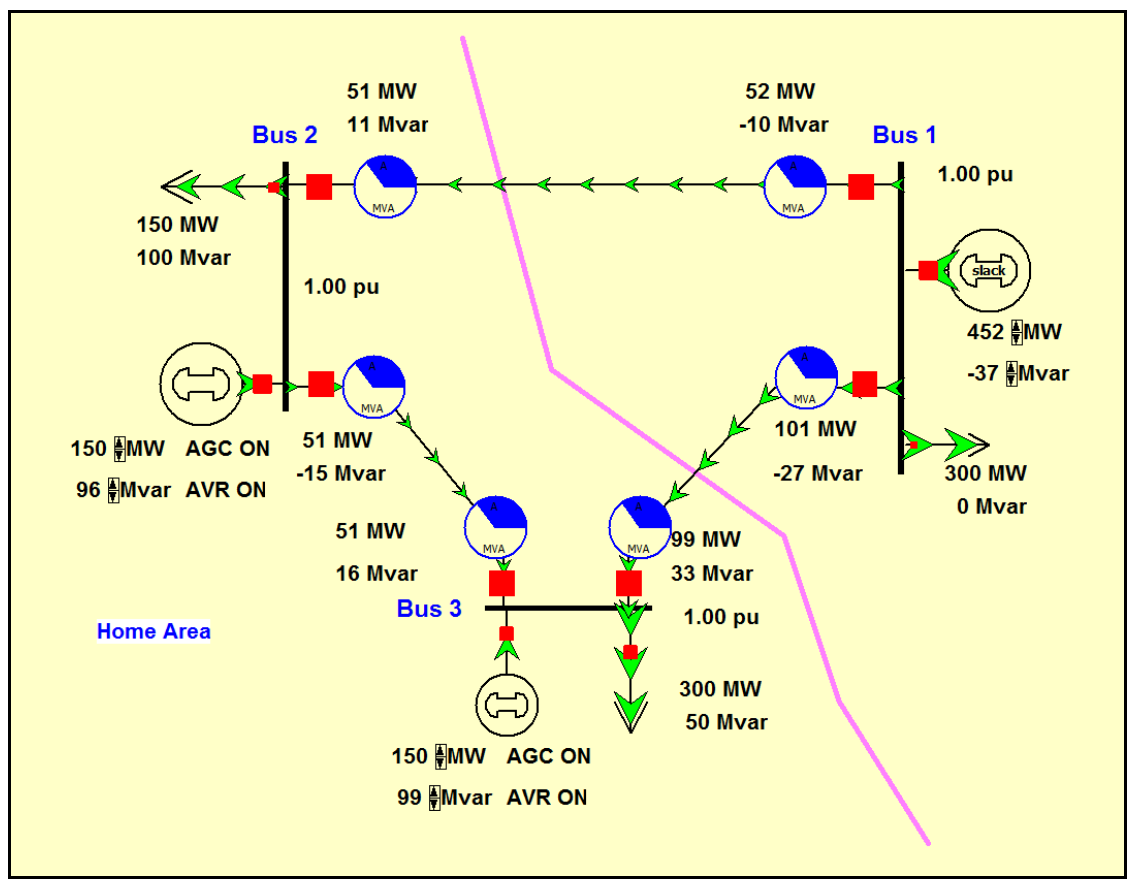

Figure 20 Modified PowerWorld model of three-node, two-area transmission system 


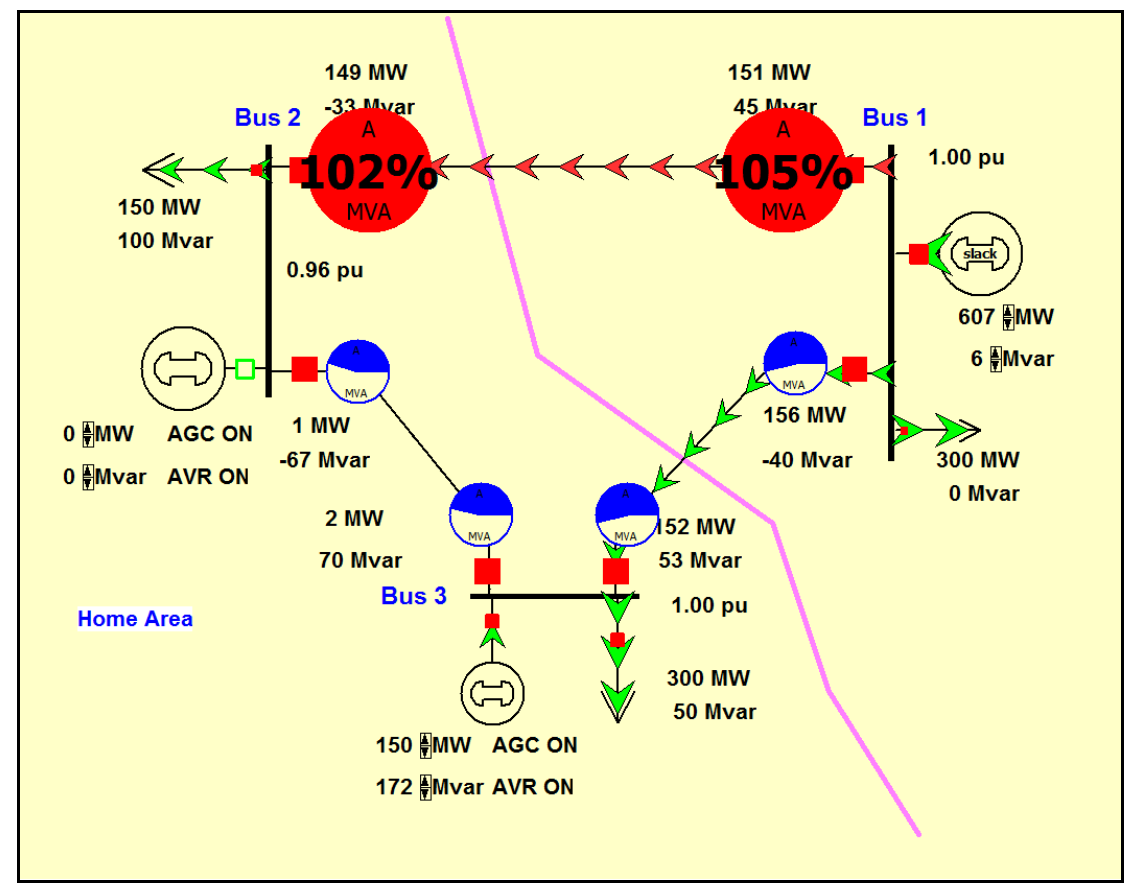

Figure 21 Modified PowerWorld model of three-node, two-area transmission system with Bus 2 generator tripped and Branch 1-2 overloaded

Typically, idle generation capacity at Bus 2, contracted for short term spinning reserves, would be called upon to ramp up production within 10-minutes of the loss of the generator and asked to operate for approximately 10-30 minutes until the supplemental reserves could be brought online. This is an inefficient use of resources, as the "spinning" generators are consuming fuel or operating at a reduced efficiency in order to reserve a certain amount of capacity in the event of a failure. Demand responseenabled devices are able to provide a similar balancing resource. Currently, some energy markets (for example, the New York Independent System Operator (NYISO) and the Midwest Independent System Operator (MISO)) allow large demand resources, such as industrial plants or large commercial buildings, to participate in spinning reserve markets. However, smaller, individual loads are typically not allowed to participate. By applying the aggregate control method described in Section 2.4.2.1, these small individually controlled loads can participate in the market in a controllable and reliable manner.

The 150 MW load at Bus 2 was replaced with a GridLAB-D distribution system model containing a static base load of $82 \mathrm{MW}$, representative distribution feeder power flow models, and 30,000 homes with DLC-enabled HVAC systems balanced relatively evenly across all three phases with a peak capacity of approximately $68 \mathrm{MW}$. At two hours into the simulation, the generator at Bus 2 was tripped, increasing line flows to the levels shown in Figure 21. The aggregate model responds by estimating the amount of available resource and reducing the HVAC demand by $16 \mathrm{MW}$ for 20 minutes, thereby reducing the line flows across Branch 1-2 to the levels shown in Figure 22 (the PowerWorld image is shown only for reference, as the GridLAB-D interface uses the PowerWorld GUI interface to communicate information; the PowerWorld GUI is unavailable during PowerWorld-GridLAB-D simulations). Figure 23 shows the three-hour time-series results for the total load at the Bus 2 substation. 


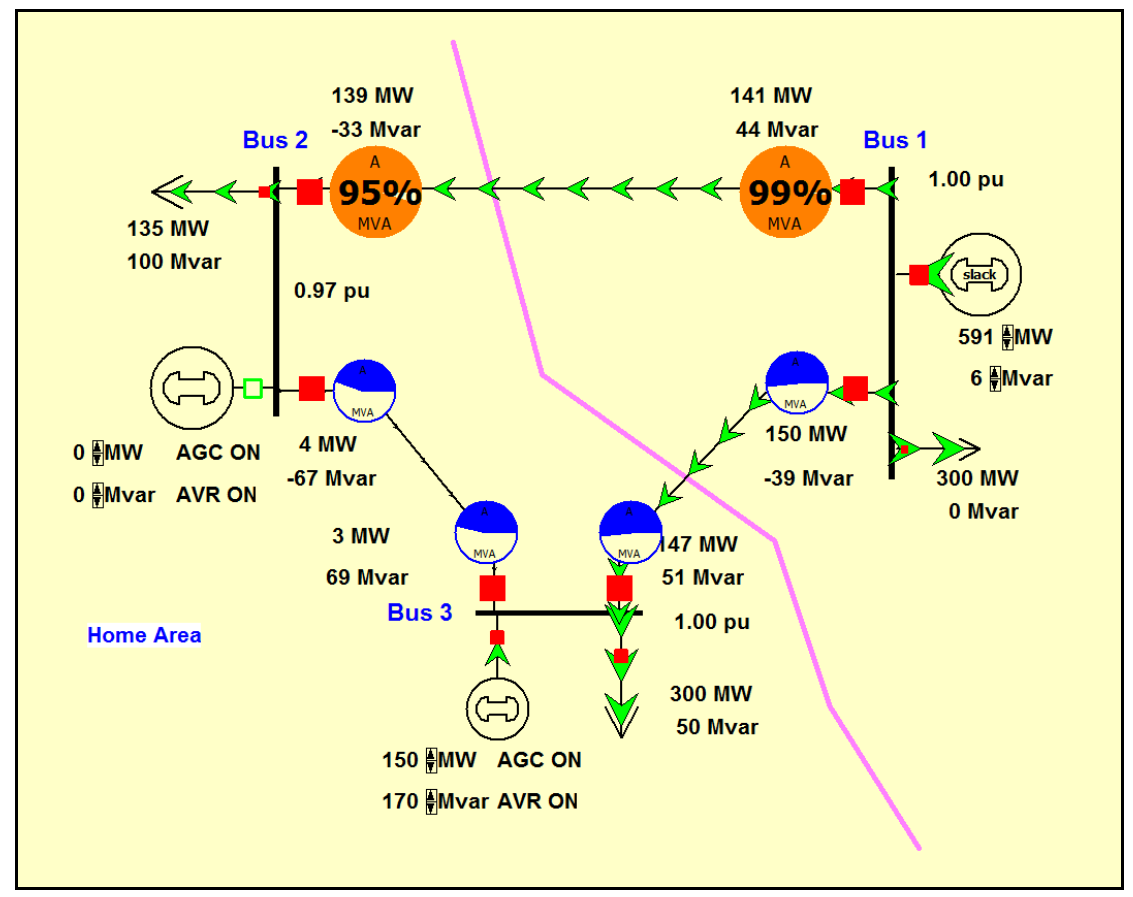

Figure 22 Modified PowerWorld model of three-node, two-area transmission system with Bus 2 generator tripped and Branch 1-2 congestion relieved by DLC-enabled HVACs at Bus 2

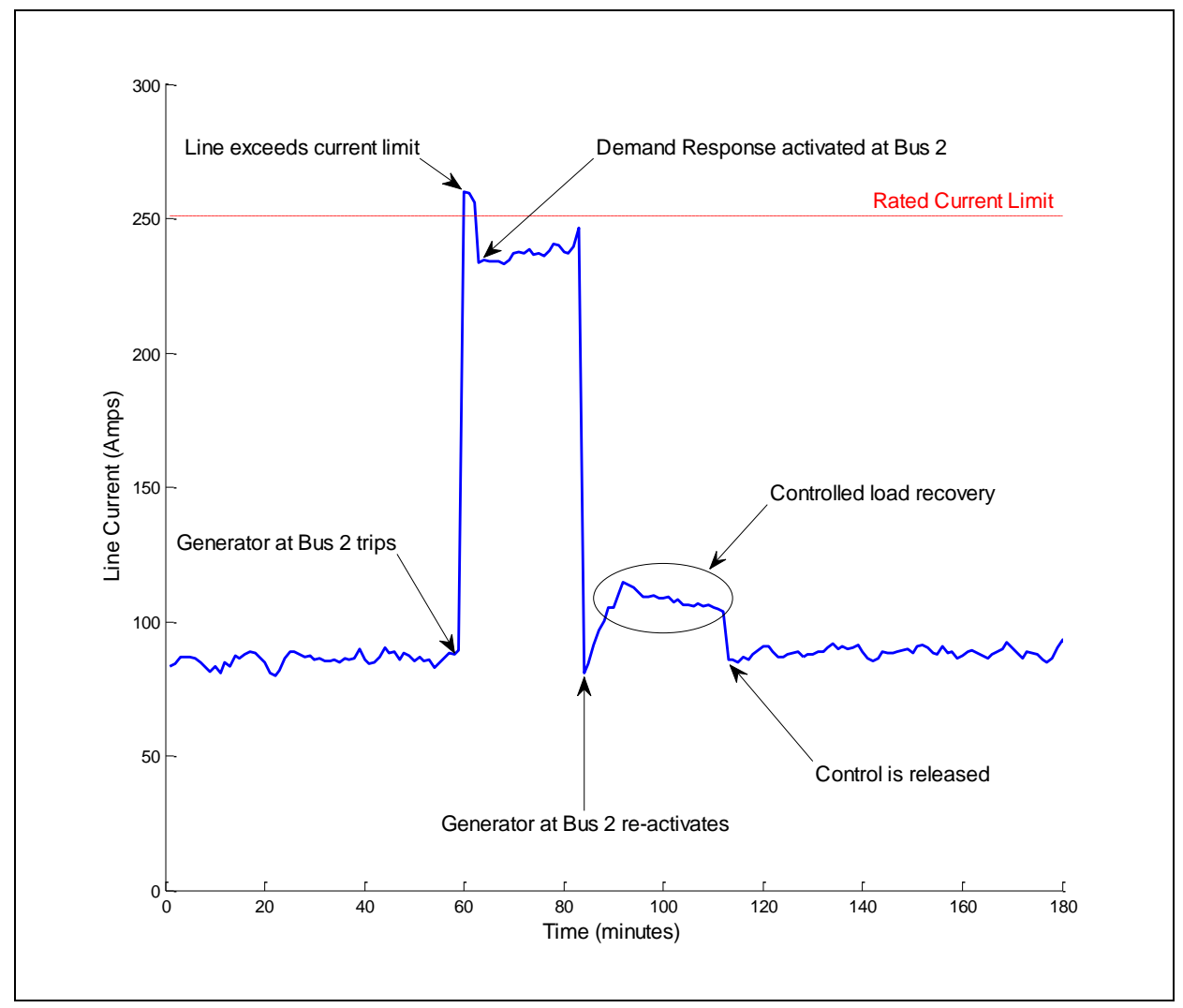

Figure 23 Time-series results for the total current flow through Branch 1-2 
The total load responds in a very predictable manner, reducing line flow through the branch by approximately 26 Amps for 20 minutes, until supplemental generation can come online. Once the supplemental generation comes online (or the original generator is restored), the control signal can also be used to manage the recovery, or rebound period, returning the "borrowed" energy back to the HVACs in a controlled manner during the next 30 minutes. This prevents large swings in the load immediately following the release back into normal operation, when devices may be synchronized by the control signal, and allows the supplemental generators to ramp up at a pre-described and manageable rate. This also prevents local equipment overloading and other damage to system components. At the end of 50 minutes, the devices are released back to normal operation. It should be noted that this is all done with a single centralized control signal that is broadcasted to all of the devices, and with no information coming from the devices back to the centralized control. This could be as simple as a radio broadcast, as there is no requirement for a communications feedback. The actual control is handled at the local level, relying on the devices to interpret and respond to the signal using local information, while responding in an aggregate fashion. This method also cycles through the customers who are responding (rather than directly commanding $25 \%$ "Off") and socializes the response by spreading the reduction to all of the devices in a coordinated fashion.

It should be acknowledged that this example is somewhat simplistic and ignores some of the ramping procedures that might be seen when transitioning from spinning to non-spinning reserves, and a number of other standard system controls. However, it illustrates the capabilities of the integration between the three tools. As the integrated transmission and distribution simulation tool is developed, use cases can be developed for a number of applications where the benefits accrued at the distribution level are focused on benefits at the transmission level (or vice versa). These benefits can be quantified and evaluated for potential applications. Applications can be envisioned, such as: wide area monitoring, protection, and control utilizing distributed resources; dynamic retail pricing for transmission congestion relief; and distributed resource participation in ancillary service markets, especially considering high intermittent generation penetration. New methods can be evaluated in a simulation environment that considers the impacts from generation to end-use load in a single simulation, and how the evolution of each through time affects the other. 


\subsection{Conclusions and Future Work}

Aggregated models were developed for a heterogeneous population of TCLs to accurately capture their collective behavior using demand response controls. The simulation results presented in Section 2.4 and Appendix A show that the aggregated model reproduces the simulation results of thousands of individual loads, when both large and frequent changes in the temperature setpoint are applied to the population. As part of the aggregated model, the lockout effect of the compressor was also considered in order to identify the number of HVAC units in the population that are not controllable, subject to the load control signal. Using the developed aggregate model, a novel closed-loop load control strategy was designed for the population of HVAC units to track a prescribed demand curve while maintaining satisfactory end-use performance. A number of direct load control signals (load shed, frequency regulation and tracking a particular power reference) were designed to evaluate the ability of the closedloop control strategy to manage a large distributed population of devices. The simulation results in Section 2.4, A.3.2 and A.3.3 indicate that, in general, the proposed closed-loop control strategy was able to accurately control the individual devices to the reference signal, with only a minor difference at the highest load period due to the reference signal saturating the devices.

To examine the interaction of the traditional transmission system and rapidly evolving distribution system capabilities, an integrated transmission and distribution tool was developed. In particular, the transmission-level solver was chosen to be the PowerWorld Simulator and GridLAB-D was chosen as the distribution-level analysis tool. This interface allowed the examination and evaluation of distributionlevel device controls on the larger power system. To explore the benefits of using an integrated transmission and distribution simulator, a use case was developed that explored how the direct load control signal developed in Section 2.4.2.1 could be applied for benefits at the transmission system. Specifically, in the event of a generator failure, how demand response enabled devices and the proposed control strategy can be utilized to reduce demand while minimizing the synchronization and rebound at the end of the period.

The integrated environment developed in this project allowed the load flow interactions between the bulk power system and end-use loads to be explicitly modeled down to approximately 1-second intervals. Future work will focus on expanding the capabilities of the integrated simulation environment for jointly modeling transient dynamics (less than 1 second). To fully examine the impacts distributionlevel demand response may have on the transmission-level grid, the recently developed dynamic capabilities of GridLAB-D will be integrated with the transient simulation capabilities in PowerWorld to model these grid-wide dynamic effects. With the integration of the PowerWorld transient simulation capability, the ability for demand response items, such as Grid Friendly Appliances ${ }^{\mathrm{TM}}$, to help maintain transmission system frequency and stability can be examined.

The aggregated model proposed in this work will be further developed to include effect of time varying parameters like outside temperature and solar irradiation. The closed-loop control strategies will also be further developed to include various practical issues, such as communication constraints, user privacy, telemetry requirements, operation rules, etc. Finally, the new control strategies will be validated on the integrated T\&D dynamic simulation environment. 


\section{References}

[1] S. Ihara and F.C. Schweppe, "Physically based modeling of cold load pickup", IEEE Transactions on Power Apparatus and Systems, 100 (9), pp. 4142-4150, Sept.1981.

[2] C.Y. Chong and A.S. Debs, "Statistical synthesis of power system functional load models", in Proc. of the 18th IEEE Conference on Decision and Control, 264-269, Dec. 1979.

[3] R. Malhame and C.Y. Chong, "Electric load model synthesis by diffusion approximation of a highorder hybrid-state stochastic system", IEEE Trans. on Automatic Control, 30 (9), pp. 854-860, Sept. 1985.

[4] D.S. Callaway, "Tapping the energy storage potential in electric loads to deliver load following and regulation with application to wind energy", Energy Conversion and Management, 50 (5), pp. 13891400, May 2009.

[5] D.S. Callaway and I. Hiskens, "Achieving Controllability of Electric Loads", Proc. of the IEEE, 99 (1), pp. 184-199, Jan. 2011.

[6] S. Banash and H. Fathy, "Modeling and Control Insights into Demand-side Energy Management through Setpoint Control of Thermostatic Loads", American Control Conference, June 2011.

[7] S. Koch, J. L. Mathieu, and D. S. Callaway, "Modeling and Control of Aggregated Heterogeneous Thermostatically Controlled Loads for Ancillary Services", $17^{\text {th }}$ Power system Computation Conference, Stockholm, Sweden, August 2011.

[8] K. Kalsi, F. Chassin and D. Chassin, "Aggregated Modeling of Thermostatic Loads in Demand Response: A Systems and Control Perspective", 50th IEEE Conference on Decision and Control, Dec. 2011.

[9] K. Kalsi, M. Elizondo, J. Fuller, S. Lu, and D. Chassin, "Development and Validation of Aggregated Models for Thermostatic Controlled Loads with Demand Response", 45th HICSS, Maui, Hawaii, January 2012.

[10] W. Zhang, K. Kalsi, J. Fuller, M. Elizondo, and D. Chassin, "Aggregate Model for Heterogeneous Thermostatically Controlled Loads with Demand Response," IEEE PES General Meeting, San Diego, CA, July 2012.

[11] W. Zhang, J. Lian, C. Chang, K. Kalsi and Y. Sun, "Reduced-Order Modeling of Aggregated Thermostatic Loads With Demand Response", 51st IEEE Conference on Decision and Control Conference, Maui, Hawaii, December, 2012.

[12] "GridLAB-D Residential Module User's Guild". Available: http://sourceforge.net/apps/mediawiki/gridlab-d/index.php?title= Residential_module_user\%27s_guide

[13] R. Sonderegger, "Dynamic Models of House Heating Based on Equivalent Thermal Parameters", Report PU/CES 57, Doctoral Dissertation 1978, Princeton University, Princeton, New Jersey.

[14] K. Subbarao, "Thermal Parameters for Single and Multizone Buildings and Their Determination from Performance Data", Solar Energy Research Institute, 1981, Golden, Colorado.

[15] R. T. Ruminsky. Compressor minimum off-time system, December 5 1978. US Patent 4,128,854.

[16] N. W. Wilson, B.S. Wagner and W.G. Colborne, "Equivalent Thermal Parameters for an Occupied Gas-Heated House", ASHRAE Transactions, 1985, vol. 91, part 2.

[17] Cristian Perfumo, Ernesto Kofman, Julio H. Braslavsky, and John K. Ward. Load management: Model-based control of aggregate power for populations of thermostatically controlled load. Energy Conversion and Management, 55:36-48, 2012.

[18] PJM manual 12: Balancing operations. available at http://www.pjm.com//media/documents/manuals/m12.ashx.

[19] PJM empirical analysis of demand response baseline methods. available at http://pjm.com/marketsand-operations/demandresponse $/ \%$ media/markets-ops/dsr/pjm-analysis-ofdrbaselinemethods-fullreport.ashx. 
[20] PJM markets \& operations. available at http://www.pjm.com/marketsand- operations/ancillaryservices/mkt-based-regulation.aspx.

[21] K.P. Schneider, J.C. Fuller, D.P. Chassin, "Multi-State Load Models for Distribution System Analysis," to be published IEEE Transactions on Power Systems, May 2011.

[22] GridLAB-D Contributors, GridLAB-D, [Online]. December, 2012. Available: http://www.gridlabd.org. Accessed December 7, 2012.

[23] PowerWorld Corporation, PowerWorld Simulator. [Online]. Available: http://www.powerworld.com/products/simulator/overview. Accessed December 7, 2012.

[24] GridLAB-D Contributors, "Spec:Transmission". [Online]. October 13, 2012. Available: https://sourceforge.net/apps/mediawiki/gridlab-d/index.php?title=Spec:Transmission. Accessed December 7, 2012.

[25] GridLAB-D Contributors, "Network Module Autotest Repository", [Online]. November 12, 2012. Available: http://gridlab-d.svn.sourceforge.net/viewvc/gridlab-d/trunk/network/autotest/. Accessed December 7, 2012. 


\section{Appendix A}

\section{Extensive Simulation Validation Results}


This section will describe the test cases used for validating the aggregate model via GridLAB-D simulations. This includes validation of the aggregate model in steady state and transient states, the implemented controls, and the controls in a closed loop simulation deriving information from the aggregate model and applied to the GridLAB-D model. It is understood that simulated results do not perfectly represent actual systems, but in the absence of quality "real life" data and an actual system to perform studies upon, the detailed, agent-based models provided by GridLAB-D are the closest available representation.

\section{A.1 Case 1: Basic model validation}

A GridLAB-D model containing 1000 diversified homes was created. These homes contained a distribution of construction parameters (R-values, floor area, setpoints, etc.) that were considered representative of U.S. building stock. The house models and weather applied to them were simplified from the standard GridLAB-D residential building models using the following assumptions:

1. Outside air temperature was held constant throughout the simulation.

2. Incident solar irradiance was held constant throughout the simulation.

3. Humidity was set to zero.

4. Oversizing factor of HVAC equipment was set to zero.

5. All HVACs were designed to use HEAT_PUMP mode, but AUXILIARY mode was deactivated.

6. Internal gains were set to a static value (i.e., not time-based) based upon the floor area.

7. Thermostat cycle times and lockout times were set to (near) zero, unless otherwise noted.

Time series measurements were captured at 1-second to 1-minute intervals, depending upon the requirements of the test case. The GridLAB-D simulations were run for a 24-hour period of time, where the first four hours were ignored to remove transient initialization errors in the GridLAB-D model.

The simulations were performed by two separate teams, one using the GridLAB-D simulation environment and one the aggregate model built in MATLAB. After the GridLAB-D simulations were run, all of the required input data fields (average of $U_{a}, H_{m}, C_{a}, C_{m}, Q_{i}, Q_{h \text {-rated }}, Q_{s}$, and $C O P_{\text {rated }}$ for the entire population) were provided to the aggregate modeling team for recreating the results. Time series information, including percentage of devices on versus off and total system load, from each of the models was then compared. While a large variety of test cases were developed, a sampling of the cases are shown here. Each of the following figures compares the number of HVAC units in the "On" state or the total power demand from each of the simulations, depending upon the application.

\section{A.1.1 Steady state}

These tests were designed to test whether the aggregate model achieves the same steady state performance as the GridLAB-D models at various outdoor temperatures, average household thermostat setpoints, and building construction types. In general, the steady state results of the aggregate model are quite comparable to the results seen by the GridLAB-D simulations. The only difference seen between the two models is in the random fluctuations observed in the agent-based model versus a very flat response in 
the aggregate model. This is due to the greater amount of diversity within the agent-based model, which contains 1000 diversified and individual building simulations.

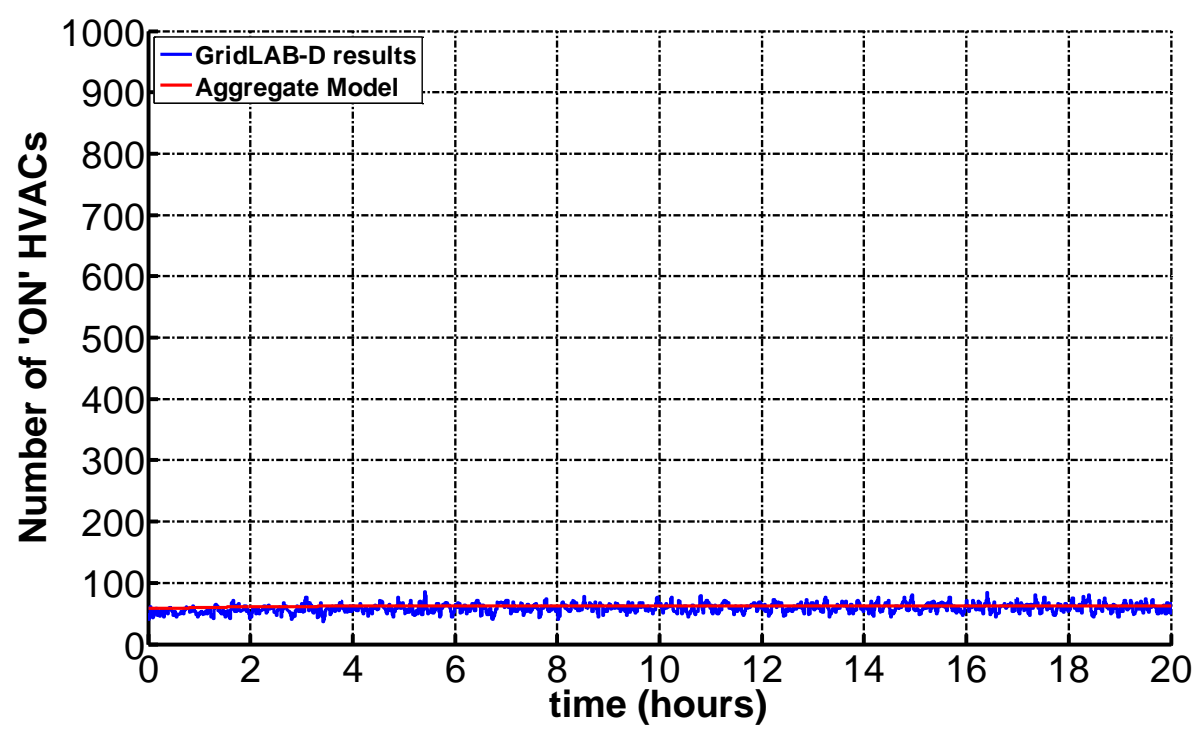

Figure 24 Outdoor temperature of $70^{\circ} \mathrm{F}$ and average cooling setpoint of $75^{\circ} \mathrm{F}$

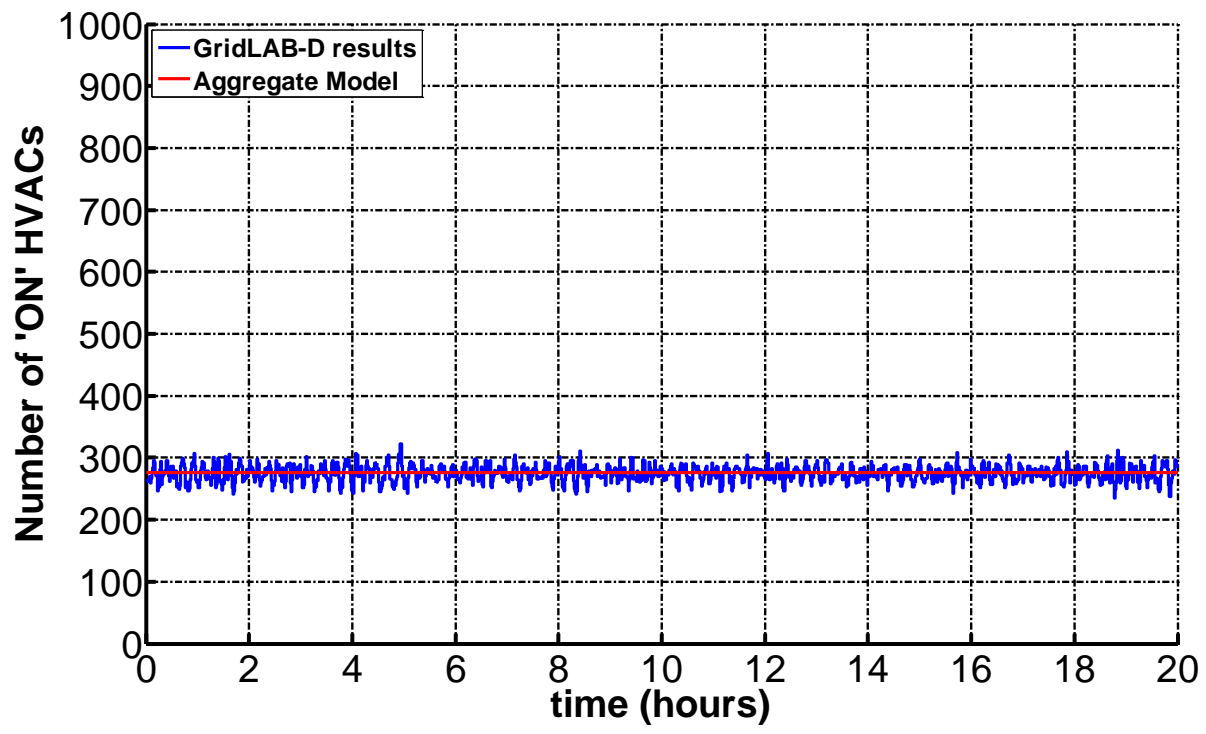

Figure 25 Outdoor temperature of $85^{\circ} \mathrm{F}$ and average cooling setpoint of $75^{\circ} \mathrm{F}$ 


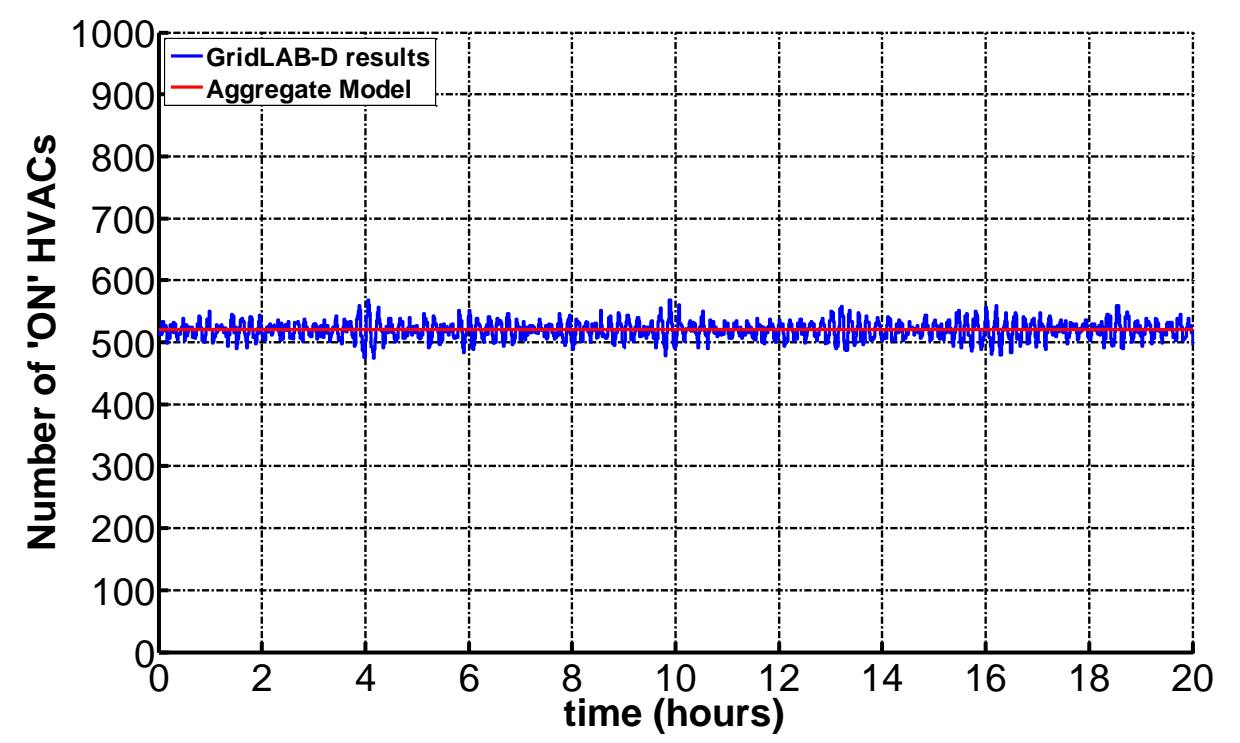

Figure 26 Outdoor temperature of $100^{\circ} \mathrm{F}$ and average cooling setpoint of $75^{\circ} \mathrm{F}$

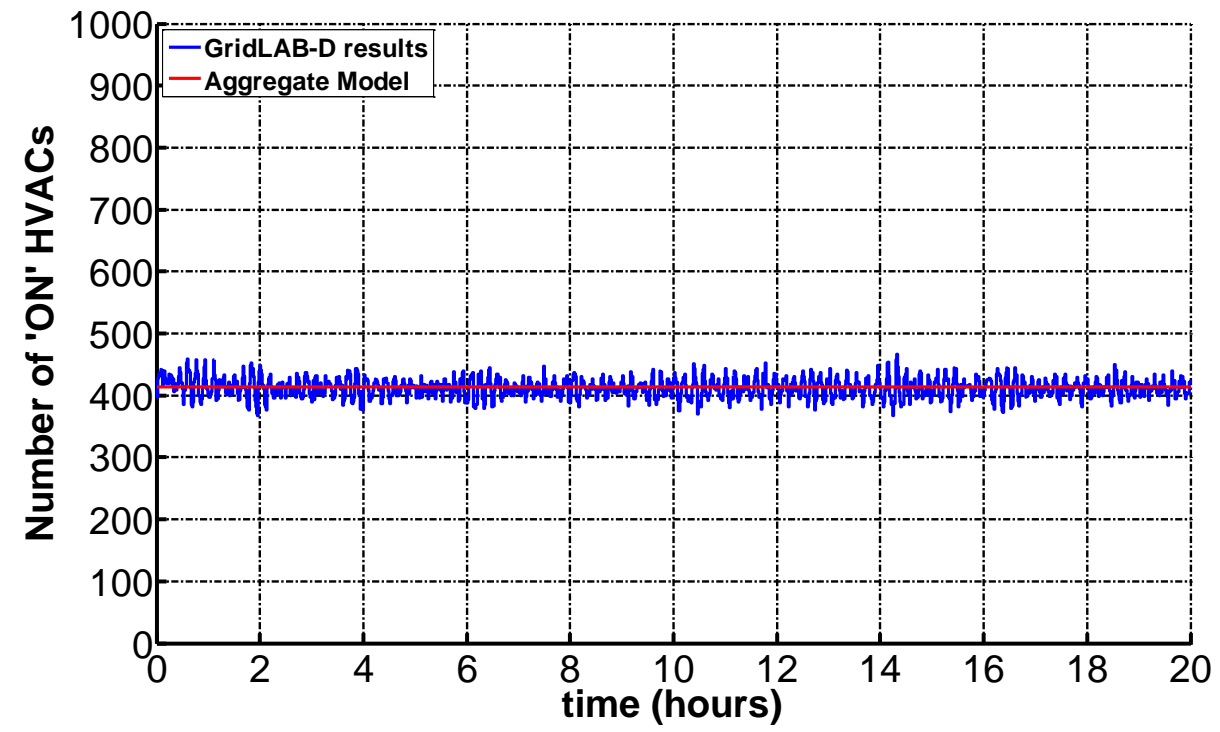

Figure 27 Outdoor temperature of $85^{\circ} \mathrm{F}$ and average cooling setpoint of $65^{\circ} \mathrm{F}$ 


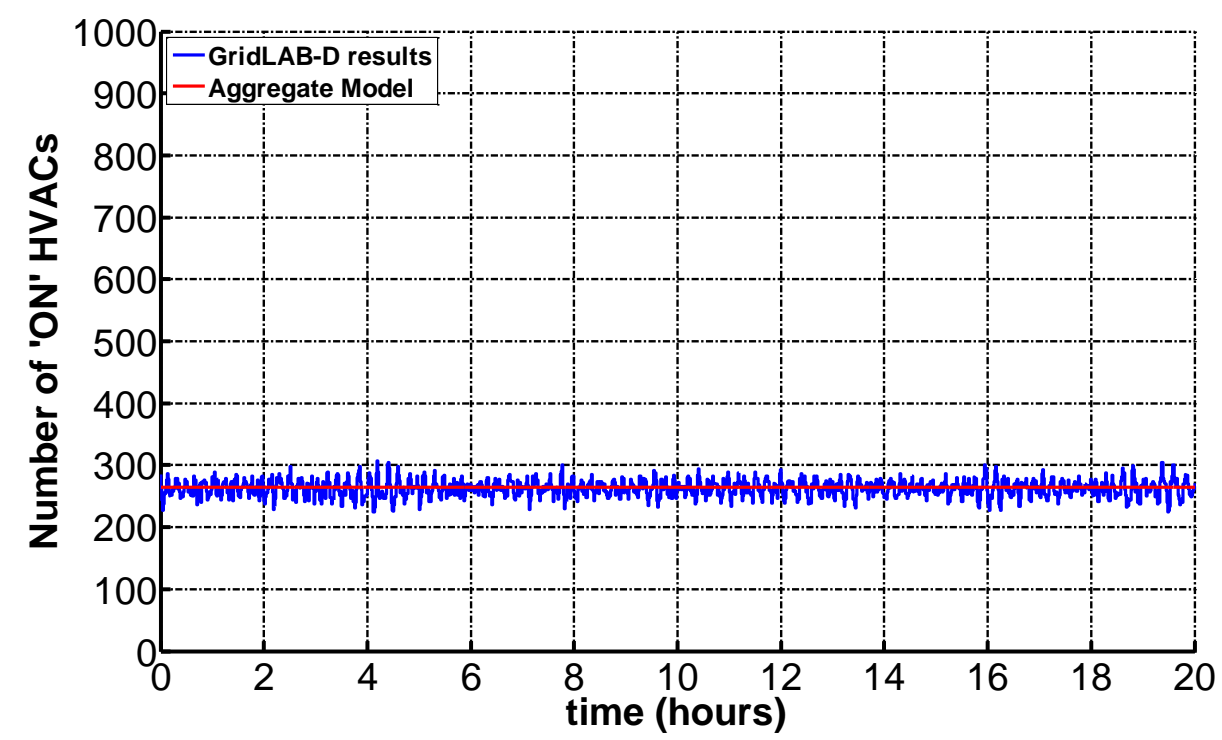

Figure 28 Outdoor temperature of $85^{\circ} \mathrm{F}$ and average cooling setpoint of $75^{\circ} \mathrm{F}$ using an alternate building design parameter distribution (e.g., different $U_{a}, H_{m}$, etc.)

\section{A.1.2 Change in cooling setpoint}

These tests were designed to evaluate the response of the two models to a population-wide change in the thermostat setpoint (e.g., all setpoints are shifted up by five degrees). This was used to test the aggregate models capability to represent both a variety of steady state conditions and the transient behavior between the steady states. The thermostat adjustment was applied in both directions (positive and negative adjustment) and for time intervals between ten minutes and six hours. In general, the results were in agreement (beyond the random fluctuations discussed previously) with a slight difference seen in the transient behavior, where the aggregate model generally returned at a slightly faster rate to steady state. 


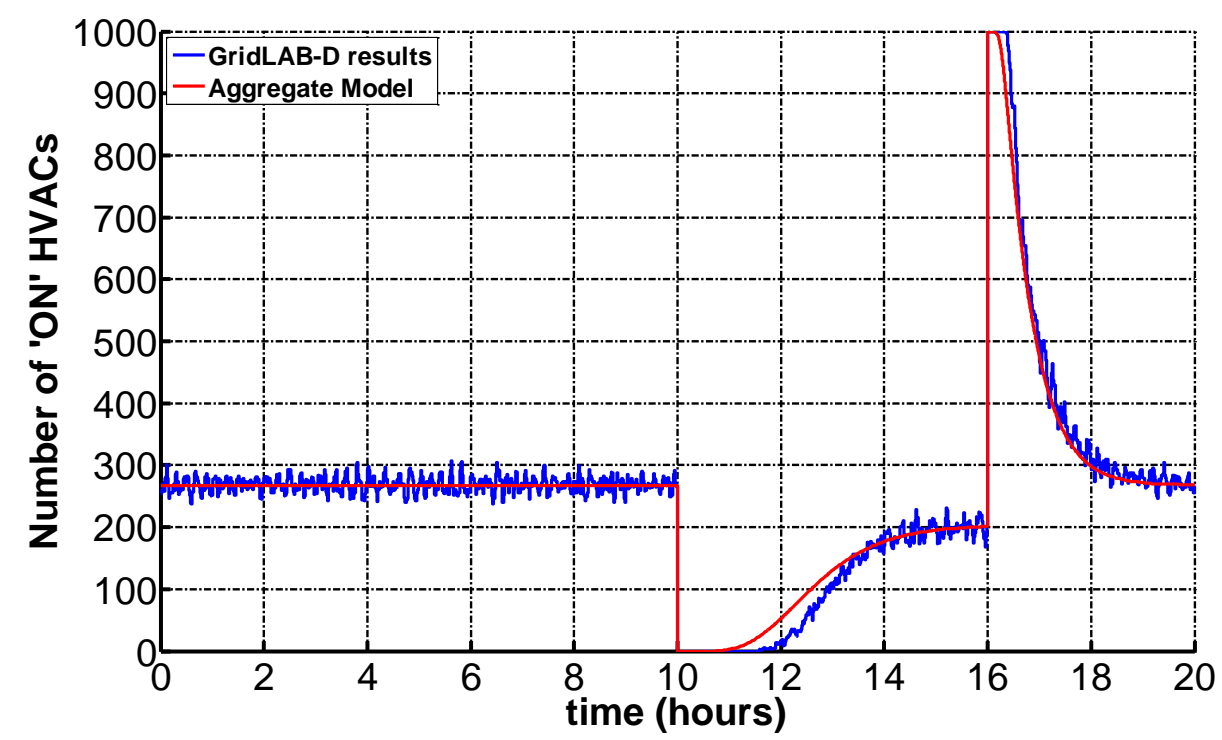

Figure 29 Outdoor temperature of $85^{\circ} \mathrm{F}$ and average cooling setpoint of $75^{\circ} \mathrm{F}$, applying a $+5^{\circ} \mathrm{F}$ delta for six hours to the cooling setpoint, then returning to normal

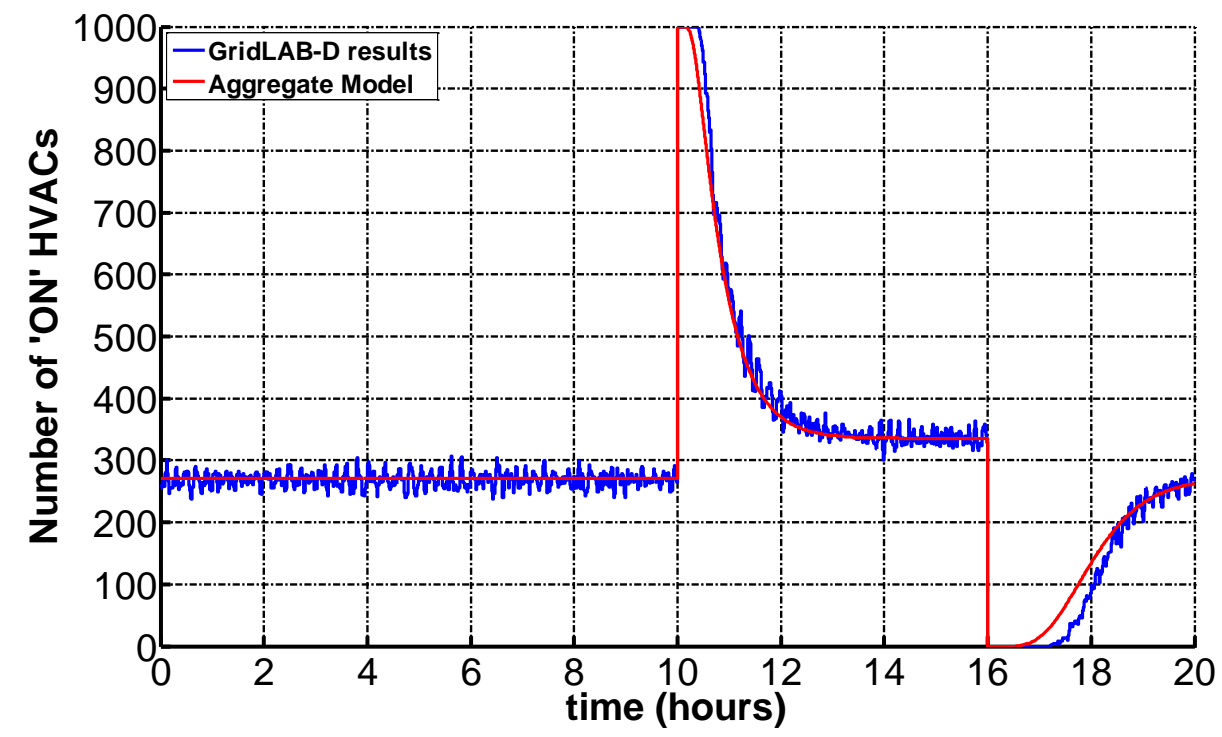

Figure 30 Outdoor temperature of $85^{\circ} \mathrm{F}$ and average cooling setpoint of $75^{\circ} \mathrm{F}$, applying a $-5^{\circ} \mathrm{F}$ delta for six hours to the cooling setpoint, then returning to normal 


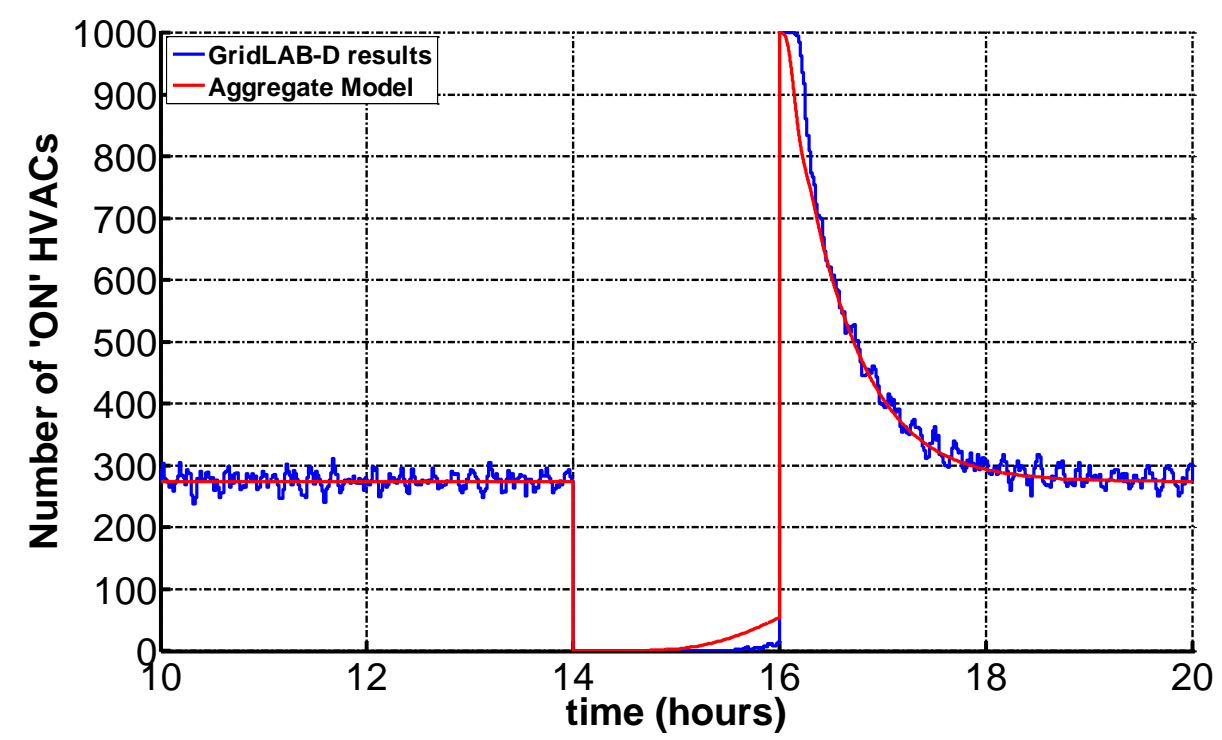

Figure 31 Outdoor temperature of $85^{\circ} \mathrm{F}$ and average cooling setpoint of $75^{\circ} \mathrm{F}$, applying a $+5^{\circ} \mathrm{F}$ delta for 2 hours to the cooling setpoint, then returning to normal, note that this includes a transition from one transient state to another without returning to steady state

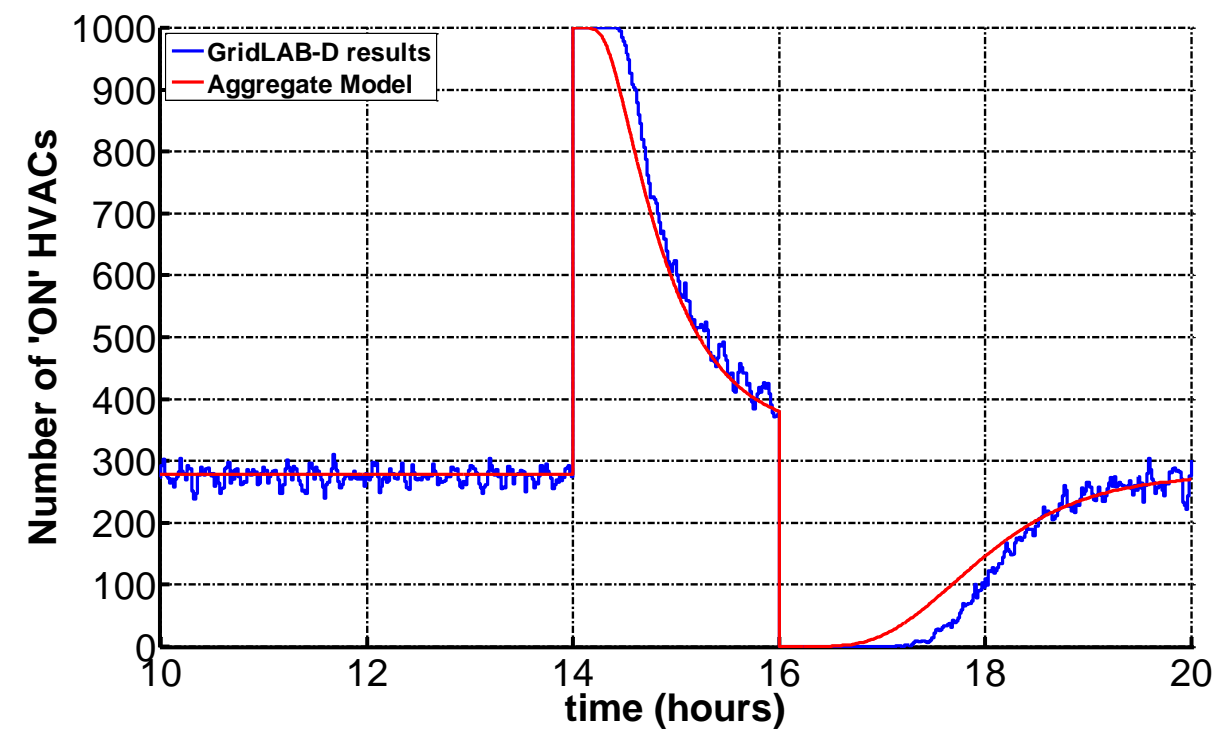

Figure 32 Outdoor temperature of $85^{\circ} \mathrm{F}$ and average cooling setpoint of $75^{\circ} \mathrm{F}$, applying a $-5^{\circ} \mathrm{F}$ delta for 2 hours to the cooling setpoint, then returning to normal, note that this includes a transition from one transient state to another without returning to steady state 


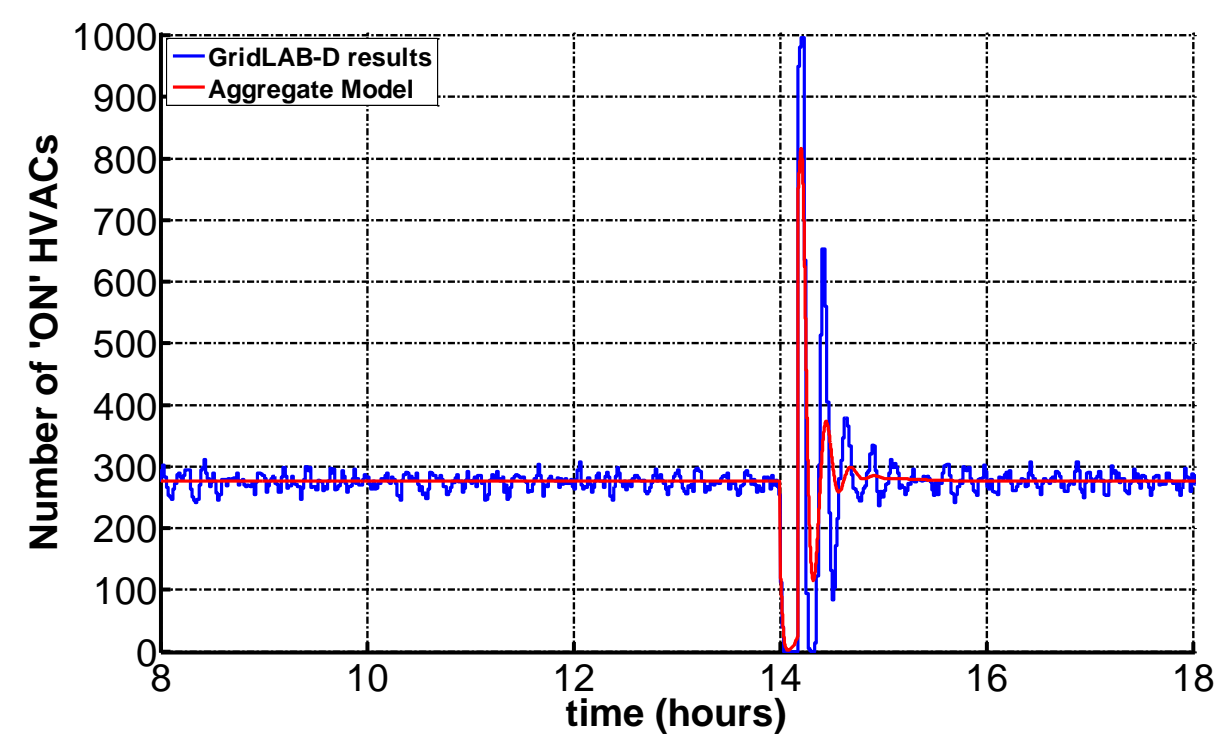

Figure 33 Outdoor temperature of $85^{\circ} \mathrm{F}$ and average cooling setpoint of $75^{\circ} \mathrm{F}$, applying $\mathrm{a}+1^{\circ} \mathrm{F}$ delta for 10 minutes to the cooling setpoint, then returning to normal

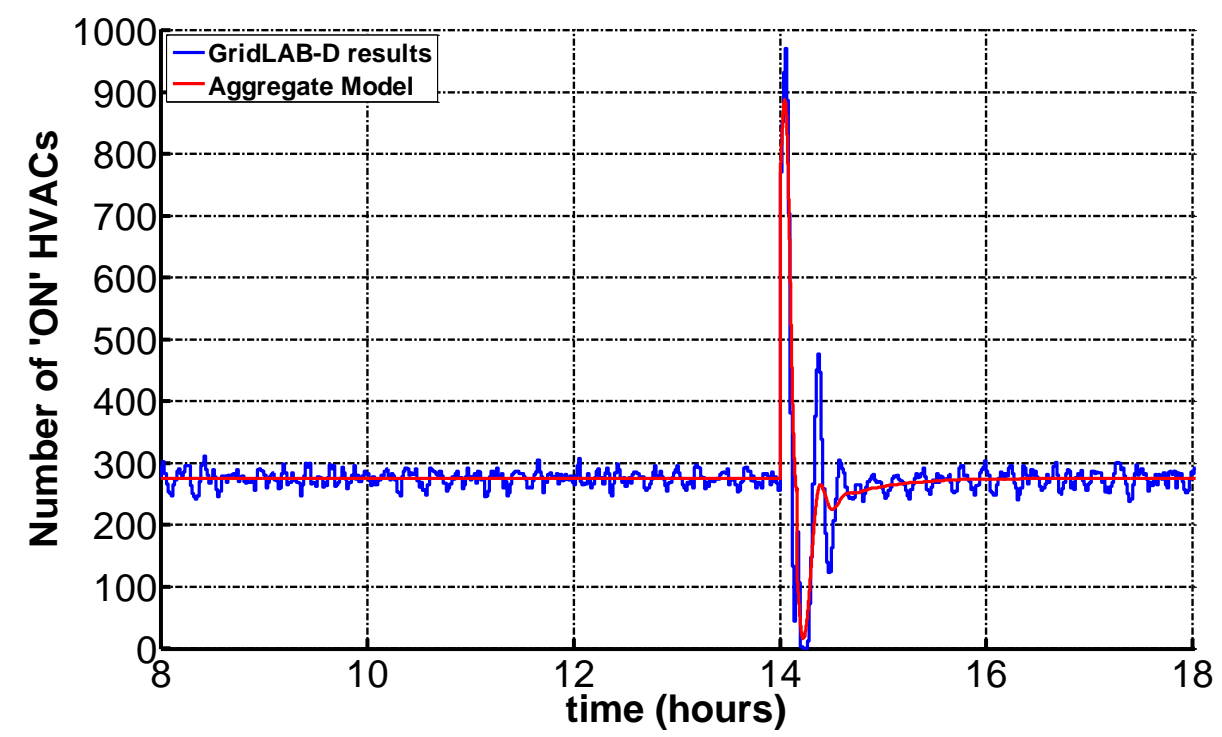

Figure 34 Outdoor temperature of $85^{\circ} \mathrm{F}$ and average cooling setpoint of $75^{\circ} \mathrm{F}$, applying a $-1^{\circ} \mathrm{F}$ delta for 10 minutes to the cooling setpoint, then returning to normal

\section{A.2 Case 2: Thermostat cycle time effects}

The models from Case 1 were reused to estimate the effects of thermostat lock out times. Thermostat cycle times were set to default in the GridLAB-D model (two minutes rather than zero minutes).

GridLAB-D represents thermostat lockout times in both the transition from "On" to "Off" (minimum run time) and "Off" to "On" (compressor relaxation time). These tests were designed to test the effects of ignoring these in the aggregate model. In general, it can be seen from the following figures that there is no 
noticeable difference in the comparisons between the two models on longer control time scales ( minutes to hours). In some instances (discussed in more detail in Case 3 ) that rely on very short cycling ( seconds) of the HVAC system, some minor differences can occur.

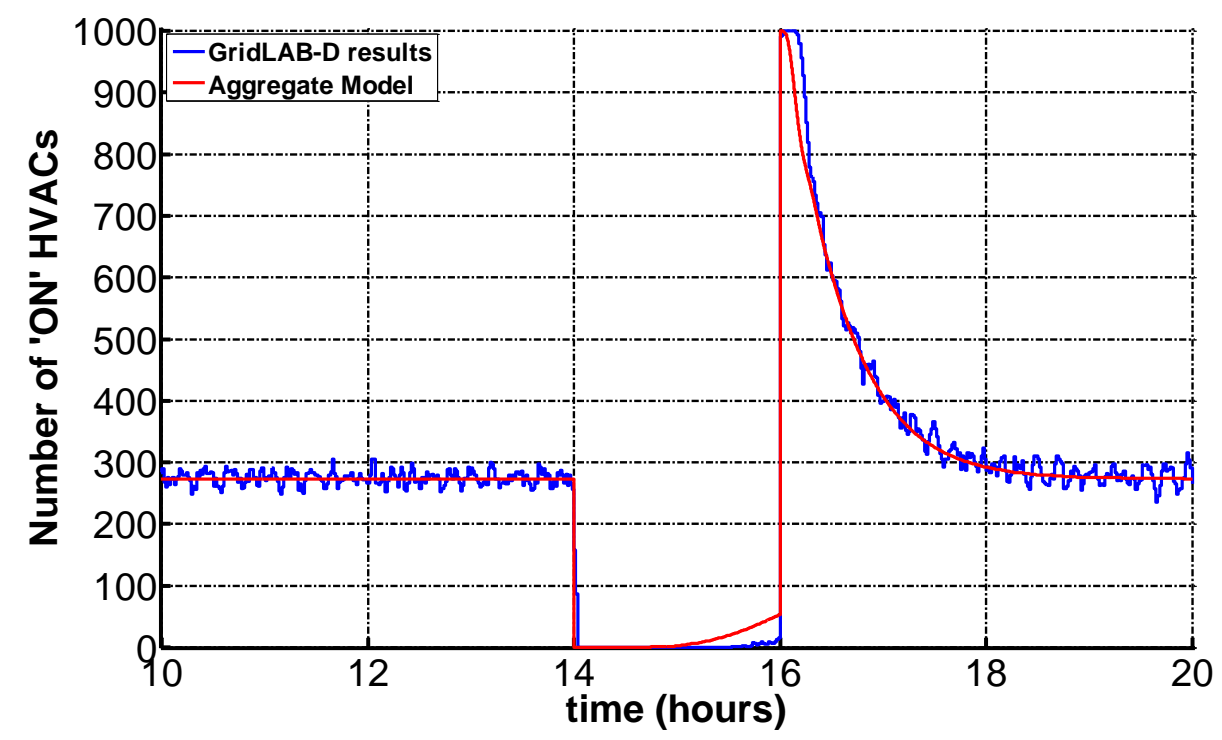

Figure 35 Outdoor temperature of $85^{\circ} \mathrm{F}$ and average cooling setpoint of $75^{\circ} \mathrm{F}$, applying a $+5^{\circ} \mathrm{F}$ delta for $\mathrm{t}$ hours to the cooling setpoint, then returning to normal, using GridLAB-D thermostat lockouts

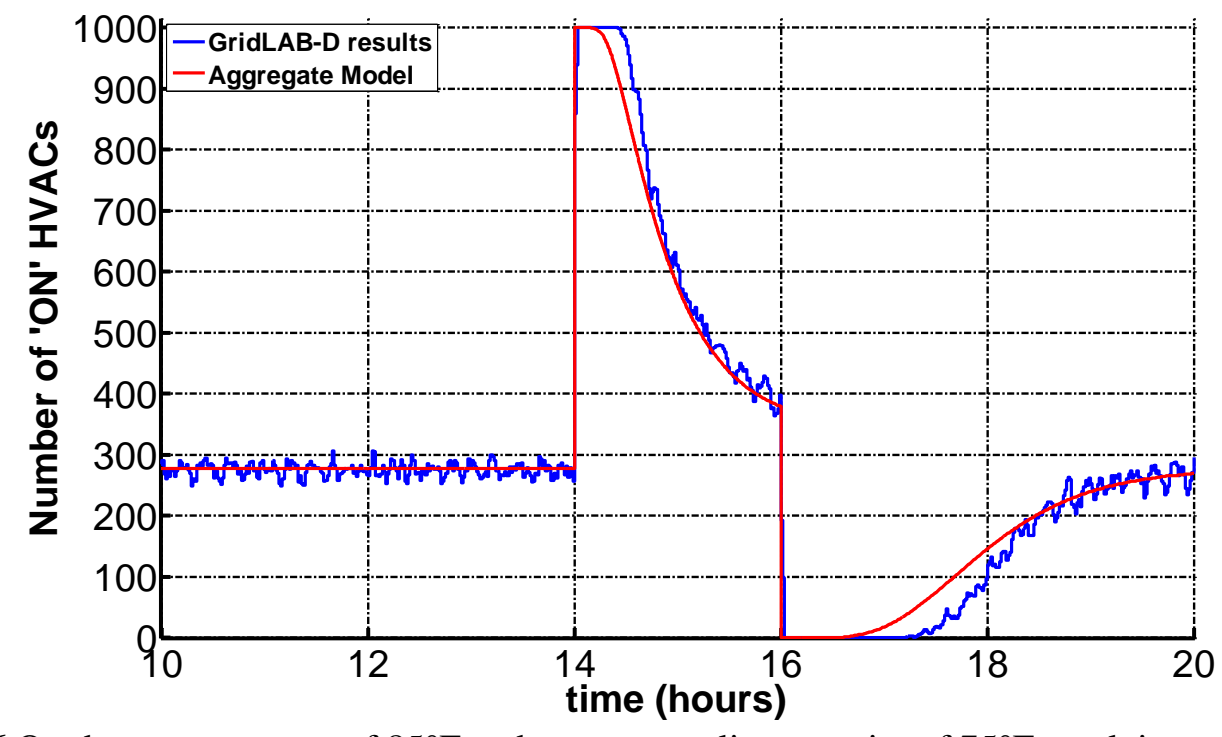

Figure 36 Outdoor temperature of $85^{\circ} \mathrm{F}$ and average cooling setpoint of $75^{\circ} \mathrm{F}$, applying a $-5^{\circ} \mathrm{F}$ delta for 2 hours to the cooling setpoint, then returning to normal, using GridLAB-D thermostat lockouts 


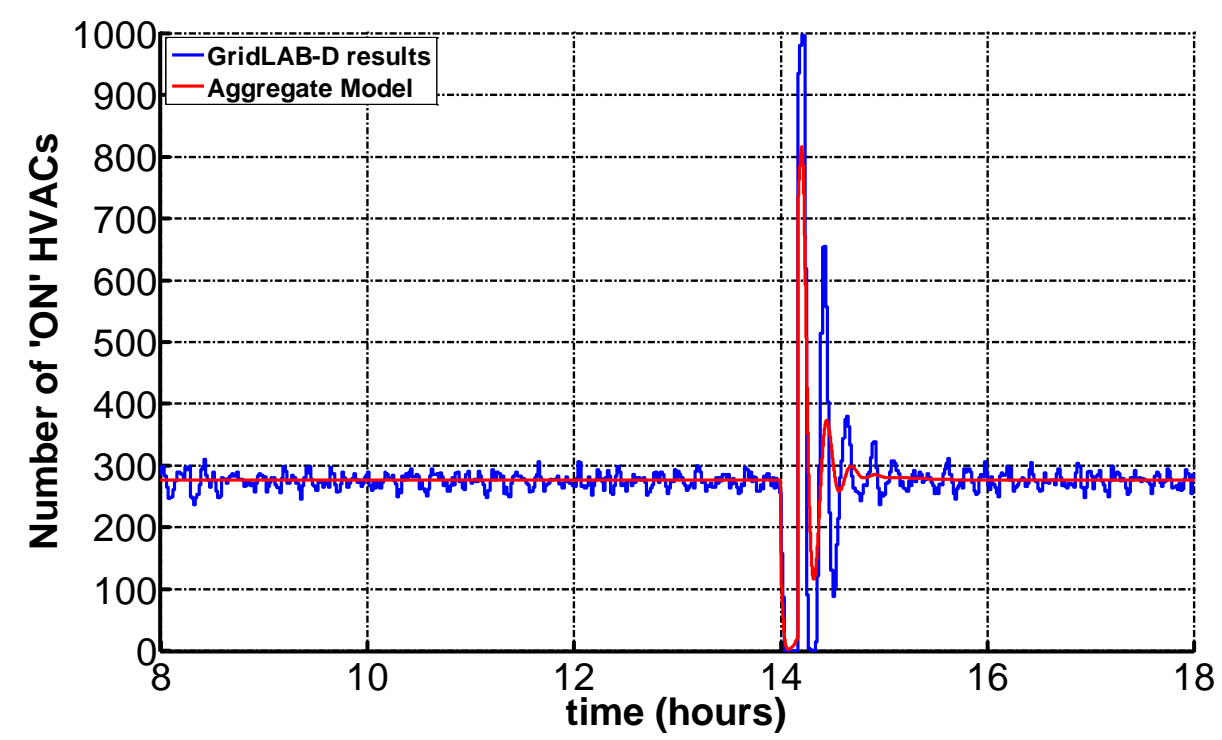

Figure 37 Outdoor temperature of $85^{\circ} \mathrm{F}$ and average cooling setpoint of $75^{\circ} \mathrm{F}$, applying a $+1^{\circ} \mathrm{F}$ delta for 10 minutes to the cooling setpoint, then returning to normal, using GridLAB-D thermostat lockouts

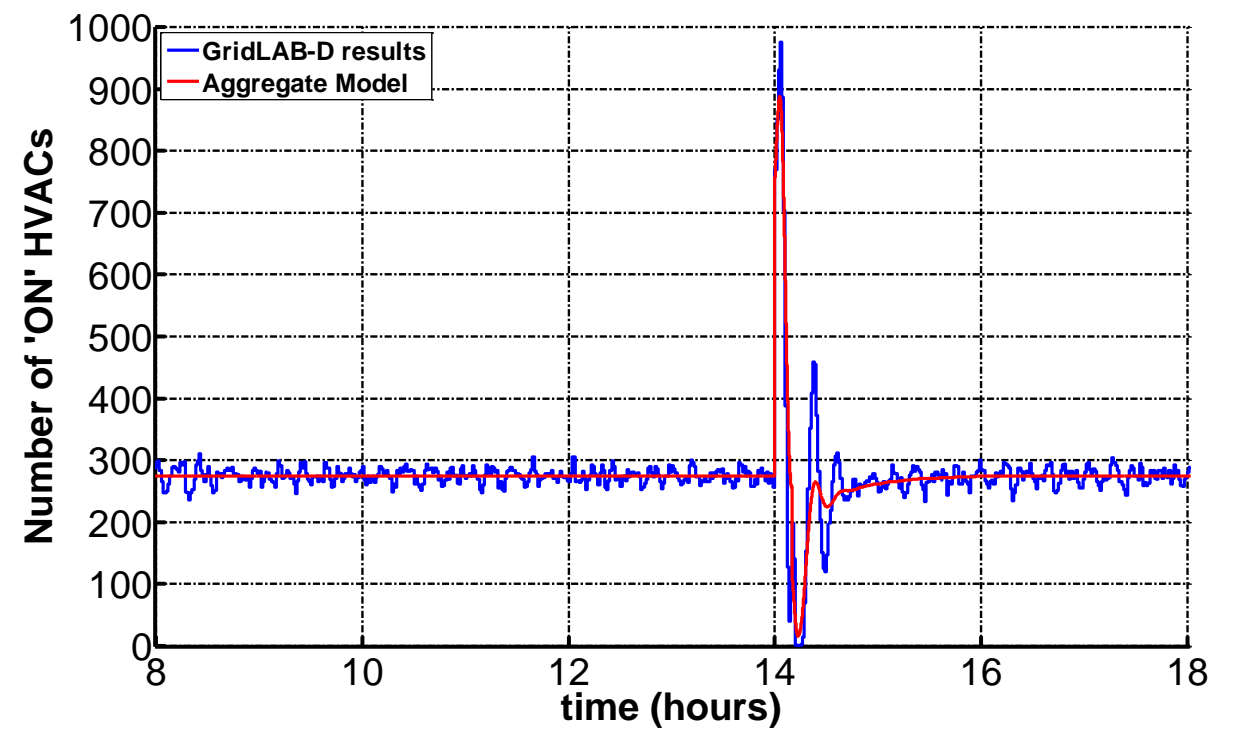

Figure 38 Outdoor temperature of $85^{\circ} \mathrm{F}$ and average cooling setpoint of $75^{\circ} \mathrm{F}$, applying a $-1^{\circ} \mathrm{F}$ delta for 10 minutes to the cooling setpoint, then returning to normal, using GridLAB-D thermostat lockouts

\section{A.3 Case 3: Direct Load Control}

To test the ability of the model to control devices in an "actual" system, the aggregate model control system developed in MATLAB was run in parallel with the GridLAB-D model. The control signal, $\alpha$, produced by the aggregate model was broadcasted to the individual devices within the GridLAB-D environment via a global control signal. The local control devices interpreted the signal as described in Section 2.4.2.1 so that the entire population would follow a reference signal. Some cases were tested via 
an open-loop control signal, while others utilized closed-loop control to correct for system inconsistencies. In the following figures, comparisons were made between the reference signal (in closedloop control), the aggregate model results, and the GridLAB-D results - in some cases this will be a measurement of load at the substation, in others it will be the number of cooling units on. The model from Cases 1 and 2 was again reused, with a cooling setpoint of $75^{\circ} \mathrm{F}$ and the same building construction parameters. Thermostat lockout times were used in some, but not in others. A number of control signals were tested to evaluate the ability of the reduced order control model to predict the requisite control signal for a distributed population of devices.

\section{A.3.1 Load shed signal}

These tests are designed to evaluate a control signal that toggles a certain percentage of the population that are in the "On" state to the "Off" state (i.e., reduce demand by a given percentage of appliances that are currently "On"). The population of devices are "held down" for a given amount of time then released back to normal operation. Note that the reduction is not sustained by the same devices that initiated the reduction, but rather the control signal sustains the reduction by short cycling the devices. This type of control signal might be used for a variety of short ( $\sim$ seconds) to long ( hours) reductions for meeting frequency regulation ( $\sim$ seconds) to spinning reserve requirements $(\sim 10-20$ minutes) to capacity management ( $\sim$ minutes to hours). In general, the aggregate model responds in a similar manner to the GridLAB-D model, in terms of the reduction and the envelope of the release period. However, it can be seen in a number of cases (notably Figure 43, Figure 45, and Figure 47) that the aggregate model overestimates the rebound. This is mainly due to the model reduction used in this validation test - only one cluster was used. This reduces the model accuracy, mainly in the transient time periods, but improves model performance. Additionally, in most of the cases the envelope of the aggregate model ring-down to steady state is less than the GridLAB-D model. This is a consequence of the decreased diversity within the aggregate model.

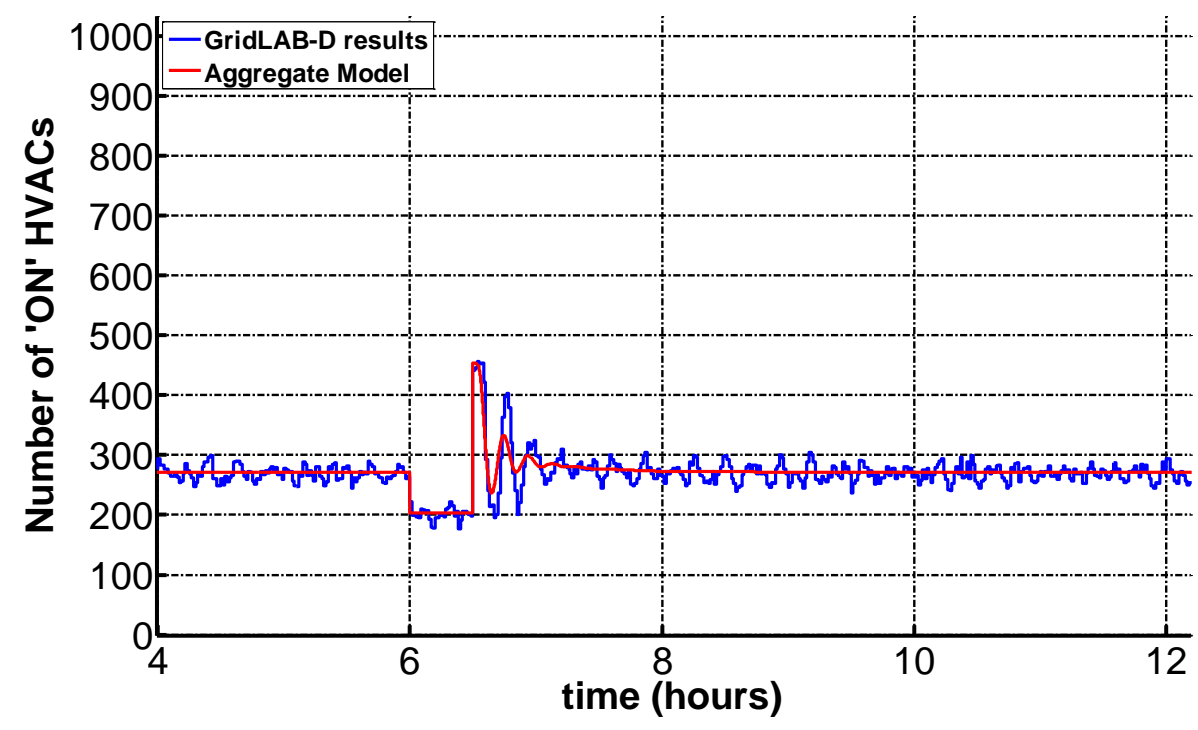

Figure 39 Outdoor temperature of $85^{\circ} \mathrm{F}$, lockout times set to (near) zero, and a $25 \%$ reduction in load that releases back to normal operation after 30 minutes 


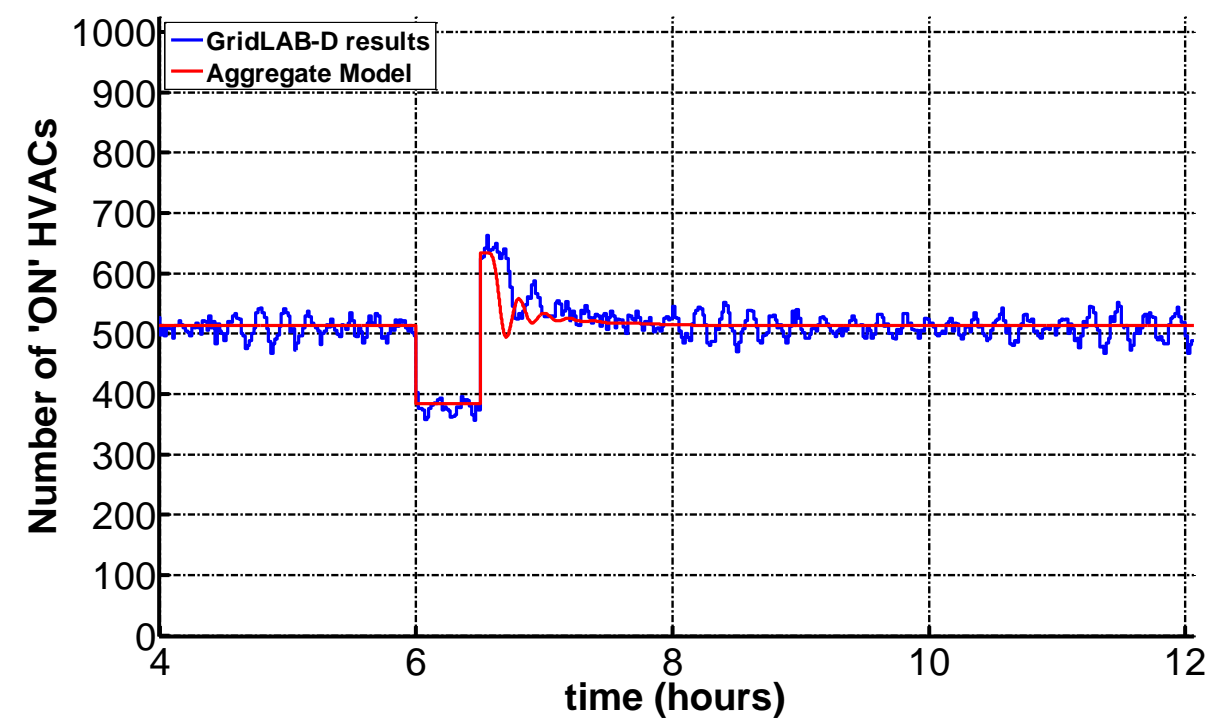

Figure 40 Outdoor temperature of $100^{\circ} \mathrm{F}$, lockout times set to (near) zero, and a $25 \%$ reduction in load that releases back to normal operation after 30 minutes

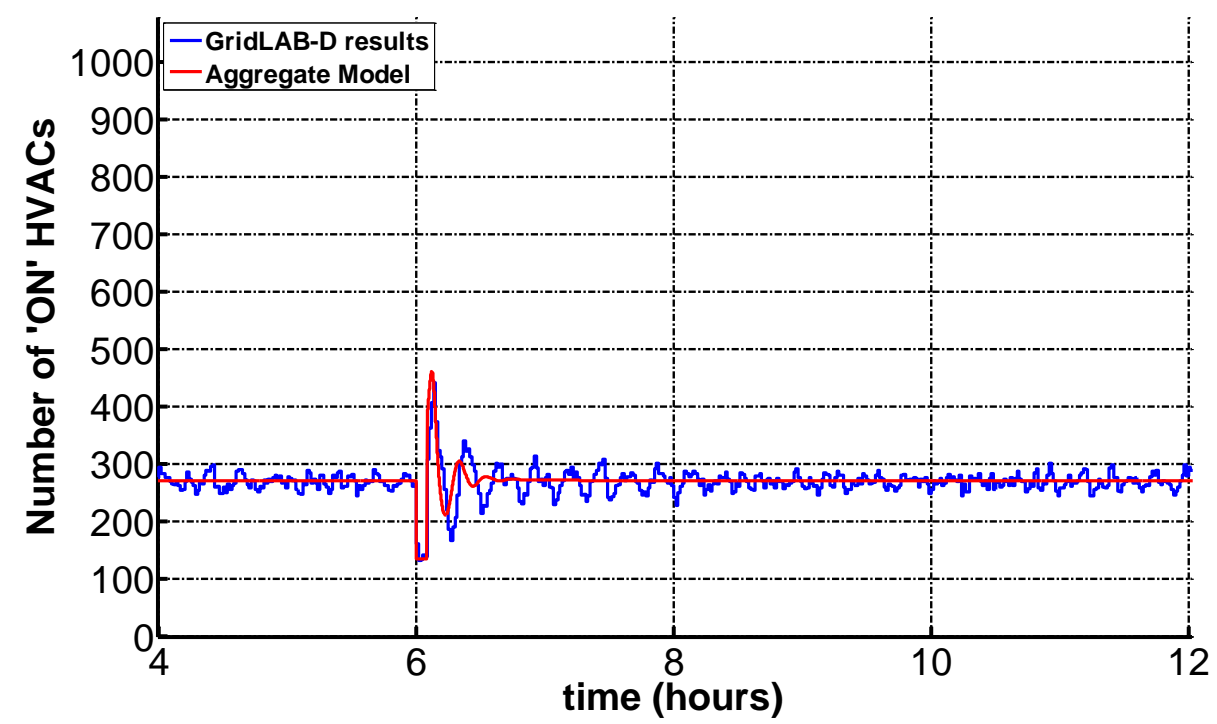

Figure 41 Outdoor temperature of $85^{\circ} \mathrm{F}$, lockout times set to (near) zero, and a $50 \%$ reduction in load that releases back to normal operation after 5 minutes 


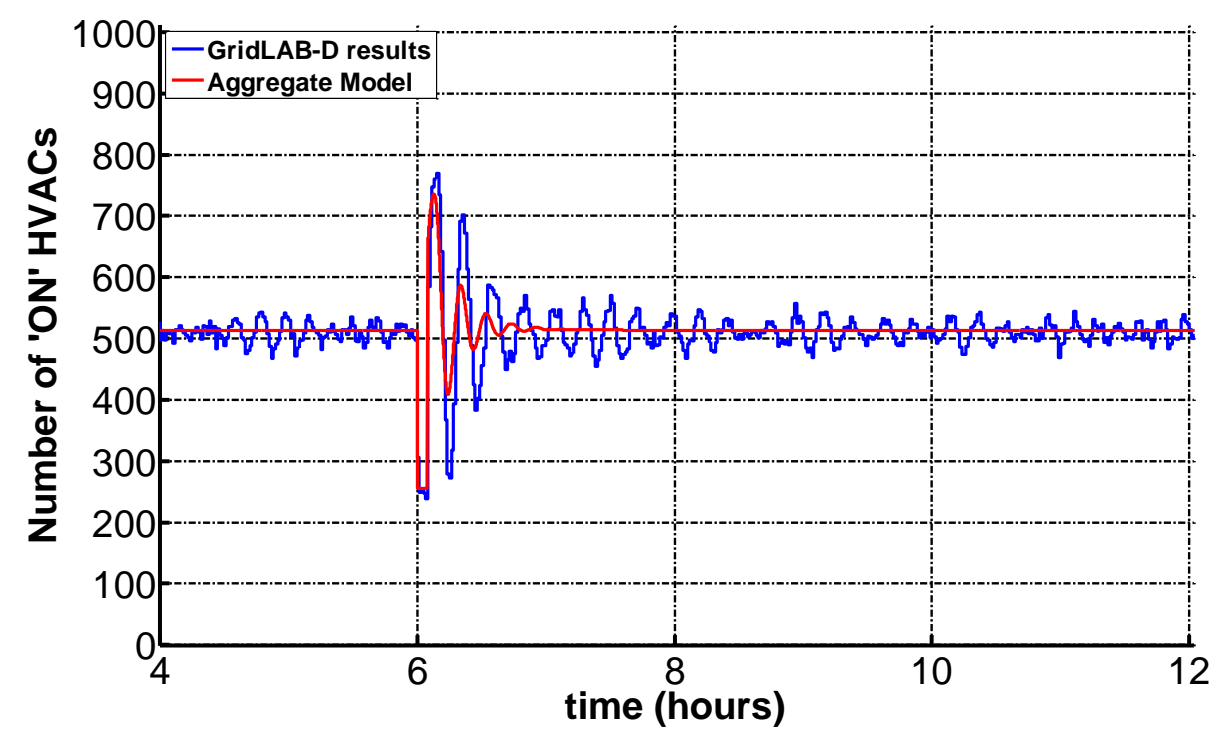

Figure 42 Outdoor temperature of $100^{\circ} \mathrm{F}$, lockout times set to (near) zero, and a $50 \%$ reduction in load that releases back to normal operation after 5 minutes

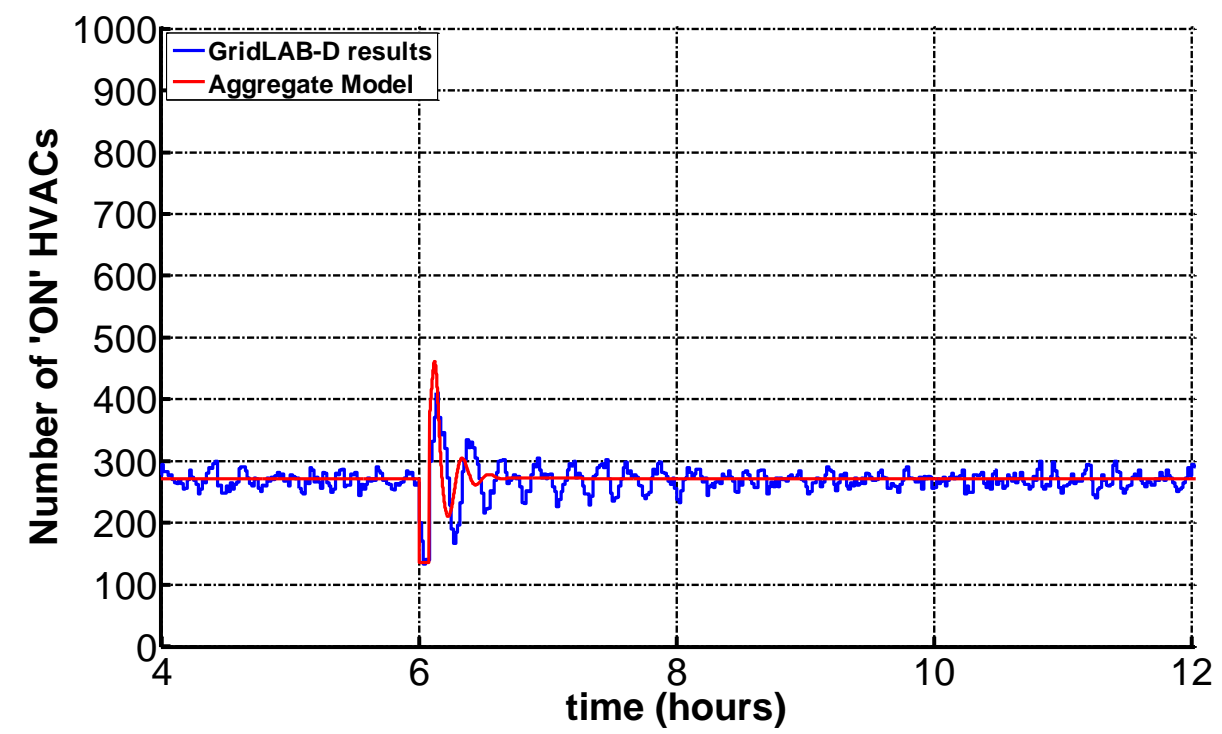

Figure 43 Outdoor temperature of $85^{\circ} \mathrm{F}$, lockout times set to two minutes, and a $50 \%$ reduction in load that releases back to normal operation after 5 minutes 


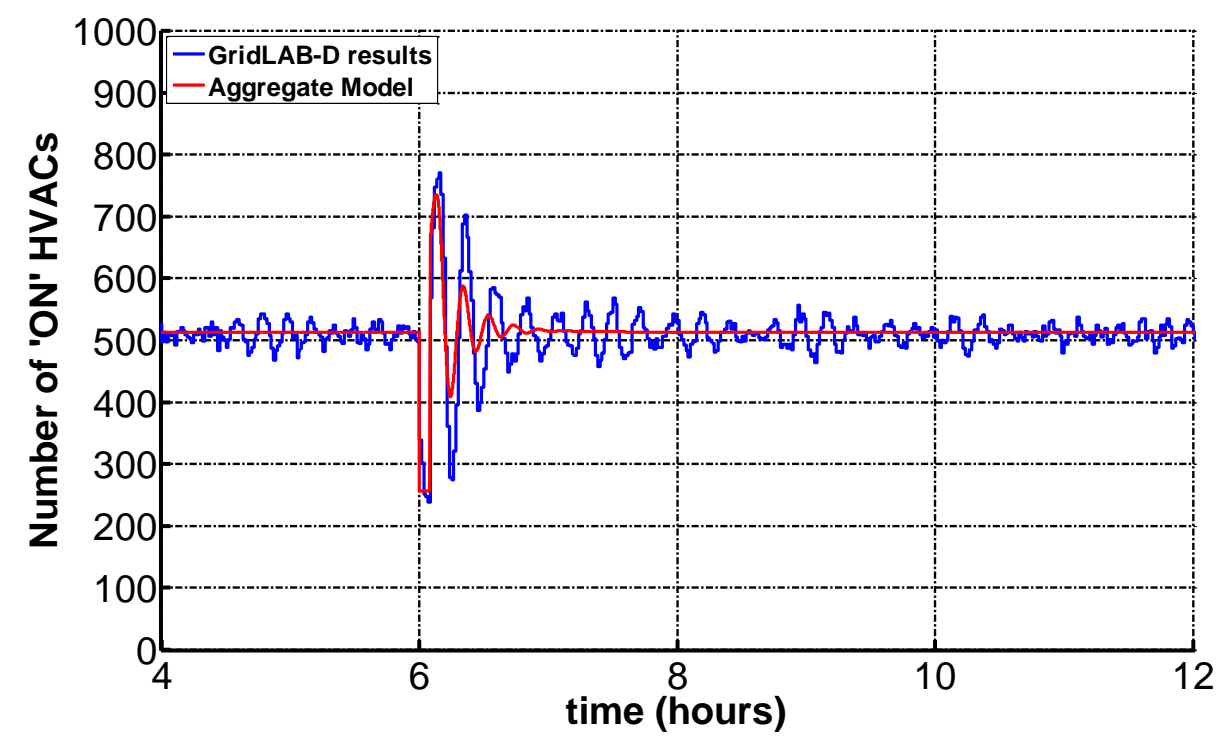

Figure 44 Outdoor temperature of $100^{\circ} \mathrm{F}$, lockout times set to two minutes, and a $50 \%$ reduction in load that releases back to normal operation after 5 minutes

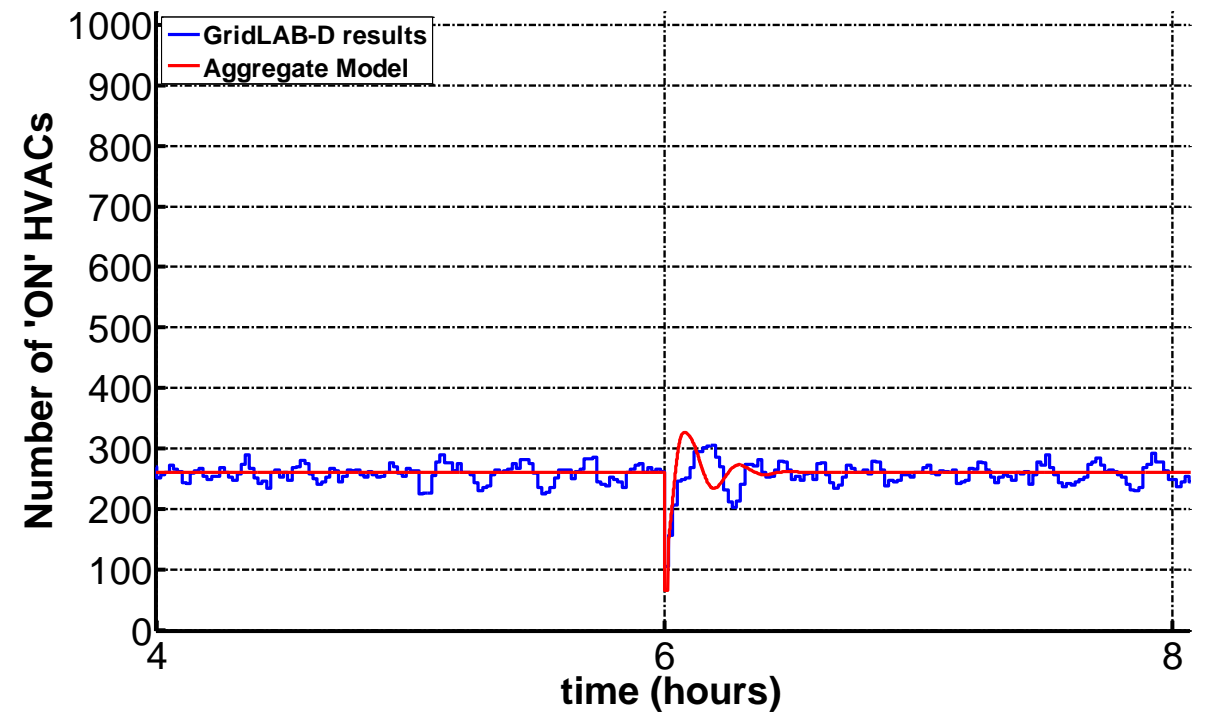

Figure 45 Outdoor temperature of $85^{\circ} \mathrm{F}$, lockout times set to (near) zero, and a $75 \%$ reduction in load that releases back to normal operation after 30 seconds 


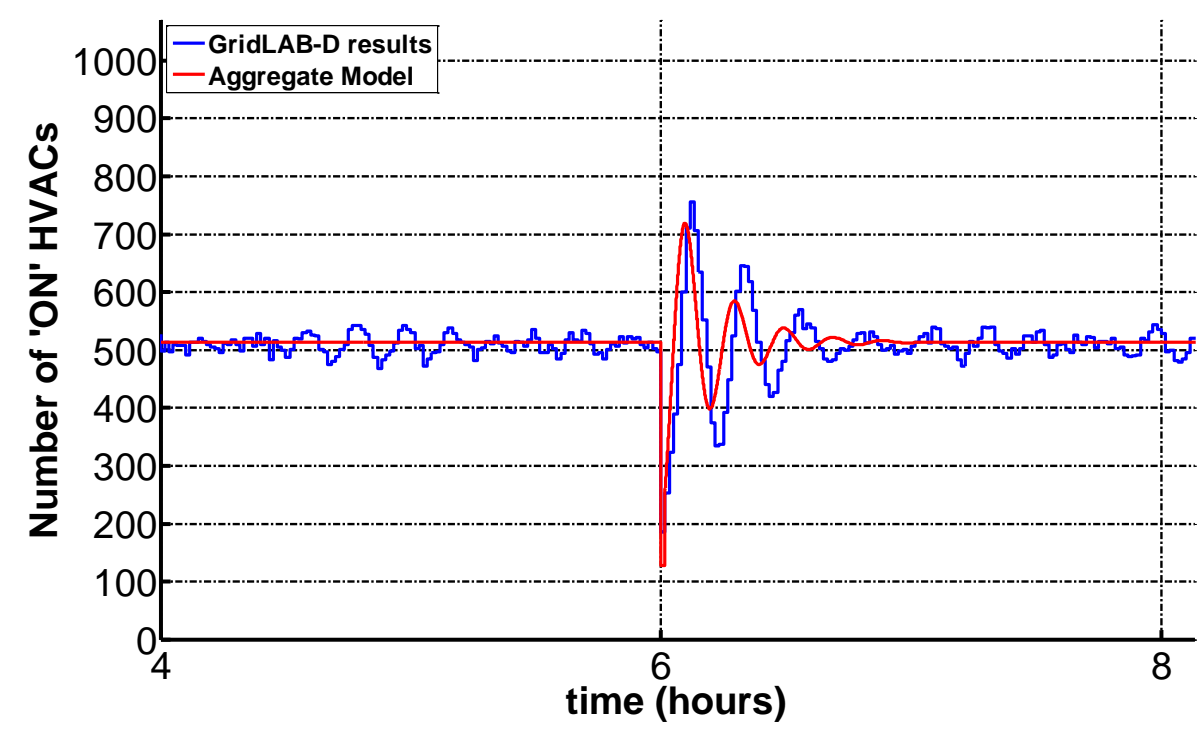

Figure 46 Outdoor temperature of $100^{\circ} \mathrm{F}$, lockout times set to (near) zero, and a $75 \%$ reduction in load that releases back to normal operation after 30 seconds

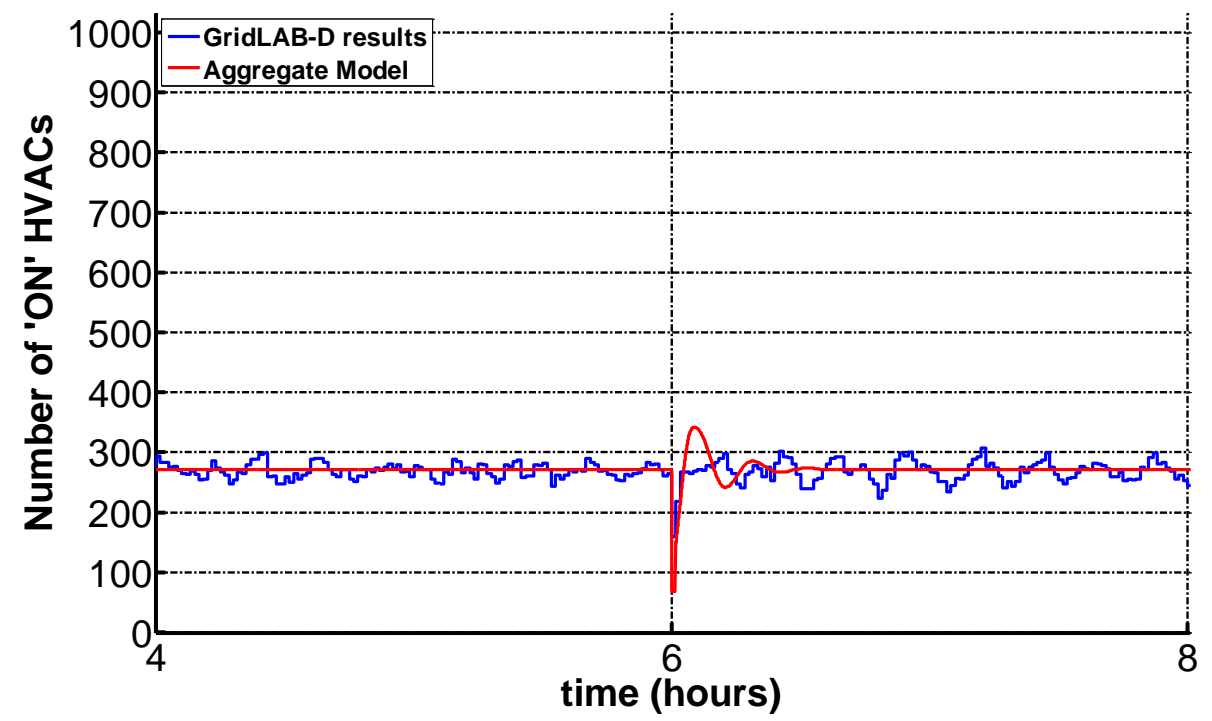

Figure 47 Outdoor temperature of $85^{\circ} \mathrm{F}$, lockout times set to two minutes, and a $75 \%$ reduction in load that releases back to normal operation after 30 seconds 


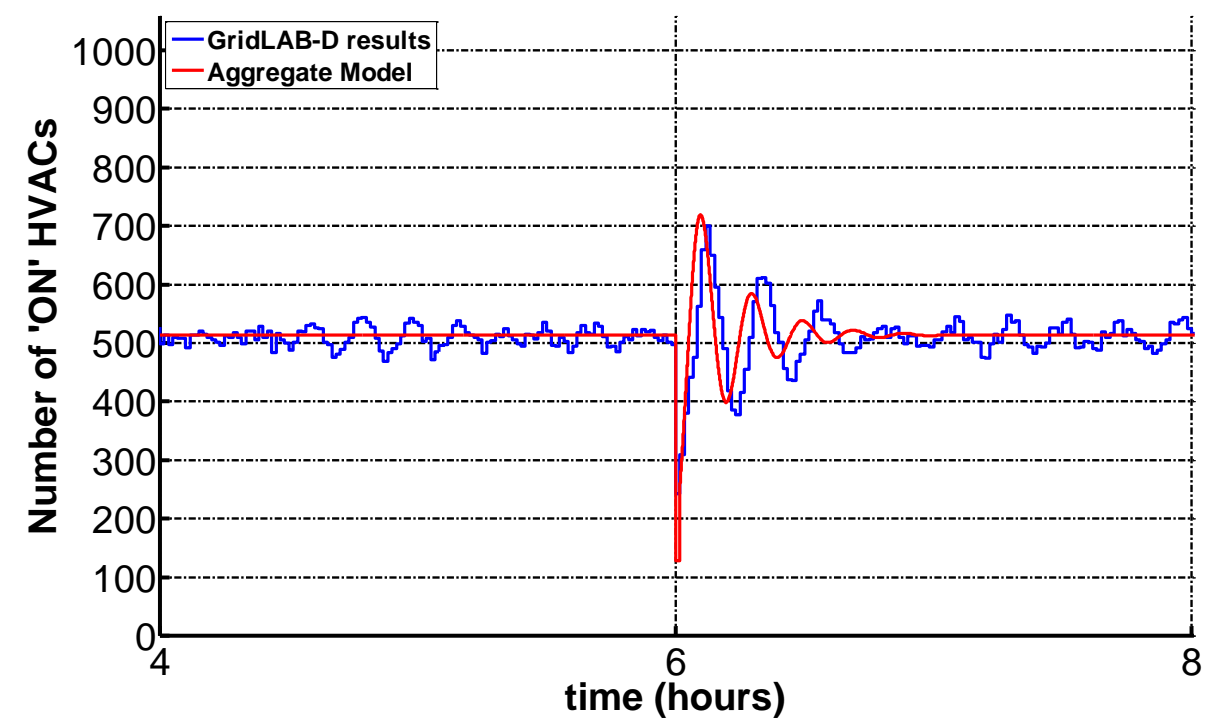

Figure 48 Outdoor temperature of $100^{\circ} \mathrm{F}$, lockout times set to two minutes, and a $75 \%$ reduction in load that releases back to normal operation after 30 seconds

\section{A.3.2 Closed-loop control: Frequency regulation signal}

These tests were designed to represent control for use with a frequency regulation signal that the devices could respond to by reducing power demand during low frequency events and increase demand during high frequency events. This requires very short term, but minimal adjustments in total power demand. The frequency signal was extracted from PJM historical data [20] and converted into a power reference signal. The devices were commanded to respond for 40 minutes (times 1.5 to 2.2 hours) then released back to normal operation (note, in the figures the reference signal is shown prior to time 1.5 and after time 2.2 , however the devices only responded to the signal within the designated time period). The individual level device controls are described in Section 2.4.2.1. The model is adaptive and corrects itself at 10second time steps by comparing its assumed power demand against the "actual" power demand of GridLAB-D and adjusts its internal states accordingly. In these cases, whether the thermostat lockout was used or not, the model and control signal were able to accurately control the individual devices to the reference signal, with only a minor difference at the highest load period due to the reference signal saturating the devices. 


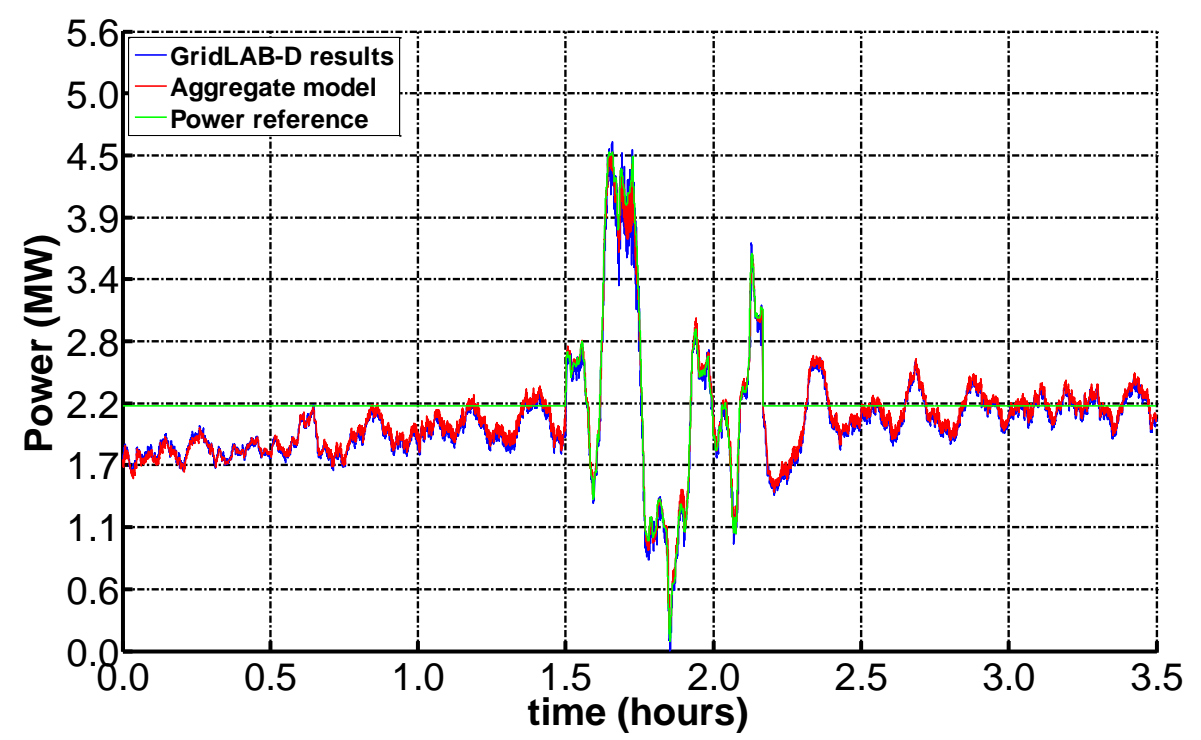

Figure 49 Outdoor temperature of $85^{\circ} \mathrm{F}$ and average setpoint of $75^{\circ} \mathrm{F}$, lockout times set (near) zero

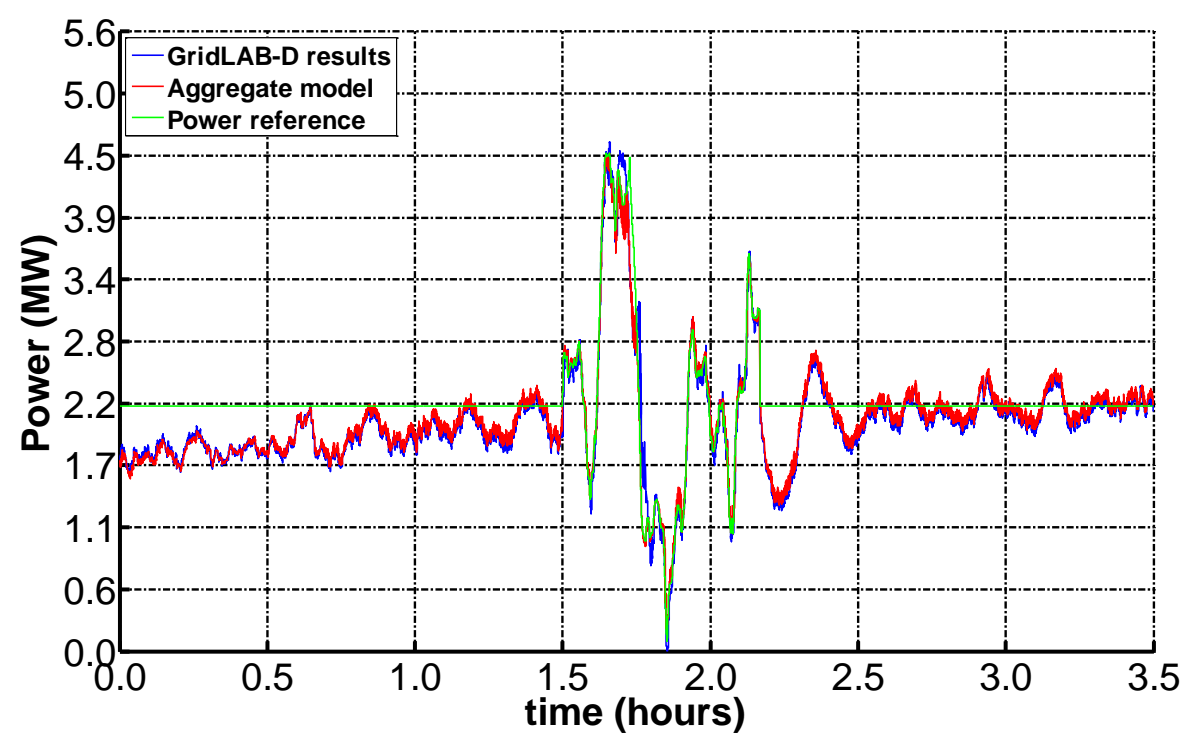

Figure 50 Outdoor temperature of $85^{\circ} \mathrm{F}$ and average setpoint of $75^{\circ} \mathrm{F}$, lockout times set to two minutes

\section{A.3.3 Closed-loop control: Reference signal}

Finally, to determine the extent to which the aggregate model can be used to directly control a diverse population, a variety of additional reference signals were created and tracked using the power demand from the GridLAB-D models as a feedback mechanism into the aggregate model. This was used to account for the effects of a "real" system which contains a number of unknowns and losses throughout the system. The model is adaptive and corrects itself at each 10-second time step by comparing its assumed power demand against the "actual" power demand of GridLAB-D and adjusts its internal states 
accordingly. The individual device level controls are the same used in the previous section. The following figures show the results from these tests. In general, the model and GridLAB-D were able to sufficiently track the reference signal. Of note are the small oscillations seen in the aggregate model as it attempts to correct to the GridLAB-D power demand, then drifts back towards its predicted state, then again corrects to the GridLAB-D power demand after ten seconds. The oscillations are relatively small, but noticeable, and are caused by the fact that the aggregate model is updated every second, while the correction step only occurs every ten seconds. Also note that there is almost no difference between the "lossy" and "lossless" system response, as the closed loop control corrects for the error within the model. In Figure 51, Figure 52, and Figure 53, the load oscillates after the signal is released. This is due to the artificial synchronization of the loads caused by the control signal. In actual applications, this rebound and oscillation could be reduced by controlling the devices not only during the control period but immediately following the demand response event.

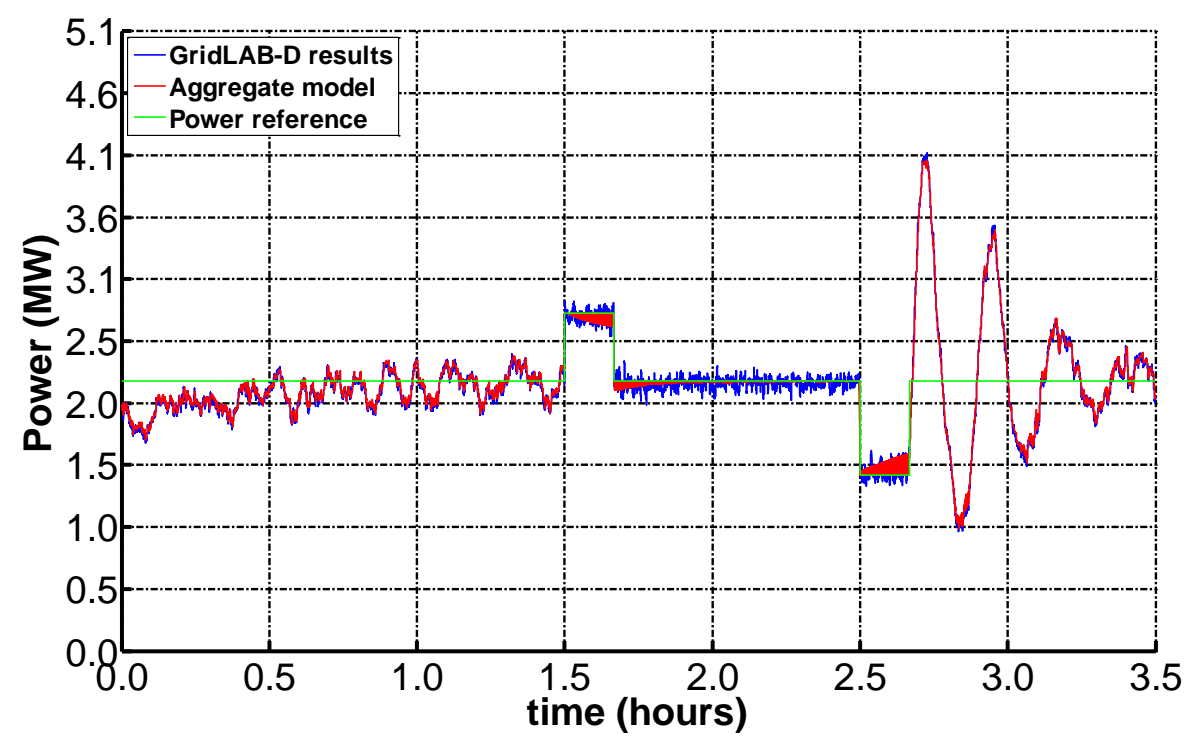

Figure 51 Outdoor temperature of $85^{\circ} \mathrm{F}$, lockout times set to two minutes, a "lossless" system, with a $25 \%$ increase in load for 10 minutes, returning to normal for 50 minutes, then a $35 \%$ decrease in load for 10 minutes, and then returning to normal. 


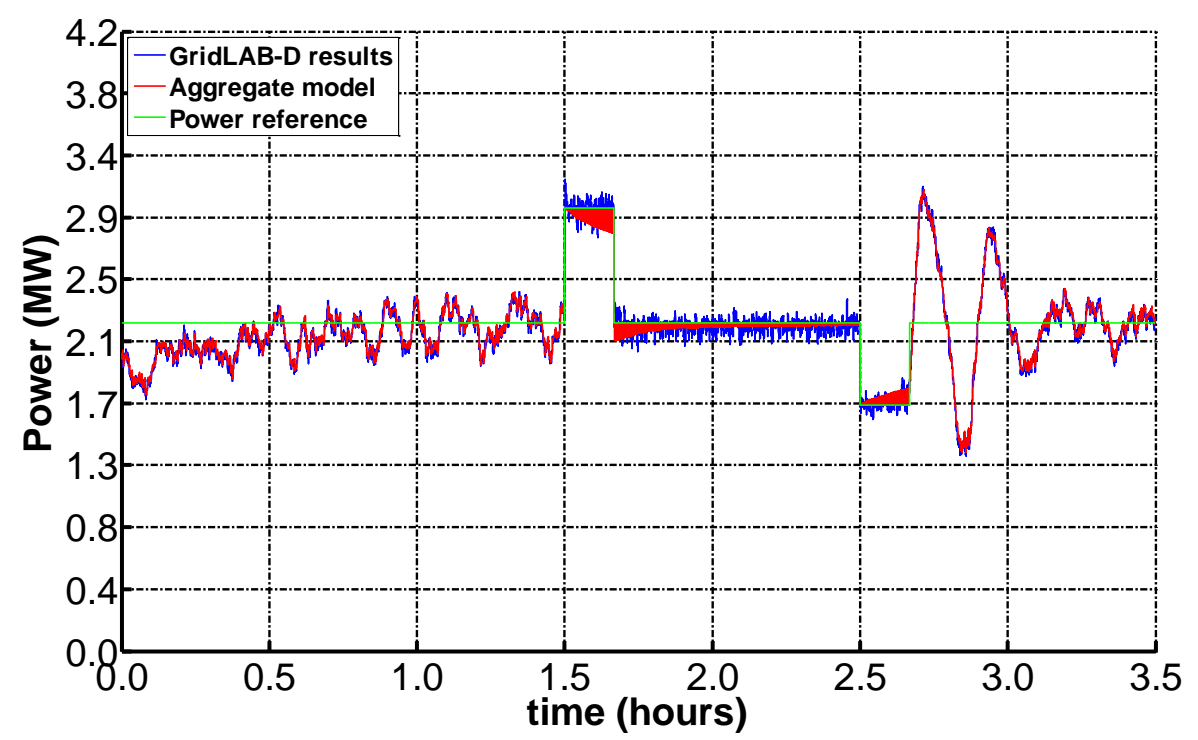

Figure 52 Outdoor temperature of $85^{\circ} \mathrm{F}$, lockout times set to two minutes, a "lossy" system, with a $35 \%$ increase in load for 10 minutes, returning to normal for 50 minutes, then a $25 \%$ decrease in load for 10 minutes, and then returning to normal.

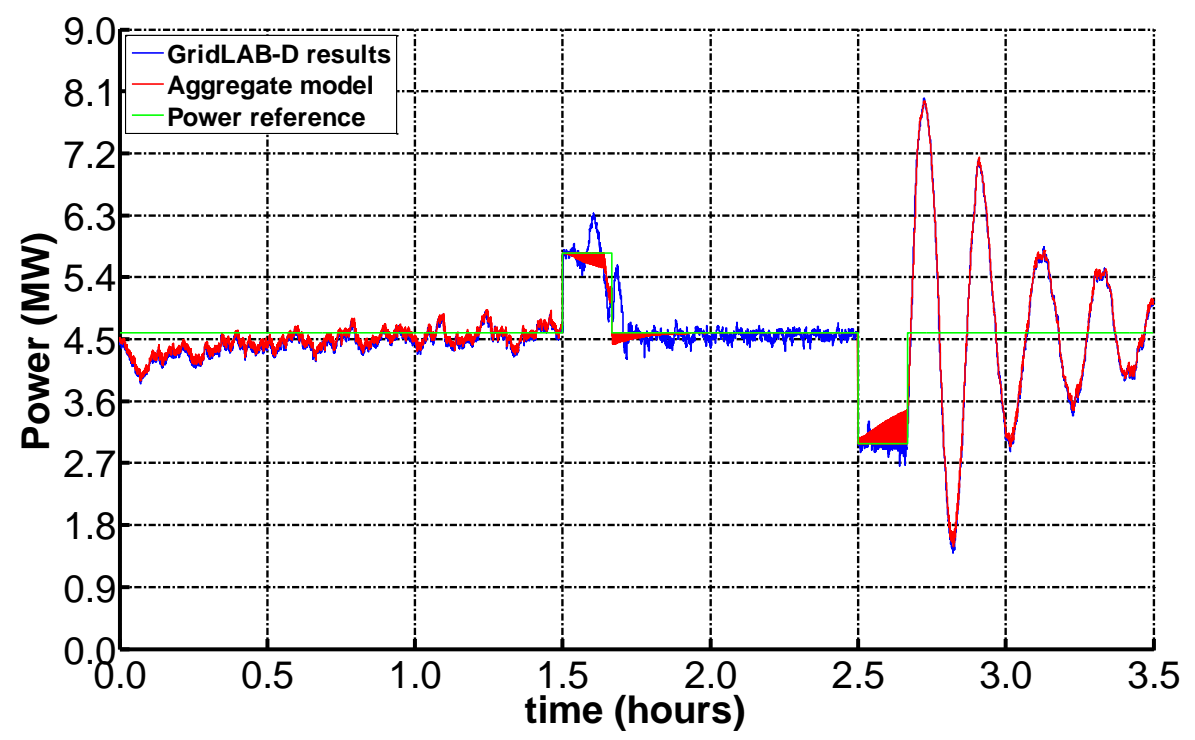

Figure 53 Outdoor temperature of $100^{\circ} \mathrm{F}$, lockout times set to two minutes, a "lossless" system, with a $25 \%$ increase in load for 10 minutes, returning to normal for 50 minutes, then a $35 \%$ decrease in load for 10 minutes, and then returning to normal. 


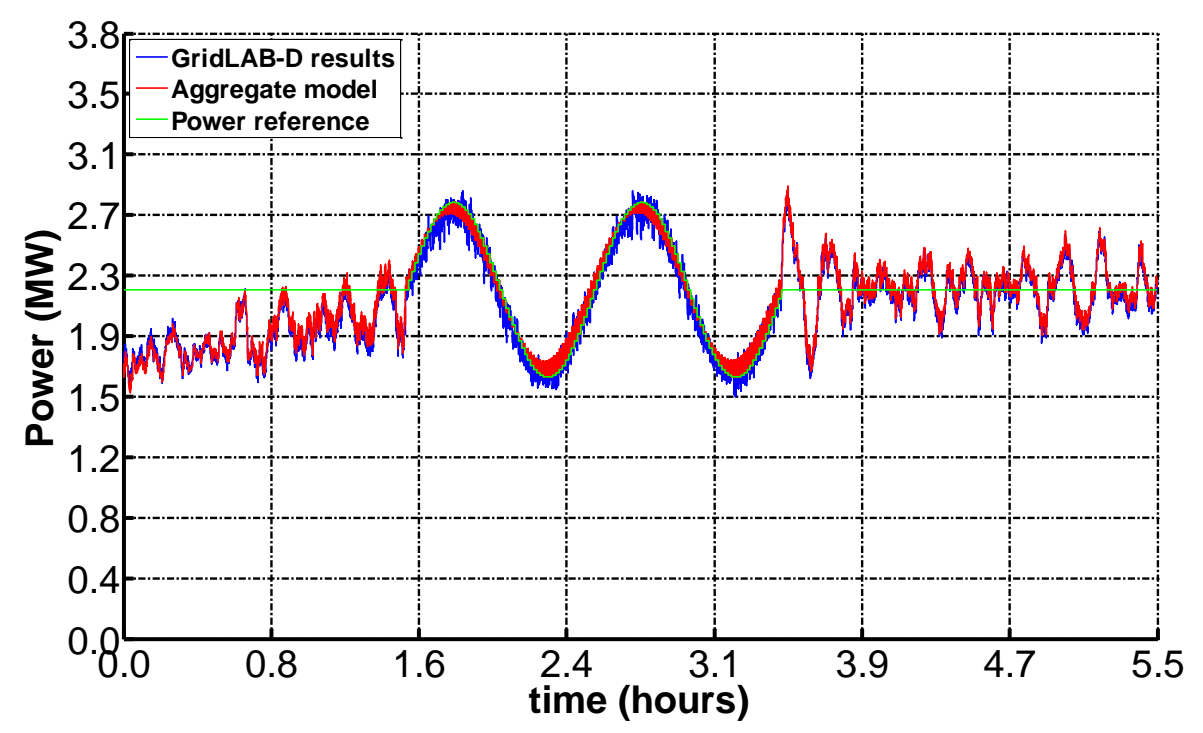

Figure 54 Outdoor temperature of $85^{\circ} \mathrm{F}$, lockout times set to two minutes, a "lossy" system, with a control signal that is a sinusoidal signal of power updated every minute with a maximum swing of $25 \%$ of normal power demand with a frequency of 1 hour

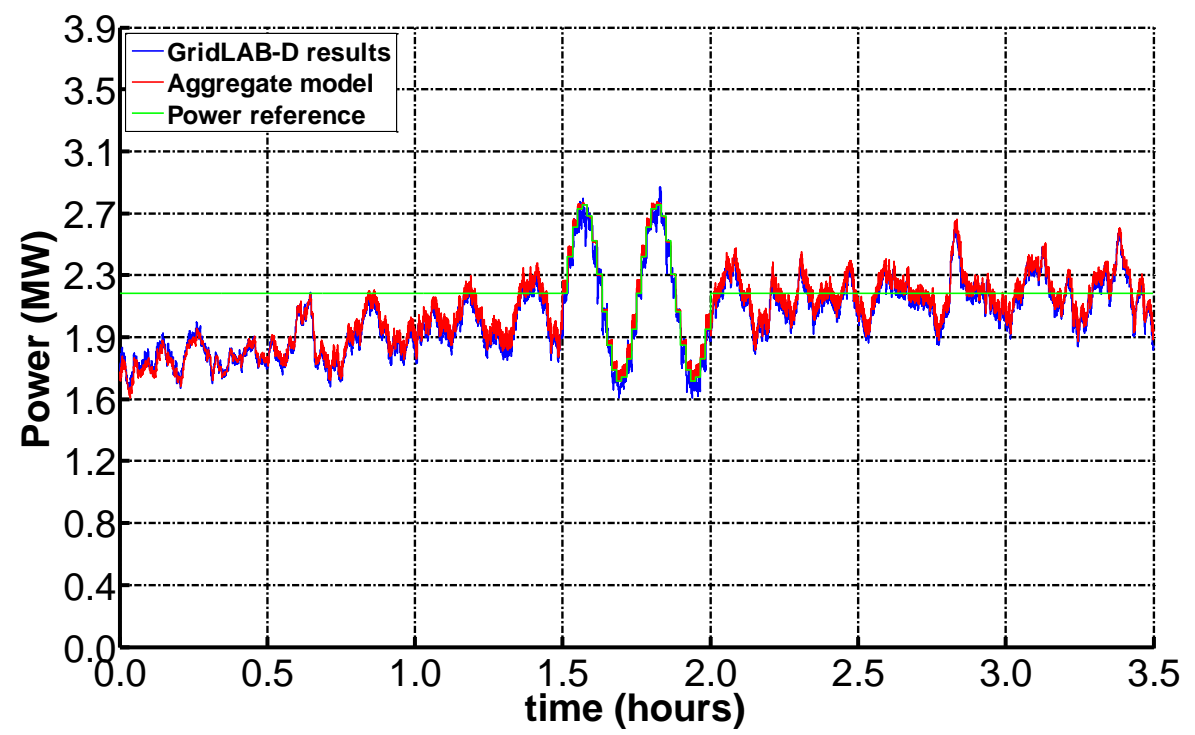

Figure 55 Outdoor temperature of $85^{\circ} \mathrm{F}$, lockout times set to two minutes, a "lossy" system, with a control signal that is a sinusoidal signal of power updated every minute with a maximum swing of $25 \%$ of normal power demand with a frequency of 15 minutes 


\section{Distribution}

1 Dan Ton

Department of Energy, OE

1000 Independence Ave., SW

Routing OE-10

Washington, DC 20585
8 Local Distribution

Pacific Northwest National Laboratory

Karanjit Kalsi

(PDF)

Jason Fuller

(PDF)

Francis Tuffner (PDF)

Jianming Lian (PDF)

Laurentiu Marinovici (PDF)

Andrew Fisher (PDF)

Forrest Chassin (PDF)

Matt Hauer (PDF)

Robert Pratt (PDF)

1 Wei Zhang

404 Dreese Labs,

2015 Neil Ave.,

Columbus, $\mathrm{OH}, 43210$

(PDF copy) 


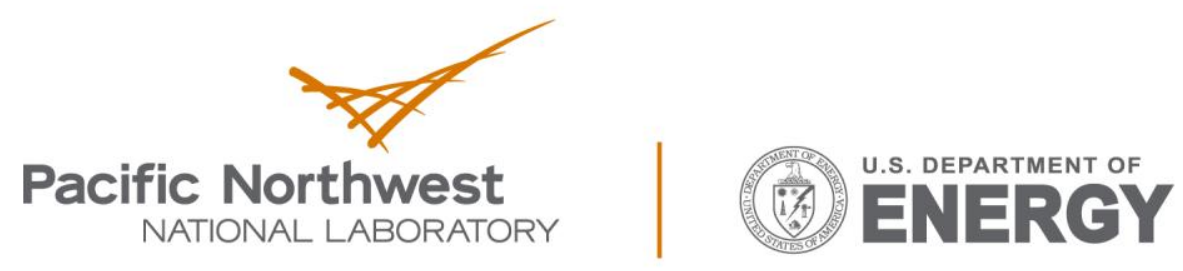

Proudly Operated by Battelle Since 1965

902 Battelle Boulevard

P.O. Box 999

Richland, WA 99352

1-888-375-PNNL (7665)

www.pnnl.gov 